\title{
Partial Regularity for Holonomic Minimisers of Quasiconvex Functionals
}

\author{
Christopher P. Hopper \\ Communicated by C. DE LELLIS
}

\begin{abstract}
We prove partial regularity for local minimisers of certain strictly quasiconvex integral functionals, over a class of Sobolev mappings into a compact Riemannian manifold, to which such mappings are said to be holonomically constrained. Our approach uses the lifting of Sobolev mappings to the universal covering space, the connectedness of the covering space, an application of Ekeland's variational principle and a certain tangential $\mathbb{A}$-harmonic approximation lemma obtained directly via a Lipschitz approximation argument. This allows regularity to be established directly on the level of the gradient. Several applications to variational problems in condensed matter physics with broken symmetries are also discussed, in particular those concerning the superfluidity of liquid helium-3 and nematic liquid crystals.
\end{abstract}

\section{Introduction and Statement of Main Result}

Let $\mathcal{M}$ be a compact submanifold of $\mathbb{R}^{N}$ without boundary and $\Omega \subset \mathbb{R}^{n}$ be a Lipschitz domain. We define the Sobolev space of mappings into $\mathcal{M}$ by

$$
W^{s, p}(\Omega, \mathcal{M})=\left\{u \in W^{s, p}\left(\Omega, \mathbb{R}^{N}\right): u(x) \in \mathcal{M} \text { almost everywhere } x \in \Omega\right\}
$$

for $s \geqq 1$ and $1 \leqq p \leqq \infty$. Note that the definition is independent of isometric embedding (cf. [50, Lem. 1.4.3]) and the set $W^{s, p}(\Omega, \mathcal{M})$ inherits strong and weak topologies from $W^{s, p}\left(\Omega, \mathbb{R}^{N}\right)$. The local space $W_{\text {loc }}^{s, p}(\Omega, \mathcal{M})$ consist of maps belonging to $W^{s, p}\left(\Omega^{\prime}, \mathcal{M}\right)$ for all $\Omega^{\prime} \subset \subset \Omega$ (that is for all $\Omega^{\prime}$ compactly embedded in $\Omega$ ).

Given an integral functional of the form

$$
\mathfrak{F}(v ; \Omega)=\int_{\Omega} f(x, v, D v) \mathrm{d} x,
$$

where $v: \Omega \subset \mathbb{R}^{n} \rightarrow \mathbb{R}^{N}$ with $n, N \geqq 2$, we say: 
Definition 1.1. A map $u \in W_{\text {loc }}^{1, p}(\Omega, \mathcal{M})$ is an holonomically constrained local minimiser of $\mathfrak{F}$, or simply an holonomic $\mathfrak{F}$-minimiser, if

$$
\mathfrak{F}\left(u ; \Omega^{\prime}\right) \leqq \mathfrak{F}\left(v ; \Omega^{\prime}\right)
$$

for all $v \in\left(u+W_{0}^{1, p}\left(\Omega^{\prime}, \mathbb{R}^{N}\right)\right) \cap W^{1, p}\left(\Omega^{\prime}, \mathcal{M}\right)$ and all open $\Omega^{\prime} \subset \subset \Omega{ }^{1}$

Note that the competitor $v$ to the minimiser $u$ must fulfil the manifold constrained condition as well as agreeing with the trace of $u$ on the boundary-in the sense that $v-u \in W_{0}^{1, p}\left(\Omega, \mathbb{R}^{N}\right)$, where $W_{0}^{1, p}\left(\Omega, \mathbb{R}^{N}\right)$ is the closure of the smooth compactly supported functions $C_{c}^{\infty}\left(\Omega, \mathbb{R}^{N}\right)$ in the $\|\cdot\|_{W^{1, p}}$-norm.

In this paper we shall consider an integrand $f: \Omega \times \mathbb{R}^{N} \times \mathbb{R}^{N n} \rightarrow \mathbb{R}$, for $p \geqq 2$, which satisfies the following structural assumptions, namely:

(h0) The function $\xi \mapsto f(x, u, \xi)$ is $C^{2}$ and $(x, u, \xi) \mapsto D_{\xi \xi}^{2} f(x, u, \xi)$ is jointly continuous.

(h1) $f$ satisfies the $p$-growth condition

$$
|f(x, u, \xi)| \leqq \Gamma\left(1+|\xi|^{p}\right)
$$

for some constant $\Gamma>0$ and all $(x, u, \xi)$.

(h2) $f$ satisfies Morrey's quasiconvexity condition in the following strong form

$$
\begin{aligned}
\gamma \int_{\Omega^{\prime}}\left(1+|\xi|^{2}+|D \phi(y)|^{2}\right)^{\frac{p-2}{2}}|D \phi(y)|^{2} \mathrm{~d} y \leqq & \int_{\Omega^{\prime}}(f(x, u, \xi+D \phi(y)) \\
& -f(x, u, \xi)) \mathrm{d} y
\end{aligned}
$$

for some constant $\gamma>0$, every $(x, u, \xi)$, every bounded open $\Omega^{\prime} \subset \mathbb{R}^{n}$ and every $\phi \in C_{c}^{\infty}\left(\Omega^{\prime}, \mathbb{R}^{N}\right)$.

(h3) $f$ satisfies

$$
|f(x, u, \xi)-f(y, v, \xi)| \leqq \Gamma \omega_{\mu}(|x-y|+|u-v|)\left(1+|\xi|^{p}\right)
$$

where $\omega_{\mu}(t)=\min \left(1, t^{\mu}\right)$ for a given $0<\mu \leqq 1$.

(h4) There exists a continuous $\psi: \mathbb{R}^{N n} \rightarrow \mathbb{R}$ satisfying

$$
f(x, u, \xi) \geqq \psi(\xi) \text { and } \int_{\Omega} \psi(D \varphi) \geqq \int_{\Omega} \psi(0)+\gamma|D \varphi|^{p}
$$

for every $\varphi \in C_{c}^{\infty}\left(\Omega, \mathbb{R}^{N}\right)$.

${ }^{1}$ Following the terminology introduced by HeRTz [51, p. 91], subsidiary conditions of this type are referred to as holonomic constraints (from ö $\lambda \circ$, , holos - whole, entire, complete in all its parts; vó $\mu$ os, nomos - law 〈taken to mean 'integrable' via the Latin integer — complete, whole, entire, intact $\rangle$ ). 
We remark that (h4) is obviously satisfied if $f(x, u, \xi) \geqq|\xi|^{p}$ but also allows for integrands with varying sign. It is easy to show that the growth condition (h1) together with the quasiconvexity of (h2) is enough (in fact $f$ need only to be rankone convex) for the derivative $D_{\xi} f$ to satisfy the growth condition

$$
\left|D_{\xi} f(x, u, \xi)\right| \leqq c\left(1+|\xi|^{p-1}\right)
$$

and

$$
|f(x, u, \xi+\eta)-f(x, u, \xi)| \leqq c\left(1+|\xi|^{p-1}+|\eta|^{p-1}\right)|\eta| .
$$

As remarked in [3], there is no growth condition placed on the second order derivatives of $\xi \mapsto f(x, u, \xi)$. We merely have, for any given $\Omega^{\prime} \subset \subset \Omega$ and any given $\Lambda>0$, the existence of a finite $K_{\Lambda}$ such that

$$
\sup _{x \in \bar{\Omega}^{\prime}|u|,|\xi| \leqq \Lambda} \sup _{\xi \xi}\left|D_{\xi \xi}^{2} f(x, u, \xi)\right| \leqq K_{\Lambda} .
$$

There are also no global uniform continuity assumptions imposed on $D_{\xi \xi}^{2} f(x, u, \xi)$ either. One only has $(u, \xi) \mapsto D_{\xi \xi}^{2} f(x, u, \xi)$ uniformly continuous on compact subsets. In fact, associated to $\Lambda>0$, a min-max construction implies the existence of a non-decreasing concave modulus of continuity $\omega:[0,+\infty) \rightarrow[0,1]$, with $\lim _{t \searrow 0} \omega(t)=0$ and $\omega(t)=1$ for all $t \geqq 1$, such that

$$
\left|D_{\xi \xi}^{2} f\left(x, u_{1}, \xi_{1}\right)-D_{\xi \xi}^{2} f\left(x, u_{2}, \xi_{2}\right)\right| \leqq c \omega\left(\left|u_{1}-u_{2}\right|+\left|\xi_{1}-\xi_{2}\right|\right)
$$

for any $\left|u_{i}\right|,\left|\xi_{i}\right| \leqq \Lambda+1$ and $x \in \bar{\Omega}^{\prime}$.

In considering the above geometric and analytic aspects, we seek to prove the following main result:

Theorem 1.2. Let $\mathcal{M}$ be a connected compact $C^{3}$-Riemannian manifold without boundary and $\pi: \widetilde{\mathcal{M}} \rightarrow \mathcal{M}$ be its universal covering. Suppose the fundamental group $\pi_{1}(\mathcal{M})$ is finite, $\widetilde{\mathcal{M}}$ is $\ell$-connected for some integer $1 \leqq \ell \leqq n-2$, and the integrand $f$ satisfies $(h 0),(h 1),(h 2),(h 3)$ and $(h 4)$ for $2 \leqq p<\ell+2$. Then for any holonomic $\mathfrak{F}$-minimiser $u \in W_{\text {loc }}^{1, p}(\Omega, \mathcal{M})$ there is a relatively closed subset Sing $u \subset \Omega$ of Lebesgue measure zero such that

$$
u \in C_{\mathrm{loc}}^{1, \beta}(\Omega \backslash \operatorname{Sing} u, \mathcal{M})
$$

for some $0<\beta<1$.

Note that a topological space $X$ is said to be $\ell$-connected if its first $\ell$ homotopy groups vanish identically, that is

$$
\pi_{0}(X)=\pi_{1}(X)=\cdots=\pi_{\ell-1}(X)=\pi_{\ell}(X)=0 .
$$

A non-empty space $X$ is 0 -connected if and only if it is pathwise connected. A space $X$ is 1-connected if and only if it is simply connected.

Recall also that (1) every connected manifold admits a universal covering by a simply connected manifold; (2) a connected compact manifold has a compact universal cover if and only if the fundamental group of the base space is finite; 
and that (3), the homotopy groups of the covering space of order higher than 2 are equal to the homotopy groups of the base space (cf. $[49,52])$. In our proof, the compactness and connectedness of covering space is needed in Section 4 for the construction of suitable comparison maps.

Example 1.3. If $k \geqq 2$ is an integer we have:

\begin{tabular}{l||ll|l|l|l}
$\mathcal{M}$ & $\pi_{0}$ & $\pi_{1}$ & $\mathrm{UC}$ & $\ell$ & \\
\hline$S^{k}$ & 0 & 0 & $S^{k}$ & $k-1$ & $k$-sphere \\
$\mathbb{R} P^{k}$ & 0 & $\mathbb{Z}_{2}$ & $S^{k}$ & $k-1$ & Real projective space \\
$\mathrm{SO}(k+1)$ & 0 & $\mathbb{Z}_{2}$ & $\operatorname{Spin}(k+1)$ & 2 & Special orthogonal group \\
$U(k)$ & 0 & $\mathbb{Z}$ & $\mathbb{R} \times S U(k)$ & 2 & Unitary group
\end{tabular}

When the manifold $\mathcal{M}$ is a connected compact Lie group $G$, and $\mathfrak{g}$ is the Lie algebra of $G$, the simply connected covering group splits (cf. [57, Theor. 6.6]) into $\widetilde{\mathcal{M}}=\mathbb{R}^{\lambda} \times G_{1} \times \cdots \times G_{\nu}$, where $\lambda=\operatorname{dim}_{\mathbb{R}} \mathfrak{c}$ of the centre $\mathfrak{c}$ of $\mathfrak{g}$. As the simply connected simple compact Lie groups $G_{\mu}$ are 2 -connected we have $2 \leqq p<4$. $^{2}$

Remark 1.4. From the above theorem and its proof one would expect the Hausdorff dimension of Sing $u$ to be strictly smaller than $n$ when $u$ is additionally assumed to be Lipschitz continuous (cf. [59] for unconstrained Lipschizian minimisers of strictly quasiconvex integral functionals with $p$-growth).

The canonical holonomically constrained variational problem is that of harmonic maps between Riemannian manifolds which minimise the Dirichlet energy. The question of regularity for minimisers of the Dirichlet energy in this constrained context was first obtained by MORREY [70] in connection with his analysis of Plateau's problem. By working with absolutely continuous representatives Morrey showed minimisers to be locally Hölder continuous when $n=2$. The harmonic map regularity result was extended to the case $n>2$ by the seminal work of Schoen and Uhlenbeck [79]. By working with the class (1) they showed energy minimising maps with a compact image to be locally smooth away from a closed (singular) subset that is discrete if $n=3$ and has Hausdorff dimension at most $n-3$ when $n \geqq 4$. A refined treatment of this partial regularity result, as well as further analysis on the rectifiability of the singular set, can be found in the book of SIMON [78]. Similar results for the case in which the image lies in a single coordinate chart were obtained by GIAQUiNTA and GiUsti $[40,41]$ (and by $[54,58]$ when the image of the map is contained in a convex ball of $\mathcal{M}$ ). In the $p$-harmonic case, HARDT and LIN [56] showed $p$-energy minimisers, for $p>1$, to be $C_{\text {loc }}^{1, \alpha}$ for some $0<\alpha<1$ except possibly on a closed set of Hausdorff dimension at most $n-\lfloor p\rfloor-1$. Further partial regularity results in the $p$-harmonic case for maps into a compact target manifold can be found in $[28,64]$. The question of minimising in an appropriate homotopy class has also been studied by White $[83,84]$.

Partial regularity results for a more general class of convex functionals have been obtained by LUCKHAUs [63] with appropriately well-behaved blow-ups. In this

${ }^{2}$ Note that $\pi_{2}(G)=0$ for any connected compact Lie group $G$ (cf. [14, p. 225]) and that $\pi_{3}(G)=\mathbb{Z}$ for any simply connected simple compact Lie group $G$ (cf. [8]). 
direction, results for convex functionals with $p$-growth can also be found in $[53,56]$. In particular, [56] obtained reverse Hölder inequalities under the assumption $\mathcal{M}$ is $(\lfloor p\rfloor-1)$-connected via a retraction of $\mathbb{R}^{N} \backslash X$ onto $\mathcal{M}$ for some polyhedron $X$ in $\mathbb{R}^{N} \backslash \mathcal{M}$. This kind of retraction was previously used in (the domain) by [83] for the existence of finite energy extensions of Lipschitz maps. In fact the idea of using such a continuous retraction with analytic estimates goes back to the proof in [37] of isoperimetric inequalities.

To prove the existence of (unconstrained) minimisers of general integral functionals subject to given boundary data, MoRREY [71] introduced a notion of quasiconvexity which isolated a property of the integrand that, under appropriate growth conditions, is necessary and sufficient for the weak sequential lower semicontinuity of the functional. A result of ACERBI and FUSCO [2] shows that a non-negative Carathéodory integrand with $p$-growth is weakly sequentially lower semicontinuous on the $W^{1, p}$-Sobolev space if and only if the integrand is quasiconvex. Regularity considerations for such quasiconvex functionals in the unconstrained case were initiated by Evans [32], who showed a slightly strengthened version of quasiconvexity (that is the so-called uniformly strict quasiconvex condition) forces partial regularity. That is to say, if $u$ minimises a functional of the form $\int_{\Omega} f(D u) \mathrm{d} x$, where $f$ is a strictly quasiconvex $C^{2}$-function with $p$-growth, $p \geqq 2$, that satisfies an additional bound on the second derivatives $D^{2} f$, then the gradient $D u$ is locally Hölder continuous outside a set of Lebesgue measure zero. This result was subsequently generalised to integrals with $x$ - and $u$-dependences in $[39,46]$ and the bound for $D^{2} f$ was removed in [3]. An extension to the subquadratic case $1<p<2$ has also been obtained in [17]. A partial regularity result for a class of strong local minimisers of quasiconvex functionals can be found in [60]. Further details on other aspects in the unconstrained case [including boundary regularity, degenerate cases, conditions for everywhere regularity, $\omega$-minima, $(p, q)$-growth conditions and counterexamples to full regularity] can be found in the survey article [69].

In order to obtain partial regularity, a linearisation strategy is often used to transfer the 'good' regularity properties of the linearised elliptic system to the nonlinear problem. The technique of harmonic-type approximations, in particular, has proved both a popular and elemental way of obtaining partial regularity for solutions to non-linear elliptic systems and minimisers of certain integral functionals (cf. [29]). The underlying premise of this method goes back to ideas introduced by De Giorgi [22] in his treatment of minimal surfaces. Specifically, he establishes 'some properties of regular surfaces approximating minimal surfaces; these properties will be obtained by a comparison argument with the graph of suitable harmonic functions' [23, p. 244]. In the context of regularity for elliptic systems the technique was utilised by SIMON [78, §1.6] to provide a neat and elementary proof of partial regularity. For quasiconvex integrals this method was initiated in [24]. The proof of harmonic-type approximation results is typically achieved through an indirect compactness argument, nonetheless a version using 'a direct approach based on the Lipschitz truncation technique which requires no contradiction argument' has recently been proved in $[27,30]$. 
For minimisers of quasiconvex functionals in the manifold constrained case, the only know result to date (aside from the results in $[53,56,63]$ for holonomic minimiser of convex functionals) is that of the EvANS and GARIEPY paper [34]. By appealing to the blow-up technique introduced in [33], Evans and Gariepy consider a functional of the form $\int_{\Omega} f(D u) \mathrm{d} x$ under the structural hypothesis that $f$ is a strictly quasiconvex $C^{2}$-function for $p=2$ which satisfies an additional bound on the second derivatives $D^{2} f$. Comparison maps satisfying the non-linear manifold constraints in the indirect blow-up proof are obtained from a particular extension lemma of LuCKHAUs (cf. [63,64] and also [48,55,78]), however this construct only works in the blow-up case due to the order in which limits are taken (cf. [34, p. 87]).

Remark 1.5. (Idea of proof and applicability of Luckhaus' lemma) Given $f_{1} \in$ $W^{1, p}(\mathcal{M}, \mathcal{N})$ and $f_{2} \in W^{1, p}(\mathcal{M}, \mathcal{O})$ for $p \geqq 2$, where $\mathcal{M}^{m} \subset \mathbb{R}^{\ell}$ is a Riemannian manifold without boundary and $\mathcal{N} \subset \mathbb{R}^{k}$ is a closed subset of positive reach with a neighbourhood $\mathcal{O}$, the so-called Luckhaus lemma seeks to construct a function $f \in$ $W^{1, p}\left(\mathcal{M} \times(0, \lambda), \mathbb{R}^{k}\right)$ for $0<\lambda<1$, with $f(\cdot, 0)=f_{1}$ and $f(\cdot, \lambda)=f_{2}$, such that one has $L^{p}$-control of the $\operatorname{dist}(f, \mathcal{N})$ - so that it can be made to be sufficiently small. Normally in the unconstrained setting $f$ would be constructed simply as a convex combination of $f_{1}$ and $f_{2}$, however to gain the extra control on $\operatorname{dist}(f, \mathcal{N})$ a triangulation of the domain $\mathcal{M}$ is employed so as to restrict the Sobolev mappings to lower-dimensional skeletons. For such skeletons of dimension $d \leqq\lfloor p-1\rfloor$ one can use a convex combination and a Morrey-type embedding to construct and gain the desired pointwise control, that is if $u, v \in W^{1,1}(B)$ on a ball $B \subset \mathbb{R}^{d}$ of radius $r$,

$$
\begin{aligned}
|u(x)-v(x)| \leqq & C\left(r^{p-d} \int_{B}|D u-D v|^{p}+\left|\frac{u-v}{r}\right|^{p}\right)^{\frac{1}{p}\left(1-\frac{1}{2 p}\right)} \\
& \times\left(r^{-d} \int_{B}|u-v|^{p}\right)^{\frac{1}{p} \frac{1}{2 p}}
\end{aligned}
$$

almost everywhere $(B)$ for $p>d+\frac{1}{2}$. Then for skeletons of dimension $d>\lfloor p-1\rfloor$, where the embeddings no longer hold, a homogeneous degree 0 extension is used to inductively extend the lower-dimensional construction. The result of this construction on the triangulated domain yields an $f \in W^{1, p}\left(\mathcal{M} \times(0, \lambda), \mathbb{R}^{k}\right)$ such that $\int_{\mathcal{M} \times(0, \lambda)}|\nabla f|^{p} \leqq C \lambda A$ and

$$
\underset{\mathcal{M} \times(0, \lambda)}{\operatorname{ess} \sup } \operatorname{dist}(f, \mathcal{N}) \leqq c \lambda^{1-\frac{2 m+1}{2 p}} A^{\frac{1}{p}\left(1-\frac{1}{2 p}\right)}\left\|f_{1}-f_{2}\right\|_{p}^{\frac{1}{2 p}},
$$

where $A=\int_{\mathcal{M}}\left|D f_{1}\right|^{p}+\left|D f_{2}\right|^{p}+\left|\frac{f_{1}-f_{2}}{\lambda}\right|^{p}$. Now in the application of Luckhaus' lemma to regularity proofs, the need for the distance to be uniformly small is directly juxtapose with the control of $\operatorname{dist}(f, \mathcal{N})$ being derived from Sobolev embeddings (as outlined in the above). For the Dirichlet energy the conflict between these requirements is resolved by appealing to a monotonicity formula (cf. [78]). Indeed, for the class of convex integrands considered in [63] a monotonicity formula is also needed. In the $f(x, u, D u)$-situation considered here, higher integrability is required in order to carry out Ekeland's variational principle (note that the indirect approach of 
[34] no longer works with the $u$-dependence). In this regard the approach of [56], using connectedness assumptions on the target manifold, dispenses with the need for a monotonicity formula and allows for the application of Gehring's lemma.

The above Theorem 1.2 considers holonomic minimisers of quasiconvex integrals which also have $x$ - and $u$-dependences. Here we focus on the case $p<n$ (for if $p \geqq n$ the Sobolev embeddings imply the regularity argument would follow the single coordinate chart case). Our proof of Theorem 1.2 is direct via an application of Ekeland's variational principle; a certain tangential $\mathbb{A}$-harmonic approximate lemma; Caccioppoli inequalities using only minimality, the $p$-growth of (h1) and the strong quasiconvexity of (h2); the Sobolev lifting result of Theorem 4.1 below; and an extension-type result using the $\ell$-connectedness of the compact covering space $\widetilde{\mathcal{M}}$. The resulting estimates allow a sufficient decay of the excess functions $\mathcal{E}$ given by

$$
\mathcal{E}(r)=\mathcal{E}(x, r)=\left(f_{B_{r}(x)}\left|V\left(D u-(D u)_{x, r}\right)\right|^{2}\right)^{\frac{1}{2}}
$$

where $V(\cdot)$ is given by (9) below. ${ }^{3}$ Regularity follows via Campanato's integral characterisation of Hölder continuity [16, teor. I.2] with the singular set characterised by

$$
\begin{aligned}
\operatorname{Sing} u= & \left\{x \in \Omega: \limsup _{r \searrow 0}\left|(u)_{x, r}\right|=+\infty \text { or } \limsup _{r \searrow 0}\left|(D u)_{x, r}\right|=+\infty\right. \\
& \text { or } \underset{r \searrow 0}{\liminf } \mathcal{E}(x, r)>0\} .
\end{aligned}
$$

Remark 1.6. If we take a point $x \in \Omega \backslash \operatorname{Sing} u$ it follows that $\lim \sup \left|(u)_{x, r}\right|<$ $+\infty, \lim \sup \left|(D u)_{x, r}\right|<+\infty$ and $\lim \inf \mathcal{E}(x, r)=0$ by definition. Then there exists $\Lambda>0$ and $0<R<\operatorname{dist}(x, \partial \Omega)$ such that $\left|(u)_{x, r}\right|<\Lambda$ and $\left|(D u)_{x, r}\right|<\Lambda$ for all $0<r<R$. Similarly, for every $\varepsilon>0$ there exists $0<R<\operatorname{dist}(x, \partial \Omega)$ such that $\mathcal{E}(x, R)<\varepsilon$. Now if for every $\Lambda>0$ we can find $\varepsilon=\varepsilon(\Lambda)>0$ and $0<R=R(\Lambda)<\operatorname{dist}(x, \partial \Omega)$ both sufficiently small such that

$$
\mathcal{E}(x, R)<\varepsilon,
$$

then $x \in \Sigma_{\Lambda, \varepsilon, R}=\left\{y \in \Omega: \operatorname{dist}(y, \partial \Omega)>R, \mathcal{E}(y, R)<\varepsilon,\left|(D u)_{y, R}\right|<\right.$ $\left.\Lambda,\left|(u)_{y, R}\right|<\Lambda\right\}$. The set $\Sigma_{\Lambda, \varepsilon, R}$ is open (since for fixed $0<r \ll 1$ the maps $x \mapsto$ $\left|(u)_{x, r}\right|, x \mapsto\left|(D u)_{x, r}\right|$ and $x \mapsto \mathcal{E}(x, r)$ are continuous on $\{x \in \Omega: \operatorname{dist}(x, \partial \Omega)>$ $r\})$. Thus we need to show, for some $0<\beta<1$ and every $x \in \Sigma_{\Lambda, \varepsilon, R}$, that

$$
\mathcal{E}(x, r) \leqq K r^{\beta}, \quad 0<r<R,
$$

${ }^{3}$ We denote the average $(h)_{\Omega}=f_{\Omega} h=\frac{1}{|\Omega|} \int_{\Omega} h$ for any $h \in L^{1}\left(\Omega, \mathbb{R}^{N}\right)$, where $|\Omega|$ denotes the Lebesgue measure of any $\Omega \subset \mathbb{R}^{n}$, and use the abbreviation $(h)_{x, r}$, or simply $(h)_{r}$, for the mean value of $h$ on an open ball $B_{r}(x) \subset \mathbb{R}^{n}$ of radius $r$ centred at $x$. 
for some $K=K(\beta, \varepsilon, \Lambda, R)>0$, in order to conclude $D u \in C_{\text {loc }}^{0, \beta}\left(\Sigma_{\Lambda, \varepsilon, R}, \mathbb{R}^{N n}\right)$ by Campanato's integral characterisation of Hölder continuity. Consequently $D u$ is $C_{\mathrm{loc}}^{0, \beta}$-continuous on

$$
\begin{aligned}
& \operatorname{Reg} u=\bigcup_{\Lambda>0}\{x \in \Omega: \operatorname{dist}(x, \partial \Omega)>R(\Lambda), \\
&\left.\mathcal{E}(x, R)<\varepsilon(\Lambda),\left|(D u)_{x, R}\right|<\Lambda,\left|(u)_{x, R}\right|<\Lambda\right\},
\end{aligned}
$$

of which $\operatorname{Sing} u$ is the complement in $\Omega$, provided one has (7) and can establish the excess-decay (8).

In the upcoming analysis we make use of the following auxiliary function $V: \mathbb{R}^{k} \rightarrow \mathbb{R}^{k}$ defined by

$$
V(\xi)=\left(1+|\xi|^{2}\right)^{\frac{p-2}{4}} \xi
$$

for every $\xi \in \mathbb{R}^{k}$. Note that $\mathbb{R}^{+} \ni t \mapsto|V(t)|$ is convex and $|\xi|^{2},|\xi|^{p} \leqq|V(\xi)|^{2} \leqq$ $c(p)\left(|\xi|^{2}+|\xi|^{p}\right)$ when $p \geqq 2$. Also from the proof in [25, §4] we have, for any $v \in W^{1, p}\left(B_{R}, \mathbb{R}^{N}\right)$, the Sobolev-Poincaré-type inequality

$$
f_{B_{R}}\left|V\left(\frac{v-(v)_{R}}{R}\right)\right|^{2} \leqq c\left(f_{B_{R}}|V(D v)|^{2 *}\right)^{2 / 2_{*}}
$$

for some constant $c=c(n, N, p)>0$, where $B_{R} \subset \mathbb{R}^{n}, n \geqq 2$ and $2_{*}=\frac{2 n}{n+2}<2$. The following properties of (9) will also be called upon.

Lemma 1.7. ([17, p. 143], [6, p. 800]). Let $p \geqq 2$ and $V: \mathbb{R}^{k} \rightarrow \mathbb{R}^{k}$ be as in (9). Then for any $\xi, \eta \in \mathbb{R}^{k}$ and $t>0$ there holds:

$$
\begin{aligned}
\max \left\{|\xi|,|\xi|^{\frac{p}{2}}\right\} & \leqq|V(\xi)| \leqq 2^{\frac{p-2}{4}} \max \left\{|\xi|,|\xi|^{\frac{p}{2}}\right\} \\
|V(t \xi)| & \leqq \max \left\{t, t^{\frac{p}{2}}\right\}|V(\xi)| \\
|V(\xi+\eta)| & \leqq c(p)(|V(\xi)|+|V(\eta)|) \\
c(p)|\xi-\eta| & \leqq \frac{|V(\xi)-V(\eta)|}{\left(1+|\xi|^{2}+|\eta|^{2}\right)^{\frac{p-2}{4}}} \leqq C(k, p)|\xi-\eta| \\
|V(\xi)-V(\eta)| & \leqq c(k, p, \Lambda)|V(\xi-\eta)|, \quad \text { whenever }|\eta| \leqq \Lambda \\
|V(\xi-\eta)| & \leqq c(p)|V(\xi)-V(\eta)| .
\end{aligned}
$$

\subsection{Applications to Problems in Condensed Matter Physics}

The manifold constrained regularity problem has several applications to problems in condensed matter physics. For such applications the (unconstrained) mapping is a tensor order parameter $T=\left(T_{\alpha \beta}\right): \Omega \subset \mathbb{R}^{n} \rightarrow \mathbb{R}^{N}$ with an integral functional of the form

$$
\int_{\Omega}\left(f_{\text {grad }}(T, \nabla T)+f_{B}(T)\right) \mathrm{d} x .
$$


The integrand is taken to be invariant under a given symmetry group $G$ and is typically expanded as power series with respect to this symmetry group. Critical points of the bulk energy $f_{B}$ form an orbit of solutions corresponding to a subgroup $H \subset G$ of residual symmetries. The resulting manifold of internal states $\mathcal{M}=$ $G / H$ describes the 'broken symmetries' of the system; such $T$ taking values in this group orbit (for which the bulk energy $f_{B}$ remains constant) form the corresponding constrained mapping case.

Example 1.8. (Nematic liquid crystals) In the Landau-de Gennes theory for nematic liquid crystals the state of alignment is characterised by a tensor order parameter $Q$ as a traceless symmetric $3 \times 3$ real matrix, that is $Q=\left(Q_{i j}\right): \Omega \subset \mathbb{R}^{3} \rightarrow$ $\operatorname{Sym}_{0}^{2}\left(\mathbb{R}^{3}\right) \equiv \mathbb{R}^{5}$. In a general nematic phase the order parameter has five degrees of freedom (two specifying the degree order with the other three for the principal directions). The symmetry group is $G=\mathrm{SO}(3)$ with the group action $\star$ defined by conjugation

$$
R \star Q_{i j}=R_{i k} R_{j \ell} Q_{k \ell}
$$

for any $R \in \mathrm{SO}$ (3). The Landau-de Gennes free-energy functional (cf. [20]), taking into consideration spatial variations of the local order parameter, is of the form

$$
\mathcal{F}(Q ; \Omega)=\int_{\Omega}\left(f_{\text {grad }}(Q, \nabla Q)+f_{B}(Q)\right) \mathrm{d} x .
$$

The bulk free energy $f_{B}$ is invariant under the $\mathrm{SO}(3)$-action by conjugation on the five-dimensional space of $Q$-tensors; its expansion to fourth order takes the form

$$
f_{B}(Q)=-\frac{a^{2}}{2} \operatorname{tr} Q^{2}-\frac{b^{2}}{3} \operatorname{tr} Q^{3}+\frac{c^{2}}{4}\left(\operatorname{tr} Q^{2}\right)^{2},
$$

where $a, b$ and $c$ are material and temperature dependent constants (cf. [66]). The expansion of the free energy $f_{\text {grad }}$ in $\mathrm{SO}(3)$-invariant powers of $Q_{i j}$, and its derivatives up to the order $Q Q \nabla Q \nabla Q$, contains twenty-two terms and four surface relations (cf. $[11,65,81]$ ). For instance the lowest order terms of the form $\nabla Q \nabla Q$ are

$$
Q_{i j, k} Q_{i j, k} \quad Q_{i j, j} Q_{i k, k} \quad Q_{i j, k} Q_{i k, j}
$$

with the corresponding surface relation

$$
\partial_{i}\left(Q_{i j} Q_{j k, k}-Q_{j k} Q_{i j, k}\right)=Q_{i j, j} Q_{i k, k}-Q_{i j, k} Q_{i k, j} \cdot{ }^{4}
$$

Note that there are only five possible contractions of a symmetric 2-tensor in the form $\nabla Q \nabla Q$; the other remaining two terms $Q_{k k, j} Q_{i i, j}$ and $Q_{i i, j} Q_{j k, k}$ are excluded since the matrix is traceless. Critical points of the bulk energy form an orbit of solutions in the five-dimensional space of $Q$-tensors. Such $Q$ taking values in a group orbit have constant eigenvalues and constant bulk energy. We refer to this situation as the constrained theory of nematic liquid crystals. In the constrained

${ }^{4}$ Here we denote $Q_{i j, k}=\partial_{k} Q_{i j}$. As usual, summation over repeated indices is assumed. 
uniaxial case (that is when the $Q$-tensor has two equal non-zero eigenvalues) the manifold of internal states is equal to $\mathrm{SO}(3) / D_{\infty}=\mathbb{R} P^{2}$, using infinite dihedral group $D_{\infty}$, which can be identified with the homogeneous space $\mathrm{SO}(3) / O(2)$ (cf. [43, ex. 2.45]); whereas the corresponding space of constrained biaxial $Q$ tensors (that is $Q$-tensors having three distinct eigenvalues) is equal to $\mathrm{SO}(3) / D_{4}=$ $S^{3} / \mathbb{H}$, where $D_{4}$ denotes the dihedral group consisting of the identity together with $\pi$-rotations about three mutually perpendicular axes and $\mathbb{H}$ denotes the quaternion group (cf. [73]).

Example 1.9. (Superfluidity of liquid helium-3) There are two stable isotopes of the chemical element helium, namely ${ }^{3} \mathrm{He}$ and ${ }^{4} \mathrm{He}$. At 1 atm helium-3 boils at $3.19 \mathrm{~K}$ compared with helium- 4 at $4.23 \mathrm{~K}$. Below these temperatures one finds helium liquids, unlike all other known liquids, do not solidify unless a pressure of around 30 bar is applied-under less pressure the small atomic mass and the weakness of the attractive interactions prevents the formation of a crystalline solid with a rigid lattice structure. This is the first indication of the remarkable macroscopic quantum effects in these systems. Quantum effects are also responsible for the strikingly different behaviours of ${ }^{3} \mathrm{He}$ (obeying Fermi-Dirac statistics) and ${ }^{4} \mathrm{He}$ (obeying Bose-Einstein statistics) at lower temperatures: the superfluid transition temperature of helium- 4 is $2.17 \mathrm{~K}$ at 1 atm, whereas for helium-3 it is below $3 \mathrm{mK}$ and in fact forms several superfluid phases. ${ }^{5}$ In the case of superfluid helium-3, the order parameter $A=\left(A_{\mu j}\right)$ is a $3 \times 3$ complex valued matrix, that is $A: \Omega \subset$ $\mathbb{R}^{3} \rightarrow \mathbb{C}^{3} \otimes \mathbb{C}^{3} \equiv \mathbb{R}^{18}$, with spin index $\mu$ and orbital index $j$. The associated symmetry group $G=\mathrm{SO}(3) \times \mathrm{SO}(3) \times U(1)$ with the group action $\star$ defined by

$$
\left(R^{(1)}, R^{(2)}, \phi\right) \star A_{\mu j}=e^{i \phi} R_{\mu \nu}^{(1)} R_{j k}^{(2)} A_{\nu k}
$$

for a phase $\phi$ with rotation matrices $R^{(1)}$ and $R^{(2)}$ in the respective spin space $S$ and orbital space $\boldsymbol{L}$. In regions near the critical temperate $T_{c}$ (that is the GinzburgLandau regime) the bulk free energy $f_{B}$ can be obtained from $A_{\mu j}$ by contracting all indices in second and fourth order terms that are $\mathrm{SO}(3) \times \mathrm{SO}(3) \times U(1)$-invariant. The resulting expansion yields

$$
\begin{aligned}
f_{B}= & -\alpha A_{\mu j}{ }^{*} A_{\mu j}+\beta_{1} A_{\mu j}{ }^{*} A_{\mu j}{ }^{*} A_{\nu \ell} A_{\nu \ell}+\beta_{2} A_{\mu j}{ }^{*} A_{\mu j} A_{\nu \ell}{ }^{*} A_{\nu \ell} \\
& +\beta_{3} A_{\mu j}{ }^{*} A_{\nu j}{ }^{*} A_{\mu \ell} A_{\nu \ell}+\beta_{4} A_{\mu j}{ }^{*} A_{\nu j} A_{\nu \ell}{ }^{*} A_{\mu \ell}+\beta_{5} A_{\mu j}{ }^{*} A_{\nu j} A_{\nu \ell} A_{\mu \ell},
\end{aligned}
$$

where the coefficients $\alpha$ and $\beta_{i}$ depend on external parameters like temperature and pressure (cf. [74]). When allowing for spatial variations in the order parameter, the gradient energy of an anisotropic superfluid in the Ginzburg-Landau regime has leading $\mathrm{SO}(3) \times \mathrm{SO}(3) \times U(1)$-invariant terms quadratic in the gradient of the form

$$
f_{\mathrm{grad}}=\gamma_{1} \partial_{j} A_{\mu \ell} \partial_{j} A_{\mu \ell}{ }^{*}+\gamma_{2} \partial_{j} A_{\mu \ell} \partial_{\ell} A_{\mu j}{ }^{*}+\gamma_{3} \partial_{j} A_{\mu j} \partial_{\ell} A_{\mu \ell}{ }^{*},
$$

${ }^{5}$ When the superfluid phases of ${ }^{3} \mathrm{He}$ were finally discovered $[75,76]$ at temperatures of 2.6 and $1.8 \mathrm{mK}$ respectively - in an experiment actually designed to observe a magnetic phase transition in solid ${ }^{3} \mathrm{He}$ - the results came as a great surprise. 
Table 1. The manifold of broken symmetries $\mathcal{M}$ and universal cover (UC) of some examples of ordered media

\begin{tabular}{llll}
\hline $\mathcal{M}$ & $\pi_{1}$ & $\mathrm{UC}$ & \\
\hline$S^{1}$ & $\mathbb{Z}$ & $\mathbb{R}$ & Planar spins/superfluid ${ }^{4} \mathrm{He}$ \\
$S^{1} \times S^{1}$ & $\mathbb{Z}+\mathbb{Z}$ & $\mathbb{R}^{2}$ & Dipole-locked ${ }^{3}$ He-A in weak magnetic field \\
$S^{2}$ & 0 & $S^{2}$ & Ordinary spins/ferromagnet \\
$\mathbb{R} P^{2}$ & $\mathbb{Z}_{2}$ & $S^{2}$ & Uniaxial nematics \\
$S^{3} / \mathbb{H}$ & $\mathbb{H}$ & $S^{3}$ & Biaxial nematics \\
$\mathbb{R} P^{3}=\mathrm{SO}(3)$ & $\mathbb{Z}_{2}$ & $S^{3}$ & Dipole-locked ${ }^{3} \mathrm{He}-\mathrm{A}$ \\
$S^{1} \times \mathrm{SO}(3)$ & $\mathbb{Z}^{3} \mathbb{Z}_{2}$ & $\mathbb{R} \times S^{3}$ & ${ }^{3}$ He-B \\
$\left(S^{1} \times \mathrm{SO}(3)\right) / \mathbb{Z}_{2}$ & $\mathbb{Z}+\mathbb{Z}_{4}$ & $\mathbb{R} \times S^{3}$ & Dipole-free ${ }^{3} \mathrm{He}-\mathrm{A}$ in strong magnetic field \\
$\left(S^{2} \times \mathrm{SO}(3)\right) / \mathbb{Z}_{2}$ & $\mathbb{Z}_{4}$ & $S^{2} \times S^{3}$ & Dipole-free ${ }^{3} \mathrm{He}-\mathrm{A}$ \\
\hline
\end{tabular}

Further information on broken symmetries with regards to gradient, dipole and magnetic interactions can be found in $[67,80,82]$

where $\gamma_{i}$ are given constants (cf. [1,21]). Note that the terms are dependent in the sense that the second term can be written in terms of the third plus a divergence terms which vanishes under appropriate boundary conditions. The unusually large number of internal degrees of freedom allow for a rich array of superfluid phases of liquid ${ }^{3} \mathrm{He}$. Fortunately it is possible to formulate a systematic approach to the investigation of all possible symmetry breakings; the classification of all continuous and discrete subgroups of $\mathrm{SO}(3) \times \mathrm{SO}(3) \times U(1)$ can be found in [15]. It turns out that the continuous subgroup with the highest remaining symmetry corresponds to the so-called $B$-phase of [76] that is stable down to the lowest temperatures attained so far. In this phase the order parameter is invariant under joint rotations of $\boldsymbol{L}$ and $S$ represented by the subgroup $\mathrm{SO}(3)_{L+S}$. The corresponding broken symmetries are $\mathcal{M}_{B}=\frac{\mathrm{SO}(3)_{L} \times \mathrm{SO}(3)_{S}}{\mathrm{SO}(3)_{L+S}} \times U(1)_{\phi}=\mathrm{SO}(3)_{L, S} \times S_{\phi}^{1}$. On the other hand, if one is confined to a finite temperature range and is above a critical pressure of about 21 bar, one enters a different phases: the so-called $A$-phase of [76]. In this dipolefree $A$-phase the subgroup of residual symmetries is $U(1)_{L_{z}-\phi} \times U(1)_{S_{z}} \times \mathbb{Z}_{2}$. The corresponding space of broken symmetries is the five dimensional manifold $\mathcal{M}_{A}=\left(\frac{\mathrm{SO}(3)_{S}}{U(1)_{L}} \times \frac{\mathrm{SO}(3)_{L} \times U(1)_{\phi}}{U(1)_{L_{z}-\phi}}\right) / \mathbb{Z}_{2}=\left(S_{S}^{2} \times \mathrm{SO}(3)_{L, \phi}\right) / \mathbb{Z}_{2}$. Other manifolds of broken symmetries can be considered if an external magnetic field is also considered (cf. Table 1). For a review of the theory of anisotropic superfluid phases of helium-3 see [62].

\section{Submanifolds, Projections and the Distance Function}

\subsection{Tubular Neigbourhoods}

For an embedded submanifold $\mathcal{M}$ in $\mathbb{R}^{N}$ without boundary, and its normal bundle $\pi:(T \mathcal{M})^{\perp} \rightarrow \mathcal{M}$, define the 'endpoint map' $E:(T \mathcal{M})^{\perp} \rightarrow \mathbb{R}^{N}$ by sending $(x, v) \mapsto x+v$. A tubular neighbourhood of $\mathcal{M}$ is an open neighbourhood $\mathcal{O}$ of $\mathcal{M}$ in $\mathbb{R}^{N}$ that is the diffeomorphic image under the map $E$ of an open neighbourhood $\mathscr{O} \subset(T \mathcal{M})^{\perp}$ of the zero section $0: \mathcal{M} \rightarrow(T \mathcal{M})^{\perp}$. As the differential $E_{*}$ 
is surjective along this zero section, $E$ is a local diffeomorphism by the inverse function theorem. One then has the following existence result:

Theorem 2.1. Let $\mathcal{M}$ be a compact submanifold of $\mathbb{R}^{N}$ without boundary, then there exists a tubular neighbourhood $\mathcal{O}$ of $\mathcal{M}$ in $\mathbb{R}^{N}$.

By identifying $\mathcal{M}$ with its image in $\mathbb{R}^{N}$ we say that a neighbourhood $\mathcal{O}$ of $\mathcal{M}$ has the unique nearest point property if for every $x \in \mathcal{O}$ there is a unique point $\Pi(x) \in \mathcal{M}$ such that $\operatorname{dist}(x, \mathcal{M})=|x-\Pi(x)|$. The map

$$
\Pi: \mathcal{O} \rightarrow \mathcal{M}
$$

is called the retraction onto $\mathcal{M}$.

Lemma 2.2. Let $\mathcal{M}$ be a compact $C^{r}$-submanifold of $\mathbb{R}^{N}$ without boundary for $r \geqq 2$. Then there exists a neighbourhood $\mathcal{O}$ of $\mathcal{M}$ with the unique nearest point property and the retraction $\Pi: \mathcal{O} \rightarrow \mathcal{M}$ is $C^{r-1}$.

Proof. The existence of $\mathcal{O}$ follows from Theorem 2.1. As the normal bundle $\pi:(T \mathcal{M})^{\perp} \rightarrow \mathcal{M}$ is $C^{r-1}$ it follows that $E$ is a $C^{r-1}$-diffeomorphism. Hence the map $\Pi=\pi \circ E^{-1}$ is $C^{r-1}$.

A focal point $y$ at $x \in \mathcal{M}$ with multiplicity $v$ occurs if $y=E(x, v)$, where $v$ is normal to $\mathcal{M}$ at $x$, and the differential $\left.E_{*}\right|_{(x, v)}$ is a critical point with nullity $v>0$; they are points where nearby normals intersect. By [68, Lem. 6.3] these focal points are precisely of the form $x+\kappa_{i}^{-1} v$, where $\kappa_{i}$ are the principal curvatures of $\mathcal{M}$ at $x$ in the unit normal direction $v$.

Remark 2.3. As $W_{\text {loc }}^{r-1, \infty}\left(\mathcal{O}, \mathbb{R}^{N}\right)=C^{r-2,1}\left(\mathcal{O}, \mathbb{R}^{N}\right)$ are the locally Lipschitz functions, it follows for each compact set $K \subset \mathcal{O}$ there exists $C=C(K)>0$ such that

$$
\left|\nabla^{(j)} \Pi(x)-\nabla^{(j)} \Pi(y)\right| \leqq C|x-y|
$$

for all $x, y \in K$ where $j=0, \ldots, r-2$ (cf. [35, §4.2.3]). For instance, one could take $K$ to be the closure of the tubular neighbourhood $\{x+v: x \in \mathcal{M}, v \in$ $\left.\left(T_{x} \mathcal{M}\right)^{\perp},|v|<\varepsilon\right\}$, for $\varepsilon>0$ sufficiently small, so as to remain 'strictly away from focal points'. Furthermore, each $\nabla^{(j)} \Pi$ are uniformly bounded and uniformly continuous on compact sets for $j=0, \ldots, r-1$.

Note that the boundary of a manifold cannot have a tubular neighbourhood strictly in the above sense. However, there is a retraction of an open neighbourhood onto $\partial \mathcal{M}$. This is obviously possible locally, and two local retractions into coordinate domains can be glued together with a bump function. 


\subsection{Sets of Positive Reach and the Regularity of the Squared Distance Function}

For a subset $A \subset \mathbb{R}^{N}$ let the distance function $\delta: \mathbb{R}^{N} \rightarrow \mathbb{R}$ be defined by

$$
\delta(x)=\operatorname{dist}(x, A)=\inf \{|x-y|: y \in A\} .
$$

Furthermore, let $\operatorname{Unp}(A)$ be the set of all points $x \in \mathbb{R}^{N}$ for which there exists a unique point of $A$ nearest to $x$ and, like in the previous section, let the map $\Pi: \operatorname{Unp}(A) \rightarrow A$ associate to $x \in \operatorname{Unp}(A)$ the unique $a \in A$ such that $\delta(x)=$ $|x-a|$.

If $a \in A$ then $\operatorname{reach}(A, a)$ is the supremum of the set of all numbers $r$ for which $\{x:|x-a|<r\} \subset \operatorname{Unp}(A)$. We then define $\operatorname{reach}(A)=\inf \{\operatorname{reach}(A, a): a \in A\}$. If $\operatorname{reach}(A)>0$ then it is said that $A$ has positive reach. A well known characterisation of convexity (going back to independent results by BUNT, KRITIKOS and Motzkin, cf. [19]) states that a subset $A \subset \mathbb{R}^{N}$ has reach $(A)=+\infty$ if and only if $A$ is closed and convex.

By introducing the squared distance function $\eta(x)=\frac{1}{2} \delta(x)^{2}$ we have:

Theorem 2.4. $[36,4.8]$ For every nonempty closed subset $A$ of $\mathbb{R}^{N}$ the following statements hold with $U=\operatorname{Unp}(A)$ :

1. $|\delta(x)-\delta(y)| \leqq|x-y|$ for all $x, y \in \mathbb{R}^{N}$.

2. If $x \in \mathbb{R}^{N} \backslash A$ and $\delta$ is differentiable at $x$, then $x \in U$ and $\nabla \delta(x)=\frac{x-\Pi(x)}{\delta(x)}$.

3. П is continuous.

4. $\delta$ is continuously differentiable on $\operatorname{Int}(U \backslash A)$ and $\eta$ is continuously differentiable on $\operatorname{Int}(U)$ with $\nabla \eta(x)=x-\Pi(x)$ for $x \in \operatorname{Int}(U)$.

5. If for $0<r<s<\infty$ and $x, y \in U$ we have $\delta(x) \leqq r, \delta(y) \leqq r$ and $\operatorname{reach}(A, \Pi(x)) \geqq s, \operatorname{reach}(A, \Pi(y)) \geqq s$, then $|\Pi(x)-\Pi(y)| \leqq \frac{s}{s-r}|x-y|$.

6. If $0<q<r<\operatorname{reach}(A)$ then $\nabla \delta$ is Lipschitzian on $\{x: q \leqq \delta(x) \leqq r\}$ and $\nabla \eta$ is Lipschitzian on $\{x: \delta(x) \leqq r\}$.

For a compact $C^{r}$-submanifold $\mathcal{M} \subset \mathbb{R}^{N}$ without a boundary, the distance function $\delta$ is $C^{r-1}$ near $\mathcal{M}$ (since it is continuous and can be written in terms of directions normal to $\mathcal{M}$ ); in fact the squared distance function $\eta \in C^{r}(U)$ for $r \geqq 2$ (cf. [38], [47, p. 354]). Furthermore, by approximating $\mathcal{M}$ locally at a point $x$ as the graph of a quadratic polynomial given by the second fundamental form, one can show for any $z \in \mathbb{R}^{N}$ with $0<|z| \ll 1$ sufficiently small, using the elementary geometry of the normal line at $\Pi(x+z)$ through $x+z$ and the normal line at $x$ along the projection of $z$ onto $\left(T_{x} \mathcal{M}\right)^{\perp}$, that

$$
\eta(x+z)=\frac{1}{2}|Q z|^{2}+O\left(|z|^{3}\right),
$$

where the matrix $Q$ represents the orthogonal projection onto the normal space of $\mathcal{M}$ at the point $x$. On the other hand, the Taylor series of $\eta$ is $\eta(x+z)=$ $\frac{1}{2}\left(\nabla^{2} \eta\right)(x)(z, z)+O\left(|z|^{3}\right)$, since $\eta(x)=0$ and $\nabla \eta(x)=0$ due to the projection $\Pi(x)=x$. Therefore $\nabla^{2} \eta$ represents the orthogonal projection onto the normal space of $\mathcal{M}$ and $P=I-\nabla^{2} \eta$ is the projection operator onto the tangent space. Moreover, by taking a further derivative of (4) in Theorem 2.4 we conclude: 
Corollary 2.5. The symmetric 2-tensor $P(x)=\nabla \Pi(x)$ projects vectors at a point $x \in \mathcal{M}$ onto the corresponding tangential subspace $T_{x} \mathcal{M}$.

Example 2.6. For the $n$-sphere $S^{n} \subset \mathbb{R}^{n+1}$ the retraction $\Pi: x \mapsto \frac{x}{|x|}$ for $x \neq 0$. Thus one finds that $P(u)=\nabla \Pi(u)=I-u \otimes u$ at points $u \in S^{n}$, where $I$ is the identity matrix.

\section{Constrained Stationary Variations}

Consider the functional

$$
\mathcal{F}(v ; \Omega)=\int_{\Omega} F(D v) \mathrm{d} x
$$

where $v: \Omega \subset \mathbb{R}^{n} \rightarrow \mathbb{R}^{N}$. For a compact $C^{3}$-Riemannian submanifold $\mathcal{M} \subset$ $\mathbb{R}^{N}$ without boundary, we derive the Euler-Lagrange equations for holonomically constrained minimisers, using the retraction (12), as follows:

Lemma 3.1. [34, p. 78]. Suppose the integrand $F: \mathbb{R}^{N n} \rightarrow \mathbb{R}$ is a $C^{1}$-function that for $p>1$ satisfies

$$
|D F(\xi)| \leqq C\left(1+|\xi|^{p-1}\right)
$$

for some constant $C>0$ and all $\xi \in \mathbb{R}^{N n}$. Then for any holonomic $\mathcal{F}$-minimiser $u \in W_{\text {loc }}^{1, p}(\Omega, \mathcal{M})$ we have

$$
\int_{\Omega} D F(D u) D(\nabla \Pi(u) \phi) \mathrm{d} x=0
$$

for all $\phi \in W_{0}^{1, p}\left(\Omega, \mathbb{R}^{N}\right) \cap L^{\infty}\left(\Omega, \mathbb{R}^{N}\right)$.

Example 3.2. When considering the Dirichlet energy $\int_{\Omega}|D u|^{2} \mathrm{~d} x$ the above lemma implies holonomically constrained minimisers satisfy

$$
\int_{\Omega}\left\langle D u, \nabla \Pi(u) D \phi+\nabla^{2} \Pi(u) D u \cdot \phi\right\rangle \mathrm{d} x=0 .
$$

One then concludes

$$
\Delta u+A(u)(D u, D u)=0
$$

weakly, where $A$ is the second fundamental form of the embedding of $\mathcal{M}$ in $\mathbb{R}^{N}$ (cf. [50, Lem. 1.2.4]). Such solutions to (13) are said to be weakly harmonic maps. 


\section{A Construction of Suitable Comparison Maps}

In seeking to prove the regularity of vector-valued maps which minimise an integral functional subject to a family of smooth nonlinear (manifold) constraints, a major technical difficulty resides in finding comparison maps which satisfy the manifold constraints (particularly since one cannot localise the problem in the image for $n>2$ ). In the case of harmonic maps into compact manifolds, one can construct comparison maps via a lemma of Luckhaus originally proved in [63, Lem. 1] and revised in [64, Lem. 3] (which develops an earlier extension lemma of [79, Lem. 4.3]). Using this and a monotonicity formula for harmonic maps it is possible to prove Caccioppoli inequalities near points with small scale energy (cf. [78, §2.8]). However, without such a monotonicity formula available in general one needs to construct comparison maps absent of any need for initial 'smallness assumptions'. Our approach here is to combine the lifting of Sobolev maps to the universal covering together with an extension result using the connectedness of the covering space.

\subsection{The Lifting of Sobolev Mappings}

For a given connected compact Riemannian manifold $\mathcal{M}$ without boundary and an open simply connected subset $\Omega \subset \mathbb{R}^{n}$ we ask if any mapping $u \in W^{s, p}(\Omega, \mathcal{M})$ factors through the universal covering $\pi: \widetilde{\mathcal{M}} \rightarrow \mathcal{M}$ as regular as $u$ permits, that is if

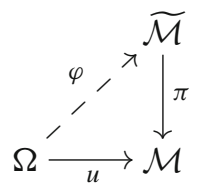

can be completed to a commutative diagram with $\varphi \in W^{s, p}(\Omega, \widetilde{\mathcal{M}})$. Of course if $\mathcal{M}$ is simply connected then $\widetilde{\mathcal{M}}=\mathcal{M}$ and $\pi$ is the identity map. Hence we are concerned with the non-trivial case $\pi_{1}(\mathcal{M}) \neq 0$.

For such a problem we have the following affirmative result when $2 \leqq p \leqq \infty$ and $s=1$.

Theorem 4.1. [9] Let $\Omega \subset \mathbb{R}^{n}$ be an open simply connected subset, $\mathcal{M}$ be a smooth connected compact Riemannian manifold without boundary and $\pi: \widetilde{\mathcal{M}} \rightarrow \mathcal{M}$ be its universal covering. For every $2 \leqq p \leqq \infty$ and any $u \in W^{1, p}(\Omega, \mathcal{M})$ there exists $\varphi \in W^{1, p}(\Omega, \widetilde{\mathcal{M}})$ such that $u=\pi \circ \varphi$. Moreover, $\varphi$ is unique up to the action of an element of $\pi_{1}(\mathcal{M})$ and satisfies $|D u|=|D \varphi|$ for almost everywhere $x \in \Omega$.

\subsection{A Locally Lipschitz Retraction}

We recall some basic notions pertaining to the triangulation of a manifold. Firstly, we say a cell $\Delta$ is a bounded subset of $\mathbb{R}^{m}$ consisting of all points $x$ satisfying a system of finite linear inequalities $\ell_{i}(x)=\sum a_{i j} x_{j} \geqq b_{i}$; it is a compact convex 
subset of $\mathbb{R}^{m}$. The dimension of $\Delta$ is the dimension of the smallest dimensional plane containing $\Delta$. If $\Delta$ is an $m$-cell, $\partial \Delta$ (the boundary of $\Delta$ ) is the union of a finite number of $(m-1)$-cells, each the intersection of an $(m-1)$-plane with $\Delta$. Each of the $(m-1)$-cells $\Sigma_{i}$ into which $\partial \Delta$ is decomposed is called a face of $\Delta$; each of the $(m-2)$-faces of $\Sigma_{i}$ is called an $(m-2)$-face of $\Delta$. As $\Delta$ is given by a system of linear inequalities, replacing an inequality by equality determines a face of $\Delta$ and conversely.

Definition 4.2. A cell complex $K$ is a collection of cells such that each face of a cell in $K$ is also in $K$, the intersection of two cells in $K$ is either empty or a common face, and each point of the polyhedron (as the union of all cells of $K$ ) has a neighbourhood intersecting finitely many cells of $K$. The dimension of $K$ is the maximal dimension of the cells in $K$.

A subcomplex $K^{\prime}$ is a subset of $K$ which itself is a complex; to verify a subcomplex is indeed a complex it is clear that one only needs to check that faces of each cell in $K^{\prime}$ are in $K$. In particular, the $k$-skeleton $K^{k}$ is the subcomplex of $K$ consisting of all cells of dimension at most $k$. In the case when the cell is a cube in $\mathbb{R}^{m}$ centred at the origin with faces parallel to the coordinate axes, the dual skeleton of dimension $j$ is the set of points in the cube which have at least $m-j$ components equal to zero. By using an isometry, the dual skeleton of a cube in $\mathbb{R}^{m}$ of dimension $m$ can be defined in a general position. Thus the $j$-dimensional dual skeleton $L^{j}$ of a complex $K$ of cubes is simply the union of the dual skeletons of dimension $j$ of each cube in the complex (cf. [12, §2.1]).

A Lipschitz triangulation $f: K \rightarrow \mathcal{M}$ of a given manifold $\mathcal{M}$ can be such that the complex $K$ (given by Definition 4.2) consists of cubes each of which is isometric to the standard cube $[-1,1]^{m} \subset \mathbb{R}^{m}$ (that is $K$ is a 'cubication' of $\mathcal{M}$ ). ${ }^{6} \mathrm{~A}$ result by WHITEHEAD [85, Theor. 7] states that any compact $C^{1}$-manifold without boundary has a triangulation in the $C^{1}$-class. This was extended by MunKREs [72, Chap. 2] to include $C^{r}$-manifolds with boundary for $1 \leqq r \leqq \infty$. In particular:

Theorem 4.3. Every compact $C^{r}$-manifold without boundary has a $C^{r}$-triangulation. If $\mathcal{M}$ is a manifold having boundary, any $C^{r}$-triangulation of the boundary may be extended to a $C^{r}$-triangulation of $\mathcal{M}$.

Lemma 4.4. [83, p. 130] Let $K$ be a cell complex of dimension $n$ consisting of cubes each of which is isometric to the standard cube. Let $L^{n-\ell-1}$ be the $(n-\ell-1)$ dimensional dual skeleton of $K$ for some integer $0 \leqq \ell \leqq n-1$. Then there exists a locally Lipschitz continuous function

$$
\psi: K^{n} \backslash L^{n-\ell-1} \rightarrow K^{\ell}
$$

${ }^{6}$ Such a cubication is always possible: for if we consider the $k$-simplex $\Delta=$ $\left\{\left(x_{1}, \ldots, x_{k+1}\right): \sum x_{i}=1, x_{i} \geqq 0\right\}$ in $\mathbb{R}^{k+1}$, the map $x \mapsto \frac{x}{\max \left|x_{i}\right|}$ projects $\Delta$ out onto faces of $[0,1]^{k+1}$ containing $(1, \ldots, 1)$ and so divides it into $(k+1)$-cubes. Hence if $\mathcal{M}$ has already been triangulated, one can connect the centroid of each triangle to the midpoints of its sides forming three 'squares'. Then by connecting the centroid of each 3-simplex to the centroids of each of its four faces we divide the 3-simplex into four 3-dimensional cubes, etc. (cf. [83, p. 129]). 
from the complement of the dual skeleton $L^{n-\ell-1}$ onto the skeleton $K^{\ell}$ such that

$$
|D \psi(x)| \leqq \frac{C}{\operatorname{dist}\left(x, L^{n-\ell-1}\right)}
$$

for some constant $C>0$ depending on $n, \ell$ and $K$.

Proof. For a cube $[-1,1]^{n}$ define the function $|\cdot|_{\ell}:[-1,1]^{n} \rightarrow \mathbb{R}$ by

$$
|x|_{\ell}=\min _{\substack{S \subset\{1, \ldots, n\} \\|S|=\ell+1}} \max _{i \in S}\left|x_{i}\right| .
$$

If $L^{n-\ell-1}$ denotes the $(n-\ell-1)$-dual skeleton of $[-1,1]^{n}$ we have $x \in L^{n-\ell-1}$ if and only if $|x|_{\ell}=0$. Moreover, the mapping $[-1,1]^{n} \ni x \mapsto|x|_{\ell}$ is Lipschitz continuous with Lipschitz constant 1 . Using this we can define the mapping

$$
\phi_{\ell}:[-1,1]^{n} \backslash L^{n-\ell-1} \rightarrow K^{\ell}
$$

by $\phi_{\ell}(x)=\left(y_{1}, \ldots, y_{n}\right)$, where $y_{i}=\operatorname{sgn} x_{i}$ if $\left|x_{i}\right| \geqq|x|_{\ell}$ and $y_{i}=x_{i} /|x|_{\ell}$ if $\left|x_{i}\right|<|x|_{\ell}$. Observe also that $\left|D \phi_{\ell}(x)\right| \leqq C /|x|_{\ell}$. Given this, the same construction can be performed for every cube in $K$. If two cubes have a non-empty intersection the corresponding maps $\phi_{\ell, 1}$ and $\phi_{\ell, 2}$ coincide on the common face. Hence we can glue together the locally Lipschitz continuous maps obtained for each of the cubes in the complex $K$ so as to obtain a global map $\psi$ with the desired properties.

Lemma 4.5. Let $\mathcal{M} \subset \mathbb{R}^{k}, k \geqq 3$, be a compact $\ell$-connected submanifold, for some integer $1 \leqq \ell \leqq k-2$, contained in a $k$-dimensional cube $Q$. Then there exists a closed $(k-\ell-2)$-dimensional Lipschitz polyhedron $X \subset Q \backslash \mathcal{M}$ and $a$ locally Lipschitz retraction $\Theta: Q \backslash X \rightarrow \mathcal{M}$ such that for any $x \in Q \backslash X$,

$$
|D \Theta(x)| \leqq \frac{C}{\operatorname{dist}(x, X)}
$$

for some constant $C>0$ depending on $k, \ell$ and $\mathcal{M}$.

This result was first proved in [56, Lem. 6.1] using several results from homotopy theory (see also [13, Lem. 2.2]). Our proof below is based on the elementary result, for a Riemannian manifold $Y$, that

Proposition 4.6. $\pi_{n}\left(Y, y_{0}\right)=0$ for all $y_{0} \in Y$ if and only if every Lipschitz map $S^{n} \rightarrow Y$ admits a Lipschitz extension $B^{n+1} \rightarrow Y$.

This extension result follows from the fact that continuous mappings and homotopies can be approximated by smooth ones (cf. [61, Theor. 6.26]), however if the smooth manifold $Y$ is replaced by a metric space the result no longer holds (cf. [26, $\S 4])$. 


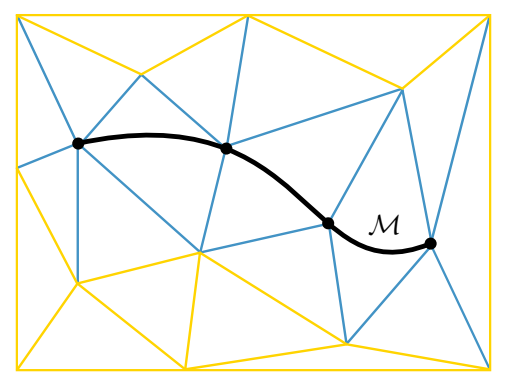

Fig. 1. An extension of a triangulation of a manifold $\mathcal{M} \subset Q$ to a triangulation of the cube $Q$. Simplexes intersecting with $\mathcal{M}$ are shown in blue whereas ones that are not are in yellow (color figure online)

Proof of Lemma 4.5. Let $f: T \rightarrow \mathcal{M}$ be a Lipschitz triangulation of the $m$ dimensional manifold $\mathcal{M}$. As $\mathcal{M}$ is contained in a $k$-dimensional cube $Q$, we can extend the triangulation $T$ of $\mathcal{M}$ to a Lipschitz triangulation $F: K \rightarrow Q$ of the cube $Q$.

Now consider all edges $\Delta^{1}$ of the triangulation $K$ that meet the complex $T^{m}$ of $\mathcal{M}$ at one or both vertices such that the edge is not contained in $T^{m}$ (cf. Fig. 1). If we send the vertex not in $T^{m}$ of any such $\Delta^{1}$ to any point in $\mathcal{M}$, we have a mapping $\phi^{0}: S^{0} \rightarrow \mathcal{M}$. Since $\pi_{0}(\mathcal{M})=0$ there exists $\Phi^{0}: B^{1} \rightarrow \mathcal{M}$ that extends $\phi^{0}$ by Proposition 4.6. Hence there is a Lipschitz map $\Delta^{1} \rightarrow \mathcal{M}$ for all edges $\Delta^{1}$ of the triangulation $K$ that meet the complex $T^{m}$ of $\mathcal{M}$ at one or both vertices but do not lie in $T^{m}$.

For faces $\Delta^{2}$ of the triangulation $K$ with at least one edge or vertex contained in the complex $T^{m}$ of $\mathcal{M}$ such that the face does not lie in $T^{m}$, we can use the previous step: by noting the edges of such a face $\Delta^{2}$ can be mapped into $\mathcal{M}$ using $\pi_{0}(\mathcal{M})=0$, and as $\partial \Delta^{2} \simeq S^{1}$ in a bi-Lipschitz fashion, we have a Lipschitz mapping $\phi^{1}: S^{1} \rightarrow \mathcal{M}$. Since $\pi_{1}(\mathcal{M})=0$ there exists a Lipschitz extension $\Phi^{1}: B^{2} \rightarrow \mathcal{M}$ by Proposition 4.6. Hence we can map $\Delta^{2} \rightarrow \mathcal{M}$ for all faces $\Delta^{2}$ of the triangulation $K$ with at least one edge or vertex contained in the complex $T^{m}$. Furthermore, for faces $\Delta^{2}$ that not meet $T^{m}$ at all, we can extend $\partial \Delta^{2} \rightarrow \mathcal{M}$ into the interior by 'edge extensions', that is if the $\mathcal{M}$-values along one edge, or two edges, are known then it is possible to extend the edge values to the interior of the face by lines of constant value. ${ }^{7}$ As faces with common edges will share the same values we have a Lipschitz map $K^{2} \cup T^{m} \rightarrow \mathcal{M}$ for the 2-dimensional skeleton $K^{2}$.

Proceeding inductively, suppose we now have a Lipschitz map $K^{j} \cup T^{m} \rightarrow$ $\mathcal{M}$ for the $j$-dimensional skeleton $K^{j}$ with integer $2 \leqq j \leqq \ell$. Then for any $(j+1)$-simplex $\Delta^{j+1}$ with at least one $j$-dimensional face or lower contained in

7 For instance if one considers a face $\Delta^{2}$ in the 2-plane with vertices $(-1,0),(1,0)$ and $(0,1)$ such that a map $\phi: E_{1} \cup E_{2} \rightarrow \mathcal{M}$ is given along the edges $E_{1}=\langle(-1,0),(0,1)\rangle$ and $E_{2}=\langle(1,0),(0,1)\rangle$, we can extend $\phi$ by 'edge extension' alone the lines $L=\{(x, y)$ : $0 \leqq y \leqq 1-\operatorname{sgn}(x) x\},-1 \leqq x \leqq 1$, to a map $\Delta^{2} \ni(x, y) \mapsto \phi(x, 1-\operatorname{sgn}(x) x)$ that takes the values along the given edges. 
the complex $T^{m}$ of $\mathcal{M}$ such that the simplex is not contained in $T^{m}$, we can use the induction hypothesis, $\partial \Delta^{j+1} \simeq S^{j}$ and the fact that $\pi_{0}(\mathcal{M})=\cdots=\pi_{j-1}(\mathcal{M})=$ 0 to obtain a Lipschitz map $\phi^{j}: S^{j} \rightarrow \mathcal{M}$. As $\pi_{j}(\mathcal{M})=0$ it follows that

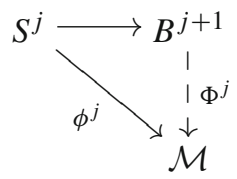

can be completed to a commutative diagram by Proposition 4.6. Hence we can map $\Delta^{j+1} \rightarrow \mathcal{M}$ for all $(j+1)$-simplexes $\Delta^{j+1}$ that meet $T^{m}$ with at least one $j$-dimensional face or lower contained in $T^{m}$. Then as before, for any $(j+1)$ simplexes $\Delta^{j+1}$ that do not meet $T^{m}$ at all, we can extend $\partial \Delta^{j+1} \rightarrow \mathcal{M}$ into the interior by ' $j$-face extensions'. Hence we have a Lipschitz map $K^{j+1} \cup T^{m} \rightarrow \mathcal{M}$ for the $(j+1)$-dimensional complex $K^{j+1}$.

As the obstruction of $\pi_{\ell+1}(\mathcal{M}) \neq 0$, we then have a Lipschitz retraction

$$
\phi: K^{\ell+1} \cup T^{m} \rightarrow \mathcal{M}
$$

by the above construction. Furthermore, by taking a 'cubication' subcomplex $K^{\prime}$ of $K$ if necessary, Lemma 4.4 yields a locally Lipschitz retraction

$$
\psi:\left(K^{k} \backslash L^{k-\ell-2}\right) \cup T^{m} \rightarrow K^{\ell+1} \cup T^{m}
$$

such that $|D \psi(x)| \leqq C / \operatorname{dist}\left(x, L^{k-\ell-2}\right)$ for every $x \in\left(K^{k} \backslash L^{k-\ell-2}\right) \cup T^{m}$. The desired conclusion now follows by setting $X=F\left(\overline{L^{k-\ell-2} \backslash T^{m}}\right)$ with the locally Lipschitz retraction $\Theta=\phi \circ \psi \circ F^{-1}$.

\subsection{An Extension Result for Sobolev Mappings}

From the trace map $\Re: W^{1, p}\left(\Omega, \mathbb{R}^{N}\right) \rightarrow W^{1-\frac{1}{p}, p}\left(\partial \Omega, \mathbb{R}^{N}\right)$ on a Lipschitz domain $\Omega \subset \mathbb{R}^{n}$, any map in $W^{1, p}(\Omega, \mathcal{M})$ has a trace in $W^{1-\frac{1}{p}, p}(\partial \Omega, \mathcal{M})$ by Gagliardo's theorem. We ask: under what conditions can any given map in $W^{1-\frac{1}{p}, p}(\partial \Omega, \mathcal{M})$ be the trace of a map in $W^{1, p}(\Omega, \mathcal{M})$ ? When $1<p<n$ the condition $\pi_{\lfloor p\rfloor-1}(\mathcal{M})=0$ is necessary for Sobolev extensions to exist by a result of [10, Theor. 4]. A recent paper of [7] has shown the condition of $\ell$-connectedness of $\mathcal{M}$ for $1<p<\ell+2$ (with $p<n$ ) is both necessary and sufficient for the Sobolev extension problem. For the purposes here we appeal to a construction in [53, Lem. A.1] (when the target manifold is $S^{2}$ ), and the generalisation given in [56, Lem. 6.2], to prove the following:

Lemma 4.7. [56] Let $\Omega \subset \mathbb{R}^{n}$ be a Lipschitz domain and $\widetilde{\mathcal{M}} \subset \mathbb{R}^{k}, k \geqq 3$, be a compact $\ell$-connected submanifold for some integer $1 \leqq \ell \leqq k-2$. Let $\phi \in\left(W^{1, p} \cap L^{\infty}\right)\left(\Omega, \mathbb{R}^{k}\right)$ for $1<p<\ell+2$ be such that the trace $\mathfrak{R} \phi \in$ $W^{1-\frac{1}{p}, p}(\partial \Omega, \widetilde{\mathcal{M}})$. Then there exists $\psi \in\left(\phi+W_{0}^{1, p}\left(\Omega, \mathbb{R}^{k}\right)\right) \cap W^{1, p}(\Omega, \widetilde{\mathcal{M}})$ and a constant $C=C(n, p, k, \ell, \widetilde{\mathcal{M}})>0$ such that

$$
\int_{\Omega}|D \psi|^{p} \leqq C \int_{\Omega}|D \phi|^{p}, \quad \int_{\Omega}|V(D \psi)|^{2} \leqq C \int_{\Omega}|V(D \phi)|^{2}
$$


and

$$
\int_{\Omega}|x-y|^{1-n}|D \psi(y)| \mathrm{d} y \leqq C \int_{\Omega}|x-y|^{1-n}|D \phi(y)| \mathrm{d} y
$$

for almost everywhere $x \in \Omega$.

Proof. Let $U=\left\{\xi \in \mathbb{R}^{k}: \operatorname{dist}(\xi, \widetilde{\mathcal{M}})<\rho\right\}$, for some $0<\rho<\operatorname{reach}(\widetilde{\mathcal{M}})$, be a neighbourhood with the unique nearest point property. By Theorem 2.4 the map $\Pi: \bar{U} \rightarrow \widetilde{\mathcal{M}}$, associating to each $\xi \in \bar{U}$ the unique $\xi_{0} \in \widetilde{\mathcal{M}} \operatorname{such}$ that $\operatorname{dist}(\xi, \widetilde{\mathcal{M}})=$ $\left|\xi-\xi_{0}\right|$, is Lipschitz continuous. We observe that $\bar{U}$ and $\widetilde{\mathcal{M}}$ are homotopy equivalent spaces, since there exists a strong deformation retraction $h_{t}(\xi)=(1-t) \xi+t \Pi(\xi)$ for $\xi \in \bar{U}$ and $0 \leqq t \leqq 1$. Hence we have $\pi_{j}(\bar{U})=\pi_{j}(\overline{\mathcal{M}})=0$ for $j=0, \ldots, \ell$ (cf. [49, Prop. 1.17]).

As $\widetilde{\mathcal{M}}$ is compact and $\phi \in L^{\infty}$ there exists a $k$-dimensional cube $Q$ such that $\widetilde{\mathcal{M}} \subset \bar{U} \subset Q$ and $\operatorname{dist}(\phi, \widetilde{\mathcal{M}}) \leqq \frac{1}{2} \operatorname{dist}(\widetilde{\mathcal{M}}, \partial Q)$ almost everywhere. By Lemma 4.5 there is a locally Lipschitz retraction $\Theta: Q \backslash X \rightarrow \bar{U}$ for some $(k-$ $\ell-2$ )-dimensional Lipschitz polyhedron $X \subset Q \backslash \bar{U}$ (note that the construction assures $X$ is strictly away from $\widetilde{\mathcal{M}}$ ). Thus we have a mapping

$$
P=\Pi \circ \Theta: Q \backslash X \rightarrow \widetilde{\mathcal{M}}
$$

which, by Lemma 4.5 , has

$$
|\nabla P(\xi)| \leqq \frac{C}{\operatorname{dist}(\xi, X)}
$$

Also, by a change of variables and the definition of the dual skeleton, there exists a positive constant $C=C(p, k, \ell, \widetilde{\mathcal{M}})$ such that

$$
\int_{Q} \frac{d \xi}{\operatorname{dist}(\xi, X)^{p}} \leqq C<+\infty
$$

since $\operatorname{dim} X \leqq k-\ell-2$ and $1<p<\ell+2$.

Now for a sufficiently small $0<\sigma<\min \left\{\frac{\rho}{2}, \frac{1}{2} \operatorname{dist}(\widetilde{\mathcal{M}}, \partial Q)\right\}$ and a point $a \in B_{\sigma}^{k}=\left\{\xi \in \mathbb{R}^{k}:|\xi|<\sigma\right\}$, denote the translations $Q_{a}=\{\xi+a: \xi \in Q\}$ and $X_{a}=\{\xi+a: \xi \in X\}$ so that one has the retraction $P_{a}: Q_{a} \backslash X_{a} \rightarrow \overline{\mathcal{M}}$ given by $P_{a}(\xi)=P(\xi-a)$. Then by Fubini's theorem and (15) there exists some $C=C(p, k, \ell)>0$ such that

$$
\begin{aligned}
\int_{B_{\sigma}^{k}} \int_{\Omega}\left|D\left(P_{a} \circ \phi\right)(x)\right|^{p} \mathrm{~d} x d a & \leqq \int_{\Omega}|D \phi(x)|^{p}\left(\int_{B_{\sigma}^{k}}|\nabla P(\phi(x)-a)|^{p} d a\right) \mathrm{d} x \\
& \leqq \int_{\Omega}|D \phi(x)|^{p}\left(\int_{Q}|\nabla P(\xi)|^{p} d \xi\right) \mathrm{d} x \\
& \leqq C \int_{\Omega}|D \phi|^{p}
\end{aligned}
$$


and as $|\xi|^{2},|\xi|^{p} \leqq|V(\xi)|^{2} \leqq c\left(|\xi|^{2}+|\xi|^{p}\right)$, since $p \geqq 2$, we also have

$$
\begin{aligned}
\int_{B_{\sigma}^{k}} \int_{\Omega}\left|V\left(D\left(P_{a} \circ \phi\right)\right)\right|^{2} & \leqq C \int_{B_{\sigma}^{k}} \int_{\Omega}\left(\left|\nabla P_{a}(\phi)\right|^{2}|D \phi|^{2}+\left|\nabla P_{a}(\phi)\right|^{p}|D \phi|^{p}\right) \\
& \leqq \int_{\Omega}|V(D \phi(x))|^{2}\left(\int_{Q}\left(|\nabla P(\xi)|^{2}+|\nabla P(\xi)|^{p}\right) d \xi\right) \mathrm{d} x \\
& \leqq C \int_{\Omega}|V(D \phi)|^{2},
\end{aligned}
$$

and likewise

$$
\begin{aligned}
\int_{B_{\sigma}^{k}} & \int_{\Omega}|x-y|^{1-n}\left|D\left(P_{a} \circ \phi\right)(y)\right| \mathrm{d} y d a \\
& \leqq \int_{\Omega}|x-y|^{1-n}|D \phi(y)|\left(\int_{B_{\sigma}^{k}}|\nabla P(\phi(y)-a)| d a\right) \mathrm{d} y \\
& \leqq \int_{\Omega}|x-y|^{1-n}|D \phi(y)|\left(\int_{Q}|\nabla P(\xi)| d \xi\right) \mathrm{d} y \\
& \leqq C \int_{\Omega}|x-y|^{1-n}|D \phi(y)| \mathrm{d} y
\end{aligned}
$$

for almost everywhere $x \in \Omega$. Furthermore, for some $K>0$ we have

$$
\begin{gathered}
\int_{\Omega}\left|D\left(P_{a} \circ \phi\right)\right|^{p} \mathrm{~d} x \leqq \frac{K}{\left|B_{\sigma}^{k}\right|} \int_{B_{\sigma}^{k}} \int_{\Omega}\left|D\left(P_{b} \circ \phi\right)\right|^{p} \mathrm{~d} x d b \\
\int_{\Omega}\left|V\left(D\left(P_{a} \circ \phi\right)\right)\right|^{2} \mathrm{~d} x \leqq \frac{K}{\left|B_{\sigma}^{k}\right|} \int_{B_{\sigma}^{k}} \int_{\Omega}\left|V\left(D\left(P_{b} \circ \phi\right)\right)\right|^{2} \mathrm{~d} x d b
\end{gathered}
$$

and

$\int_{\Omega}|x-y|^{1-n}\left|D\left(P_{a} \circ \phi\right)(y)\right| \mathrm{d} y \leqq \frac{K}{\left|B_{\sigma}^{k}\right|} \int_{B_{\sigma}^{k}} \int_{\Omega}|x-y|^{1-n}\left|D\left(P_{b} \circ \phi\right)(y)\right| \mathrm{d} y d b$

for a 'good set' of $a \in B_{\sigma}^{k}$ (if not then Markov's inequality implies the inequalities fails with an exceptional set less than or equal to $K^{-1}\left|B_{\sigma}^{k}\right|$ ). Therefore there exists an $a_{0}$ with $\left|a_{0}\right| \leqq \sigma$ such that

$$
\int_{\Omega}\left|D\left(P_{a_{0}} \circ \phi\right)\right|^{p} \leqq \frac{C}{\sigma^{n}} \int_{\Omega}|D \phi|^{p}, \quad \int_{\Omega}\left|V\left(D\left(P_{a_{0}} \circ \phi\right)\right)\right|^{2} \leqq \frac{C}{\sigma^{n}} \int_{\Omega}|V(D \phi)|^{2}
$$

and

$$
\int_{\Omega}|x-y|^{1-n}\left|D\left(P_{a_{0}} \circ \phi\right)(y)\right| \mathrm{d} y \leqq \frac{C}{\sigma^{n}} \int_{\Omega}|x-y|^{1-n}|D \phi(y)| \mathrm{d} y .
$$

for almost everywhere $x \in \Omega$.

By the chain rule and (14) we have $\operatorname{Lip}\left(P_{a} \mid \widetilde{M}\right) \leqq C$, for some $C>0$ independent of $a$, provided $\sigma$ is sufficiently small. Furthermore, as the dimensions of the domain and codomain for $\left.P_{a}\right|_{\widetilde{\mathcal{M}}}$ are equal and $\left.P\right|_{\widetilde{\mathcal{M}}}$ is equal to the identity map 
on $\widetilde{\mathcal{M}}$, the inverse function theorem implies that $\sup _{a \in B_{\sigma}^{k}} \operatorname{Lip}\left(\left.P_{a}\right|_{\widetilde{\mathcal{M}}}\right)^{-1}$ is finite for sufficiently small $\sigma$. By setting

$$
\psi=\left(P_{a_{0}} \mid \widetilde{\mathcal{M}}\right)^{-1} \circ P_{a_{0}} \circ \phi \in W^{1, p}(\Omega, \widetilde{\mathcal{M}})
$$

the desired conclusion follows.

Remark 4.8. One can consider the model case for the above construction by setting $\widetilde{\mathcal{M}}=S^{k-1}$ with $k>2$ [note that $(k-1)$-sphere is $(k-2)$-connected]. In this spherical target case the above construction can in fact be explicitly carried out as follows (cf. [53]). Firstly, let $P_{a}: \mathbb{R}^{k} \backslash\{a\} \rightarrow S^{k-1}$ send $\xi \mapsto \frac{\xi-a}{|\xi-a|}$. As the gradient $D\left(P_{a} \circ \phi\right)(x)=\left(I-\frac{\phi-a}{|\phi-a|} \otimes \frac{\phi-a}{|\phi-a|}\right) \frac{D \phi}{|\phi-a|}$ we have $\left|D\left(P_{a} \circ \phi\right)\right| \leqq C|\phi-a|^{-1}|D \phi|$ for some $C=C(k)>0$. By Fubini's theorem

$$
\begin{aligned}
\int_{B_{\frac{1}{2}}(0)} \int_{\Omega}\left|D\left(P_{a} \circ \phi\right)\right|^{p} \mathrm{~d} x d a & \leqq C \int_{\Omega} \int_{B_{\frac{1}{2}}(0)}|\phi-a|^{-p}|D \phi|^{p} d a \mathrm{~d} x \\
& \leqq C \int_{\Omega}|D \phi|^{p}
\end{aligned}
$$

since there exists some $C=C(k, p)>0$ such that

$$
\int_{B_{\frac{1}{2}}(0)}|\phi-a|^{-p} d a \leqq C<+\infty
$$

provided $1<p<k$. Therefore, by Markov's inequality there exists an $a_{0}$ with $\left|a_{0}\right| \leqq \frac{1}{2}$ such that

$$
\int_{\Omega}\left|D\left(P_{a_{0}} \circ \phi\right)\right|^{p} \leqq C \int_{\Omega}|D \phi|^{p} .
$$

Now consider the map $\left(\left.P_{a}\right|_{S^{k-1}}\right)^{-1}: S^{k-1} \rightarrow S^{k-1}$, for any $|a| \leqq \frac{1}{2}$, which sends

$$
\eta \mapsto a+\left[-a \cdot \eta+\left((a \cdot \eta)^{2}+1-|a|^{2}\right)^{\frac{1}{2}}\right] \eta
$$

Since $\left|D\left(\left.P_{a}\right|_{S^{k-1}}\right)^{-1}(\eta)\right| \leqq C$ uniformly independent of $a$, the desired conclusion follows by setting $\psi=\left(\left.P_{a_{0}}\right|_{S^{k-1}}\right)^{-1} \circ P_{a_{0}} \circ \phi \in W^{1, p}\left(\Omega, S^{k-1}\right)$.

\subsection{A Hole-Filling Comparison Map Construction}

We can construct the following suitable comparison maps by appealing to the lifting result of Theorem 4.1 together with the extension result of Lemma 4.7. Note that the connectedness assumption on the covering space is deemed canonical as a covering space is a universal covering space if it is simply connected. 
Lemma 4.9. Let $\mathcal{M} \subset \mathbb{R}^{N}$ be a connected compact submanifold without boundary and $\pi: \widetilde{\mathcal{M}} \rightarrow \mathcal{M}$ be its universal cover such that $\widetilde{\mathcal{M}}$ is compact and $\ell$-connected for some integer $\ell \geqq 1$. If $u \in W^{1, p}(\Omega, \mathcal{M})$ for $2 \leqq p<\ell+2$ on a Lipschitz domain $\Omega \subset \mathbb{R}^{n}$, then on any ball $B_{s} \subset \subset \Omega$ there exists $v \in\left(u+W_{0}^{1, p}\left(B_{s}, \mathbb{R}^{N}\right)\right) \cap$ $W^{1, p}\left(B_{s}, \mathcal{M}\right)$ and a constant $C=C(n, N, p, k, \ell)>0$ such that for any $0<r<$ $s$,

$$
\begin{gathered}
\int_{B_{s}}|D v|^{p} \leqq C \int_{B_{s} \backslash B_{r}}|D u|^{p}+C \int_{B_{s}}\left|\frac{\varphi-(\varphi)_{B_{s}}}{s-r}\right|^{p} \\
\int_{B_{s}}|V(D v)|^{2} \leqq C \int_{B_{s} \backslash B_{r}}|V(D u)|^{2}+C \int_{B_{s}}\left|V\left(\frac{\varphi-(\varphi)_{B_{s}}}{s-r}\right)\right|^{2}
\end{gathered}
$$

and for almost everywhere $x \in B_{s}$,

$$
|u(x)-v(x)| \leqq C \int_{B_{s}}|x-y|^{1-n}\left(|D u(y)|+\left|\frac{\varphi(y)-(\varphi)_{B_{s}}}{s-r}\right|\right) \mathrm{d} y
$$

where $\varphi \in W^{1, p}\left(B_{s}, \widetilde{\mathcal{M}}\right)$ is such that $u=\pi \circ \varphi$.

Proof. Suppose $\widetilde{\mathcal{M}} \subset \mathbb{R}^{k}$ for some $k \geqq 3$ without loss of generality. For any $u \in W^{1, p}(\Omega, \mathcal{M}), p \geqq 2$, there exists $\varphi \in W^{1, p}(\Omega, \widetilde{\mathcal{M}})$ such that $u=\pi \circ \varphi$ and $|D u|=|D \varphi|$ almost everywhere by Theorem 4.1. For any $B_{s} \subset \subset \Omega$ and $0<r<s$ let $\eta \in C_{c}^{\infty}(\Omega)$ be a cut-off function such that $\eta=1$ on $B_{r}, \eta=0$ on $\Omega \backslash B_{S}, 0 \leqq \eta \leqq 1$ and $|D \eta| \leqq C /(s-r)$. By setting

$$
\phi=(1-\eta) \varphi+\eta(\varphi)_{B_{s}}
$$

we have $\phi \in\left(W^{1, p} \cap L^{\infty}\right)\left(B_{s}, \mathbb{R}^{k}\right)$ with $\mathfrak{R} \phi=\mathfrak{R} \varphi \in W^{1-\frac{1}{p}, p}\left(\partial B_{s}, \widetilde{\mathcal{M}}\right)$. So by Lemma 4.7 there exists $\psi \in\left(\varphi+W_{0}^{1, p}\left(B_{S}, \mathbb{R}^{k}\right)\right) \cap W^{1, p}\left(B_{S}, \widetilde{\mathcal{M}}\right)$ such that

$$
\int_{B_{s}}|D \psi|^{p} \leqq C \int_{B_{s}}\left|(1-\eta) D \varphi-D \eta \otimes\left(\varphi-(\varphi)_{B_{s}}\right)\right|^{p},
$$

and, by (11c), we also have that

$$
\int_{B_{s}}|V(D \psi)|^{2} \leqq C \int_{B_{s} \backslash B_{r}}|V(D \varphi)|^{2}+C \int_{B_{s}}\left|V\left(D \eta \otimes\left(\varphi-(\varphi)_{B_{s}}\right)\right)\right|^{2} .
$$

Then by defining $v=\pi \circ \psi$ we have $\Re v=\pi \circ \Re \varphi=\Re u$. The desired result now follows since $|D v|=|D \psi|$ almost everywhere and by standard potential estimates (cf. [47, (7.37)])

$$
|u(x)-v(x)| \leqq C \int_{B_{s}}|x-y|^{1-n}|D u(y)-D v(y)| \mathrm{d} y .
$$




\section{Higher Integrability}

Given the results of Section 4 we obtain Caccioppoli inequalities of the first kind using only the growth (h1), (3) and the coercivity (h4). An application of Gehring's lemma then implies local higher integrability for the gradient $D u$.

Lemma 5.1. Let $\mathcal{M}$ be a connected compact manifold without boundary and $\pi: \widetilde{\mathcal{M}} \rightarrow \mathcal{M}$ be its universal cover such that $\widetilde{\mathcal{M}}$ is compact and $\ell$-connected for some integer $\ell \geqq 1$. Suppose the integrand $f$ satisfies $(h 1),(h 4)$ and $(3)$ for $2 \leqq p<\ell+2$. Then for any holonomic $\mathfrak{F}$-minimiser $u \in W_{\text {loc }}^{1, p}(\Omega, \mathcal{M})$ there exist $C>0$ and $q>p$ independent of $u$ such that $D u \in L_{\mathrm{loc}}^{q}\left(\Omega, \mathbb{R}^{N n}\right)$ and for any $B_{R}\left(x_{0}\right) \subset \subset \Omega$,

$$
\left(f_{B_{R / 2}\left(x_{0}\right)}|D u|^{q}\right)^{1 / q} \leqq C\left(f_{B_{R}\left(x_{0}\right)} 1+|D u|^{p}\right)^{1 / p} .
$$

Proof. For $B_{R}\left(x_{0}\right) \subset \subset \Omega$ let $\frac{R}{2} \leqq r<s \leqq R$ and choose a cut-off function $\eta \in C_{c}^{\infty}(\Omega)$ such that $\eta=1$ on $B_{r}\left(x_{0}\right), \eta=0$ on $\Omega \backslash B_{s}\left(x_{0}\right), 0 \leqq \eta \leqq 1$ and $|D \eta| \leqq C /(s-r)$. Define $\phi_{1}=\eta\left(u-(u)_{x_{0}, R}\right)$ and $\phi_{2}=(1-\eta)\left(u-(u)_{x_{0}, R}\right)$. Using (h4) we get

$$
\begin{aligned}
\int_{B_{s}} \gamma\left|D \phi_{1}\right|^{p}+\psi(0) & \leqq \int_{B_{s}} \psi\left(D \phi_{1}\right) \leqq \int_{B_{s}} f\left(x, u, D \phi_{1}\right) \\
& =\int_{B_{s}} f\left(x, u, D u-D \phi_{2}\right) .
\end{aligned}
$$

From (3) we estimate

$$
\begin{aligned}
\int_{B_{s}} f\left(x, u, D u-D \phi_{2}\right)-f(x, u, D u) & \leqq C \int_{B_{s}}\left(1+|D u|^{p-1}+\left|D \phi_{2}\right|^{p-1}\right)\left|D \phi_{2}\right| \\
& \leqq C \int_{B_{s} \backslash B_{r}} 1+|D u|^{p}+\left|\frac{u-(u)_{R}}{s-r}\right|^{p},
\end{aligned}
$$

since $D \phi_{2}=(1-\eta) D u-\left(u-(u)_{R}\right) \otimes D \eta$. Also, by the minimality of $u$ we have

$$
\int_{B_{s}} f(x, u, D u) \leqq \int_{B_{s}} f(x, v, D v)
$$

for any $v \in\left(u+W_{0}^{1, p}\left(B_{S}, \mathbb{R}^{N}\right)\right) \cap W^{1, p}\left(B_{S}, \mathcal{M}\right)$. Now by the lifting result of Theorem 4.1 there exists $\varphi \in W^{1, p}\left(B_{s}, \widetilde{\mathcal{M}}\right)$ such that $u=\pi \circ \varphi$ with $|D \varphi|=$ $|D u|$ almost everywhere. Then by Lemma 4.9 one has a suitable competitor $v \in$ $\left(u+W^{1, p}\left(B_{s}, \mathbb{R}^{N}\right)\right) \cap W^{1, p}\left(B_{S}, \mathcal{M}\right)$ such that

$$
\begin{aligned}
\int_{B_{s}}|D v|^{p} & \leqq C \int_{B_{s} \backslash B_{r}}|D u|^{p}+C \int_{B_{s}}\left|\frac{\varphi-(\varphi)_{R}}{s-r}\right|^{p} \\
& \leqq C \int_{B_{s} \backslash B_{r}}|D u|^{p}+C \frac{R^{n+p}}{(s-r)^{p}}\left(f_{B_{R}}|D u|^{p_{*}}\right)^{p / p_{*}}
\end{aligned}
$$


by the Sobelov-Poincaré inequality with $p_{*}=\frac{n p}{n+p}$. Then by (16) and (h1) we obtain

$$
\begin{aligned}
\int_{B_{r}}|D u|^{p} \leqq \int_{B_{s}}\left|D \phi_{1}\right|^{p} \leqq & \frac{1}{\gamma} \int_{B_{s}}\left(f\left(x, u, D u-D \phi_{2}\right)+|\psi(0)|\right) \\
\leqq & C R^{n}+C \int_{B_{s} \backslash B_{r}}|D u|^{p} \\
& +C \frac{R^{n+p}}{(s-r)^{p}}\left(f_{B_{R}}|D u|^{p_{*}}\right)^{p / p_{*}}
\end{aligned}
$$

So by Widman's hole filling trick we have

$$
\int_{B_{r}}|D u|^{p} \leqq \chi \int_{B_{s}}|D u|^{p}+R^{n}+\frac{R^{n+p}}{(s-r)^{p}}\left(f_{B_{R}}|D u|^{p_{*}}\right)^{p / p_{*}}
$$

where $\chi=\frac{C}{1+C}<1$. Then by the standard iteration of [40, Lem. 1.1] we conclude

$$
f_{B_{R / 2}\left(x_{0}\right)}|D u|^{p} \leqq C+C\left(f_{B_{R}\left(x_{0}\right)}|D u|^{p_{*}}\right)^{p / p_{*}}
$$

for all $B_{R}\left(x_{0}\right) \subset \Omega$. In which case by Gehring's lemma (cf. [45, Prop. 5.1]) there exists $q>p$ such that

$$
\left(f_{B_{R / 2}}|D u|^{q}\right)^{1 / q} \leqq C\left(f_{B_{R}} 1+|D u|^{p}\right)^{1 / p}
$$

for all $B_{R} \subset \subset \Omega$. In particular, $D u \in L_{\text {loc }}^{q}\left(\Omega, \mathbb{R}^{N n}\right)$.

We can also get higher integrability up to the boundary by adapting the proof of the previous lemma using standard up to the boundary arguments (cf. [5, Lem. 3.5], [42, p. 152]) together with a suitable up to the boundary version of Lemma 4.9.

Lemma 5.2. Let $\mathcal{M}$ be a connected compact manifold without boundary and $\pi: \widetilde{\mathcal{M}} \rightarrow \mathcal{M}$ be its universal cover such that $\widetilde{\mathcal{M}}$ is compact and $\ell$-connected for some integer $\ell \geqq 1$. Suppose the integrand $f$ satisfies $(h 1),(h 4)$ and (3) for $2 \leqq p<\ell+2$. Fix $\left(x_{0}, u_{0}\right) \in \Omega \times \mathbb{R}^{N}$ and on a given ball $\mathrm{B} \subset \Omega$ assume $u \in W^{1, q_{0}}(\mathrm{~B}, \mathcal{M})$ for some $q_{0}>p$. If $\widehat{u} \in\left(u+W_{0}^{1, p}\left(\mathrm{~B}, \mathbb{R}^{N}\right)\right) \cap W^{1, p}(\mathrm{~B}, \mathcal{M})$ satisfies

$$
\int_{\mathrm{B}} f\left(x_{0}, u_{0}, D \widehat{u}\right) \leqq \int_{\mathrm{B}} f\left(x_{0}, u_{0}, D v\right)
$$

for all $v \in\left(u+W_{0}^{1, p}\left(\mathrm{~B}, \mathbb{R}^{N}\right)\right) \cap W^{1, p}(\mathrm{~B}, \mathcal{M})$, then there exists $C>0, q \in\left(p, q_{0}\right]$ such that $\widehat{u} \in W^{1, q}(\mathrm{~B}, \mathcal{M})$ and

$$
\left(f_{\mathrm{B}}|D \widehat{u}|^{q}\right)^{1 / q} \leqq C\left(f_{\mathrm{B}}|D \widehat{u}|^{p}\right)^{1 / p}+C\left(f_{\mathrm{B}} 1+|D u|^{q_{0}}\right)^{1 / q_{0}} .
$$




\section{An Application of Ekeland's Variational Principle}

In this section we use the following lemma of Ekeland together with ideas from ACERBI and FUSCO [3] to show an holonomic $\mathfrak{F}$-minimiser almost minimises the so-called frozen problem. This result is then subsequently utilised in Section 8 to show approximate tangential harmonicity via a linearisation strategy applied to the frozen integrand.

Lemma 6.1. [31] Let $(X, d)$ be a complete metric space and $\mathcal{J}: X \rightarrow \mathbb{R} \cup\{+\infty\}$ be a lower semicontinuous functional that is bounded from below but not identically $+\infty$. For $\varepsilon>0$ and a point $u \in X$ such that

$$
\mathcal{J}(u)<\varepsilon+\inf \mathcal{J}
$$

there exists some point $v \in X$ such that $d(u, v) \leqq 1$ and

$$
\mathcal{J}(v) \leqq \mathcal{J}(w)+\varepsilon d(v, w)
$$

for every $w \in X$.

To satisfy the lower semicontinuity requirement we recall the following:

Lemma 6.2. [3, IV.6] Let the integrand $f$ satisfy (h1) and (h2). If $B_{R} \subset \Omega$ is a ball, $\left(x_{0}, u_{0}\right) \in \Omega \times \mathbb{R}^{N}$ and $u \in W^{1, p}\left(B_{R}, \mathcal{M}\right)$ the functional

$$
w \mapsto \int_{B_{R}} f\left(x_{0}, u_{0}, D w\right)
$$

is sequentially weakly lower semicontinuous on $\left(u+W_{0}^{1, p}\left(B_{R}, \mathbb{R}^{N}\right)\right) \cap W^{1, p}\left(B_{R}, \mathcal{M}\right)$ and satisfies

$$
\int_{B_{R}} f\left(x_{0}, u_{0}, D w\right) \geqq \gamma \int_{B_{R}}|D w|^{p}-c \int_{B_{R}}\left(1+|D u|^{p}\right) .
$$

Now for a given $u \in W_{\text {loc }}^{1, p}(\Omega, \mathcal{M})$ and a fixed ball $B_{2 R}\left(x_{0}\right) \subset \subset \Omega$ with $0<R<1$, suppose $\widehat{u} \in\left(u+W_{0}^{1, p}\left(B_{R}\left(x_{0}\right), \mathbb{R}^{N}\right)\right) \cap W^{1, p}\left(B_{R}\left(x_{0}\right), \mathcal{M}\right)$ is a minimiser of the the corresponding frozen problem:

$$
\int_{B_{R}\left(x_{0}\right)} f\left(x_{0},(u)_{x_{0}, R}, D \widehat{u}\right) \leqq \int_{B_{R}\left(x_{0}\right)} f\left(x_{0},(u)_{x_{0}, R}, D w\right)
$$

for all $w \in\left(u+W_{0}^{1, p}\left(B_{R}\left(x_{0}\right), \mathbb{R}^{N}\right)\right) \cap W^{1, p}\left(B_{R}\left(x_{0}\right), \mathcal{M}\right)$. Note that the existence of $\widehat{u}$ is obtained by direct methods whereby the Rellich-Kondrachov theorem assures the limit $\widehat{u}$ of the minimising subsequence satisfies the condition $\widehat{u} \in \mathcal{M}$ almost everywhere.

By defining

$$
\mathcal{J}(w)=f_{B_{R}\left(x_{0}\right)} f\left(x_{0},(u)_{x_{0}, R}, D w\right) \mathrm{d} x,
$$

we see (following the proof of [3, Lem. IV.7] together with Lemmas 5.1, 5.2, (h3) and the minimality of $\widehat{u}$ ) that any holonomic $\mathfrak{F}$-minimiser $u$ of the 'full problem' is an almost minimiser of the 'frozen problem' in the following sense: 
Proposition 6.3. If for every $K>0$, under the assumptions of Lemmas 5.1 and 5.2 for an integrand $f$ satisfying $(h 1),(h 2),(h 3)$ and $(h 4)$, the map $u \in W_{\mathrm{loc}}^{1, p}(\Omega, \mathcal{M})$ is an holonomic $\mathfrak{F}$-minimiser with

$$
\left(f_{B_{2 R}\left(x_{0}\right)}|D u|^{p}\right)^{1 / p} \leqq K
$$

on some fixed $B_{2 R}\left(x_{0}\right) \subset \subset \Omega$, then there exists $c_{1}=c_{1}(K)>0$ such that

$$
\mathcal{J}(u) \leqq \mathcal{J}(\widehat{u})+c_{1} R^{\mu\left(1-\frac{p}{q}\right)},
$$

where $\widehat{u} \in\left(u+W_{0}^{1, p}\left(B_{R}\left(x_{0}\right), \mathbb{R}^{N}\right)\right) \cap W^{1, p}\left(B_{R}\left(x_{0}\right), \mathcal{M}\right)$ is a minimiser of $(20)$.

By endowing the space $\left(u+W_{0}^{1, p}\left(B_{R}\left(x_{0}\right), \mathbb{R}^{N}\right)\right) \cap W^{1, p}\left(B_{R}\left(x_{0}\right), \mathcal{M}\right)$ with the metric

$$
d\left(v_{1}, v_{2}\right)=c_{1}(K)^{-1} R^{-\beta}\left(f_{B_{R}\left(x_{0}\right)}\left|D v_{1}-D v_{2}\right|^{p}\right)^{1 / p},
$$

with $\beta=\frac{\mu}{2}\left(1-\frac{p}{q}\right)$, the functional $\mathcal{J}$ is lower semicontinuous (cf. Lemma 6.2) and clearly

$$
\inf \mathcal{J}=\mathcal{J}(\widehat{u})
$$

by definition. So by Lemma 6.1 and the result of Proposition 6.3 there exists $v \in$ $\left(u+W_{0}^{1, p}\left(B_{R}\left(x_{0}\right), \mathbb{R}^{N}\right)\right) \cap W^{1, p}\left(B_{R}\left(x_{0}\right), \mathcal{M}\right)$ satisfying

$$
\left(f_{B_{R}\left(x_{0}\right)}|D v-D u|^{p}\right)^{1 / p} \leqq c_{1}(K) R^{\beta}
$$

which is also a minimiser in the sense that

$$
\mathcal{J}(v) \leqq \mathcal{J}(w)+R^{\beta}\left(f_{B_{R}\left(x_{0}\right)}|D v-D w|^{p}\right)^{1 / p}
$$

among all $w \in\left(u+W_{0}^{1, p}\left(B_{R}\left(x_{0}\right), \mathbb{R}^{N}\right)\right) \cap W^{1, p}\left(B_{R}\left(x_{0}\right), \mathcal{M}\right)$. Moreover, by following the proof of Lemma 3.1 we also obtain the following:

Corollary 6.4. If $v \in\left(u+W_{0}^{1, p}\left(B_{R}\left(x_{0}\right), \mathbb{R}^{N}\right)\right) \cap W^{1, p}\left(B_{R}\left(x_{0}\right), \mathcal{M}\right)$ is a minimiser of (22) we have

$$
\left|f_{B_{R}\left(x_{0}\right)} D_{\xi} f\left(x_{0},(u)_{x_{0}, R}, D v\right) D(\nabla \Pi(v) \phi)\right| \leqq R^{\beta}\left(f_{B_{R}\left(x_{0}\right)}|D(\nabla \Pi(v) \phi)|^{p}\right)^{1 / p}
$$

for all $\phi \in W_{0}^{1, p}\left(B_{R}\left(x_{0}\right), \mathbb{R}^{N}\right) \cap L^{\infty}\left(B_{R}\left(x_{0}\right), \mathbb{R}^{N}\right)$, where $\Pi$ is a retraction from a tubular neighbourhood onto $\mathcal{M}$, provided the integrand $f$ satisfies $(h 1)$. 


\section{The Tangential $\mathbb{A}$-Harmonic Approximation Lemma}

A symmetric bilinear form $\mathbb{A}$ on $\mathbb{R}^{N n}$ is said to be strongly elliptic in the sense of Legendre-Hadamard, or simply elliptic, if for all $\eta \in \mathbb{R}^{N}$ and $\xi \in \mathbb{R}^{n}$ there holds

$$
\mathbb{A}(\eta \otimes \xi, \eta \otimes \xi) \geqq \ell|\eta|^{2}|\xi|^{2}
$$

for some $\ell>0$. We call $\ell$ an ellipticity constant associated to $\mathbb{A}$. Note that $\mathbb{A}$ is bounded [in the sense that $\mathbb{A}\left(\zeta_{1}, \zeta_{2}\right) \leqq L\left|\zeta_{1}\right|\left|\zeta_{2}\right|$ for all $\zeta_{1}, \zeta_{2} \in \mathbb{R}^{N n}$ ] for free (by finite dimensionality). A classical result shows this condition to be equivalent to the strong ellipticity condition

$$
\int_{\Omega} \mathbb{A}(D \phi, D \phi) \geqq \ell \int_{\Omega}|D \phi|^{2}
$$

for all $\phi \in W_{0}^{1,2}\left(\Omega, \mathbb{R}^{N}\right)$.

Looking to the notion of harmonicity used in the limit of indirect blow-up proof of [34, pp. 83-5] we say:

Definition 7.1. A map $h \in W^{1,2}\left(\Omega, \mathbb{R}^{N}\right)$ is a tangential $\mathbb{A}$-harmonic map about a given point $\theta \in \mathcal{M}$ if $\mathbb{A}$ satisfies the strong ellipticity condition (23) and

$$
\int_{\Omega} \mathbb{A}(D h, D \phi)=0
$$

for all $\phi \in C_{c}^{\infty}\left(\Omega, \mathbb{R}^{N}\right)$ such that $\nabla \Pi(\theta) D \phi=D \phi$ and $\nabla \Pi(\theta) D h=D h$ almost everywhere $(\Omega)$.

Here $\Pi$ is the retraction given by (12) and $\nabla \Pi(\theta)$ is a projection onto the tangent space $T_{\theta} \mathcal{M}$ (cf. Corollary 2.5). Hence the condition $\nabla \Pi(\theta) D h=D h$ is equivalent to requiring $D_{i} h \in T_{\theta} \mathcal{M}$ for $i=1, \ldots, n$. Furthermore, we also introduce the map $\mathrm{C}_{\theta}: \mathbb{R}^{N} \rightarrow \theta+T_{\theta} \mathcal{M}$ that sends

$$
\mathrm{C}_{\theta}: v \mapsto \theta+\nabla \Pi(\theta)(v-\theta)
$$

Definition 7.2. A map $u \in W^{1, p}\left(B_{R}, \mathcal{M}\right)$ is a $\delta$-approximate tangential $\mathbb{A}$ harmonic map about a given point $\theta \in \mathcal{M}$ with exponents $p \geqq 2, \alpha>1,0<\beta \leqq 1$ if $\mathbb{A}$ satisfies the strong ellipticity condition (23) and for some $\delta>0$ we have

$$
f_{B_{R}}|V(D u-\nabla \Pi(\theta) D u)|^{2} \leqq \delta^{2} R+\delta^{2\left(1-\frac{1}{\alpha}\right)}\left(f_{B_{R}}\left|V\left(D u-(D u)_{R}\right)\right|^{2 \alpha}\right)^{\frac{1}{\alpha}}
$$

and

$$
\left|f_{B_{R}} \mathbb{A}(D u, \nabla \Pi(\theta) D \phi)\right| \leqq \delta\left(R^{\beta}+f_{B_{R}}\left|V\left(D u-(D u)_{R}\right)\right|^{2}\right)^{\frac{1}{2}}\|D \phi\|_{\infty}
$$

for all $\phi \in C_{c}^{\infty}\left(B_{R}, \mathbb{R}^{N}\right)$. 
The following a priori estimates for tangential $\mathbb{A}$-harmonic maps are obtained by a change of orthonormal basis. This reduces the problem to an unconstrained elliptic system from which the result can be obtained.

Lemma 7.3. (Weyl-type) Let $h \in W^{1,1}\left(\Omega, \mathbb{R}^{N}\right)$ be such that

$$
\int_{\Omega} \mathbb{A}(D h, D \phi)=0
$$

for any $\phi \in C_{c}^{1}\left(\Omega, \mathbb{R}^{N}\right)$ with $\nabla \Pi(\theta) D \phi=D \phi$ and $\nabla \Pi(\theta) D h=$ Dh almost everywhere about a given point $\theta \in \mathcal{M}$, where $\mathbb{A}$ is strongly elliptic with elliptic constant $\ell$ and upper bound $L$. Then $h \in C_{\mathrm{loc}}^{\infty}\left(\Omega, \mathbb{R}^{N}\right)$ and for any $B_{R} \subset \subset \Omega$ we have

$$
\sup _{B_{R / 2}}\left|D^{k} h\right| \leqq \frac{C}{R^{k-1}} f_{B_{R}}|D h|
$$

for some $C=C\left(n, N, k, \frac{L}{\ell}\right)>0$ and any integer $k \geqq 1$.

Proof. As $\nabla \Pi(\theta)$ is a projection onto the tangent space $T_{\theta} \mathcal{M}$ (cf. Corollary 2.5) there exists an $N \times N$ orthogonal matrix $R$ such that

$$
R \nabla \Pi(\theta) R^{T}=I_{m} \oplus 0_{N-m}
$$

where $m=\operatorname{dim} \mathcal{M}$. If $S$ denotes the $N \times N$ matrix on the right-hand side of (24) we find that $D(R h)=S D(R h)$ and $D(R \phi)=S D(R \phi)$, since $\nabla \Pi(\theta) D h=D h$ and $\nabla \Pi(\theta) D \phi=D \phi$ almost everywhere. Thus $(R \phi)_{i}^{a}=(R h)_{i}^{a}=0$ for $a=$ $m+1, \ldots, N$ and $i=1, \ldots, n$. With this one finds that

$$
\begin{aligned}
0=\int_{\Omega}\left(D_{i} h\right)^{T} \cdot \mathbb{A}_{i j} \cdot\left(D_{j} \phi\right) & =\int_{\Omega}\left(D_{i}(R h)\right)^{T} \cdot S^{T} R \mathbb{A}_{i j} R^{T} S \cdot D_{j}(R \phi) \\
& =\int_{\Omega}\left(D_{i}(R h)\right)^{T} \cdot \mathbb{B}_{i j} \cdot\left(D_{j}(R \phi)\right),
\end{aligned}
$$

where $\mathbb{B}_{i j}$ denote the upper left $m \times m$ submatrix of $S^{T} R \mathbb{A}_{i j} R^{T} S$. Likewise,

$$
\int_{\Omega}\left(D_{i}(R \phi)\right)^{T} \cdot \mathbb{B}_{i j} \cdot\left(D_{j}(R \phi)\right) \geqq \ell \int_{\Omega}|D \phi|^{2}=\ell \int_{\Omega}\left(D_{i}(R \phi)\right)^{T} \cdot D_{i}(R \phi) .
$$

Thus $H=\left((R h)^{1}, \ldots,(R h)^{m}\right)$ is $\mathbb{B}$-harmonic in the sense that

$$
\operatorname{div} \mathbb{B}(D H)=0
$$

weakly with $\int_{\Omega} \mathbb{B}(D \varphi, D \varphi) \geqq \ell \int_{\Omega}|D \varphi|^{2}$ for all $\varphi \in C_{c}^{1}\left(\Omega, \mathbb{R}^{m}\right)$. In which case $H$ is a solution to an (unconstrained) linear elliptic system with constant coefficients satisfying the strong ellipticity condition (23). Hence $H \in C_{\mathrm{loc}}^{\infty}$ with the desired interior estimate obtained from [17, pp. 150-1]. 
Remark 7.4. As $\left|D h-(D h)_{r}\right| \leqq 2 r \sup _{B_{r}}\left|D^{2} h\right|$ on $B_{r}$, the above lemma implies $\left|D h-(D h)_{r}\right| \leqq C f_{B_{2 R}}\left|\frac{r}{R} D h\right|$ for $0<r<R$. Since $|V(\cdot)|^{2}$ is a convex function for $p \geqq 2$ it follows that

$$
f_{B_{r}}\left|V\left(D h-(D h)_{r}\right)\right|^{2} \leqq C\left(\frac{r}{R}\right)^{2} f_{B_{2 R}}\left|V\left(D h-(D h)_{2 R}\right)\right|^{2} .
$$

By appealing to the same change of variables argument used in Lemma 7.3 and the $L^{p}$-estimates of CAMPANATo and Stampacchia (cf. [18], [44, §7.1.1]) we also obtain the following:

Lemma 7.5. Let $F \in L^{p}\left(\Omega, \mathbb{R}^{N n}\right)$ such that $\nabla \Pi(\theta) F=F$ almost everywhere for a given point $\theta \in \mathcal{M}$ and $p>1$. If $v \in W_{0}^{1, p}\left(\Omega, \mathbb{R}^{N}\right)$ is a solution to

$$
\int_{\Omega} \mathbb{A}(D v, D \phi)=\int_{\Omega} F \cdot D \phi
$$

for any $\phi \in C_{c}^{\infty}\left(\Omega, \mathbb{R}^{N}\right)$ such that $\nabla \Pi(\theta) D \phi=D \phi$ and $\nabla \Pi(\theta) D v=D v$ almost everywhere, where $\mathbb{A}$ is strongly elliptic bilinear form of constant coefficients, then $D v \in L^{p}\left(\Omega, \mathbb{R}^{N n}\right)$ and

$$
\|D v\|_{L^{p}\left(\Omega, \mathbb{R}^{N n}\right)} \leqq c\|F\|_{L^{p}\left(\Omega, \mathbb{R}^{N n}\right)}
$$

for some $c>0$ independent of $v$ and $F$, provided $\Omega \subset \mathbb{R}^{n}$ is a smooth bounded domain.

Let us also recall the following standard pointwise approximation of Sobolev functions that goes back to a result by [4].

Lemma 7.6. Let $v \in W_{0}^{1, p}(\Omega)$ for $p \geqq 1$ and $\Omega \subset \mathbb{R}^{n}$. Then for every $\lambda>0$ there exists $v_{\lambda} \in W_{0}^{1, \infty}(\Omega)$ with $\left\|v_{\lambda}\right\|_{1, \infty ; \Omega} \leqq \lambda$ and

$$
\begin{aligned}
\left\|v_{\lambda}\right\|_{1, p ; \Omega} & \leqq c\|v\|_{1, p ; \Omega} \\
\left|\left\{v_{\lambda} \neq v\right\}\right| & \leqq c \lambda^{-p} \int_{\Omega}|v|^{p}+|D v|^{p}
\end{aligned}
$$

for some $c=c(n, p)>0$.

We can now give a (direct) proof of the following tangential $\mathbb{A}$-harmonic approximation lemma based on the the general Sobolev-Orlicz space proof of [27,30].

Lemma 7.7. For every $0<\varepsilon<1$ there exists $\delta=\delta(\varepsilon, n, N, p, \alpha, \ell, L)>0$ such that if $u \in W^{1, p}\left(B_{R}, \mathcal{M}\right)$, for $p \geqq 2$ with $0<R<1$, is any $\delta$-approximate tangential $\mathbb{A}$-harmonic map about a point $\theta \in \mathcal{M}$ with exponents $\alpha>1$ and $0<\beta \leqq 1$, where $\mathbb{A}$ is a strongly elliptic bilinear form with ellipticity constant $\ell$ and an upper bound $L$, then the unique tangential $\mathbb{A}$-harmonic map $h \in \mathrm{C}_{\theta}(u)+$ $W_{0}^{1, p}\left(B_{R}, \mathbb{R}^{N}\right)$ about $\theta$ satisfies

$$
\begin{aligned}
f_{B_{R}}|V(D u-D h)|^{2} \leqq & \varepsilon\left[R^{\beta}+\left(f_{B_{R}}\left|V\left(D u-(D u)_{R}\right)\right|^{2 \alpha}\right)^{\frac{1}{\alpha}}\right. \\
& \left.+f_{B_{R}}\left|V\left(D u-(D u)_{R}\right)\right|^{2}+\left(f_{B_{R}}\left|V\left(D u-(D u)_{R}\right)\right|^{2}\right)^{\frac{p}{2}}\right] .
\end{aligned}
$$


Note that the existence and uniqueness of the tangential $\mathbb{A}$-harmonic map follows from the standard linear theory.

Proof. First, using (11a) and (11c), we bound

$$
\begin{aligned}
f_{B_{R}}|V(D u-D h)|^{2} \leqq & c_{0} f_{B_{R}}|V(\nabla \Pi(\theta) D u-D h)|^{2}+|V(D u-\nabla \Pi(\theta) D u)|^{2} \\
\leqq & c_{0} f_{B_{R}}|\nabla \Pi(\theta) D u-D h|^{p}+|\nabla \Pi(\theta) D u-D h|^{2} \\
& +c_{0} f_{B_{R}}|V(D u-\nabla \Pi(\theta) D u)|^{2}
\end{aligned}
$$

for some positive $c_{0}=c_{0}(n, N, p)$.

Set $U=\mathrm{C}_{\theta}(u)-h \in W_{0}^{1, p}\left(B_{R}, \mathbb{R}^{N}\right)$ and $H=|D U|^{p-1} \frac{D U}{|D U|} \in$ $L^{q}\left(B_{R}, \mathbb{R}^{N n}\right)$, where $q=\frac{p}{p-1}$, so that $\nabla \Pi(\theta) H=H$ almost everywhere. Let $v \in W_{0}^{1, q}\left(B_{R}, \mathbb{R}^{N}\right)$ be a solution to

$$
\int_{B_{R}} \mathbb{A}(D v, D \varphi)=\int_{B_{R}} H \cdot D \varphi
$$

for any $\varphi \in W_{0}^{1, p}\left(B_{R}, \mathbb{R}^{N}\right)$ such that $\nabla \Pi(\theta) D \varphi=D \varphi$ and $\nabla \Pi(\theta) D v=D v$ almost everywhere. By Lemma 7.5 we have

$$
\int_{B_{R}}|D v|^{q} \leqq \frac{C_{0}}{q} \int_{B_{R}}|H|^{q}
$$

for some $C_{0}=C_{0}(n, N, q, \ell, L)$. From this $L^{q}$-estimate, Equation (26) and the fact that $U \in W_{0}^{1, p}\left(B_{R}, \mathbb{R}^{N}\right)$ satisfies $\nabla \Pi(\theta) D U=D U$ almost everywhere we have

$$
\begin{aligned}
\frac{1}{p} \int_{B_{R}}|D U|^{p}=\int_{B_{R}} \mathbb{A}(D v, D U)-\frac{1}{q}|H|^{q} & \leqq \int_{B_{R}} \mathbb{A}(D v, D U)-\frac{1}{C_{0}}|D v|^{q} \\
& =\int_{B_{R}} \mathbb{A}(D v, \nabla \Pi(\theta) D u)-\frac{1}{C_{0}}|D v|^{q},
\end{aligned}
$$

since $h$ is a tangential $\mathbb{A}$-harmonic map about $\theta$. Similarly, the strong ellipticity condition (23) also implies

$$
\frac{1}{2} f_{B_{R}}|D U|^{2} \leqq \frac{1}{\ell} f_{B_{R}} \mathbb{A}(D U, \nabla \Pi(\theta) D u)-\frac{1}{2} f_{B_{R}}|D U|^{2},
$$

since $h$ is a tangential $\mathbb{A}$-harmonic map about $\theta$ with $U \in W_{0}^{1,2}\left(B_{R}, \mathbb{R}^{N}\right)$ also.

Now set $A=(D u)_{R}$ and denote $\Lambda^{q}=f_{B_{R}}|D v|^{q}$. By Lemma 7.6 there exists a Lipschitz approximation $v_{\lambda} \in W_{0}^{1, \infty}\left(B_{R}, \mathbb{R}^{N}\right)$ of the above $v$ with $\lambda \in\left(k \Lambda, k^{q} \Lambda\right)$ for some integer $k>1$. In which case we can write 


$$
\begin{aligned}
f_{B_{R}} \mathbb{A}(\nabla \Pi(\theta) D u, D v)= & f_{B_{R}} \mathbb{A}\left(D u-A, \nabla \Pi(\theta)\left(D v-D v_{\lambda}\right)\right) \\
& +f_{B_{R}} \mathbb{A}\left(D u, \nabla \Pi(\theta) D v_{\lambda}\right) \\
& +f_{B_{R}} \mathbb{A}(\nabla \Pi(\theta) D u-D u, D v)=I+I I+I I I .
\end{aligned}
$$

Using Lemma 7.6 and $\alpha>1$ we bound the first term

$$
\begin{aligned}
I & \leqq L f_{B_{R}}|D u-A|\left|\nabla \Pi(\theta)\left(D v-D v_{\lambda}\right)\right| \mathbf{1}_{\left\{v \neq v_{\lambda}\right\}} \\
& \leqq c_{1} f_{B_{R}}|D u-A|^{p} \mathbf{1}_{\left\{v \neq v_{\lambda}\right\}}+\frac{1}{3 C_{0}} f_{B_{R}}|D v|^{q} \\
& \leqq c_{1}\left(f_{B_{R}}|D u-A|^{\alpha p}\right)^{\frac{1}{\alpha}}\left(\frac{\left|\left\{v \neq v_{\lambda}\right\}\right|}{\left|B_{R}\right|}\right)^{1-\frac{1}{\alpha}}+\frac{1}{3 C_{0}} f_{B_{R}}|D v|^{q}
\end{aligned}
$$

for some positive $c_{1}=c_{1}(n, N, p, \ell, L)$. Again, using the estimates from Lemma 7.6 we also bound

$$
\frac{\left|\left\{v_{\lambda} \neq v\right\}\right|}{\left|B_{R}\right|} \leqq c_{2} k^{-q}
$$

for some positive $c_{2}=c_{2}(n, N, p)$. Thus for every $0<\varepsilon<1$ we may choose $k=k(\varepsilon, \alpha, n, N, p, \ell, L)>0$ sufficiently large so that

$$
I \leqq \frac{\varepsilon}{2}\left(f_{B_{R}}|V(D u-A)|^{2 \alpha}\right)^{\frac{1}{\alpha}}+\frac{1}{3 C_{0}} f_{B_{R}}|D v|^{q} .
$$

As $u$ is a $\delta$-approximate tangential $\mathbb{A}$-harmonic map about $\theta$, Definition 7.2 implies, together with the bound $\left\|D v_{\lambda}\right\|_{\infty} \leqq \lambda \leqq k^{q} \Lambda$, Young's inequality and $p \geqq 2$, that the second term

$$
\begin{aligned}
I I & \leqq \delta\left(R^{\beta}+f_{B_{R}}|V(D u-A)|^{2}\right)^{\frac{1}{2}}\left\|D v_{\lambda}\right\|_{\infty} \\
& \leqq 2^{\frac{p-2}{2}} \delta k^{q}\left(R^{\beta}+\left(f_{B_{R}}|V(D u-A)|^{2}\right)^{\frac{p}{2}}+f_{B_{R}}|D v|^{q}\right) .
\end{aligned}
$$

Furthermore, we also have

$$
\begin{aligned}
I I I & \leqq L f_{B_{R}}|\nabla \Pi(\theta) D u-D u||D v| \\
& \leqq c_{3} f_{B_{R}}|V(\nabla \Pi(\theta) D u-D u)|^{2}+\frac{1}{3 C_{0}} f_{B_{R}}|D v|^{q}
\end{aligned}
$$

for some positive $c_{3}=c_{3}(n, N, p, \ell, L)$, where by Definition 7.2 we have

$$
f_{B_{R}}|V(\nabla \Pi(\theta) D u-D u)|^{2} \leqq \delta^{2} R+\delta^{2\left(1-\frac{1}{\alpha}\right)}\left(f_{B_{R}}|V(D u-A)|^{2 \alpha}\right)^{\frac{1}{\alpha}} .
$$


By choosing sufficiently small $\delta=O\left(\varepsilon^{2+\frac{1}{\alpha-1}}\right)$, that is a $\delta$ satisfying

$$
\delta \leqq K \varepsilon^{2+\frac{1}{\alpha-1}}
$$

for some $K=K(n, N, p, \alpha, \ell, L)>0$, we can bound $2^{\frac{p-2}{2}} \delta k^{q} \leqq \min \left\{\frac{\varepsilon}{2}, \frac{1}{3 C_{0}}\right\}$, $c_{3} \delta^{2\left(1-\frac{1}{\alpha}\right)} \leqq \frac{\varepsilon}{2}$ and, since $\varepsilon<1$, also $c_{3} \delta^{2} \leqq \frac{\varepsilon}{2}$ to conclude

$$
\begin{array}{rl}
f_{B_{R}} & \mathbb{A}(\nabla \Pi(\theta) D u, D v)-\frac{1}{C_{0}}|D v|^{q} \\
\leq \varepsilon\left[R^{\beta}+\left(f_{B_{R}}|V(D u-A)|^{2}\right)^{\frac{p}{2}}+\left(f_{B_{R}}|V(D u-A)|^{2 \alpha}\right)^{\frac{1}{\alpha}}\right] .
\end{array}
$$

If we denote $\Xi^{2}=f_{B_{R}}|D U|^{2}$, recalling $U \in W_{0}^{1,2}\left(B_{R}, \mathbb{R}^{N}\right)$ from the above, then by Lemma 7.6 there exists a Lipschitz approximation $U_{\mu} \in W_{0}^{1, \infty}\left(B_{R}, \mathbb{R}^{N}\right)$ to $U$ with $\mu \in\left(j \Xi, j^{2} \Xi\right)$ for some integer $j>1$. As the $\delta$-approximate tangential $\mathbb{A}$-harmonic condition about $\theta$ yields

$$
\begin{aligned}
f_{B_{R}} \mathbb{A}\left(D u, \nabla \Pi(\theta) D U_{\mu}\right) & \leqq \delta\left(R^{\beta}+f_{B_{R}}|V(D u-A)|^{2}\right)^{\frac{1}{2}}\left\|D U_{\mu}\right\|_{\infty} \\
& \leqq \delta j^{2}\left(R^{\beta}+f_{B_{R}}|V(D u-A)|^{2}+f_{B_{R}}|D U|^{2}\right)
\end{aligned}
$$

we can, like in the above estimates, choose an integer $j=j(\varepsilon, \alpha)>0$ sufficiently large and $\delta=O\left(\varepsilon^{2+\frac{1}{\alpha-1}}\right)$ sufficiently small such that

$$
\begin{aligned}
& \frac{1}{\ell} f_{B_{R}} \mathbb{A}(\nabla \Pi(\theta) D u, D U)-\frac{1}{2} f_{B_{R}}|D U|^{2} \\
& \quad \leqq \varepsilon\left[R^{\beta}+f_{B_{R}}|V(D u-A)|^{2}+\left(f_{B_{R}}|V(D u-A)|^{2 \alpha}\right)^{\frac{1}{\alpha}}\right] .
\end{aligned}
$$

The desired result now follows by combining the initial estimate (25) with both the $L^{2}$ and $L^{p}$-estimates and (27) for $\delta=O\left(\varepsilon^{2+\frac{1}{\alpha-1}}\right)$.

\section{Approximate Tangential Harmonicity}

In this section we establish the approximate harmonicity of holonomic $\mathfrak{F}$-minimisers $u \in W_{\text {loc }}^{1, p}(\Omega, \mathcal{M})$ as per Definition 7.2. To do so we first note that the strong quasiconvexity of (h2) implies

$$
\begin{aligned}
& \varepsilon \int_{B_{R}} D f^{0}(A)\left(\widehat{D \varphi)}+\varepsilon^{2} \int_{B_{R}} \int_{0}^{1}(1-t) D^{2} f^{0}(A+t \varepsilon D \varphi)(D \varphi, D \varphi) \mathrm{d} t\right. \\
& =\int_{B_{R}} f^{0}(A+\varepsilon D \varphi)-f^{0}(A) \geqq \gamma \varepsilon^{2} \int_{B_{R}}|D \varphi|^{2}
\end{aligned}
$$


for any matrix $A \in \mathbb{R}^{N n}$, any $\varphi \in C_{c}^{\infty}\left(B_{R}, \mathbb{R}^{N}\right)$ and fixed $\left(x_{0}, u_{0}\right) \in \Omega \times \mathbb{R}^{N}$, where $f^{0}(\xi)=f\left(x_{0}, u_{0}, \xi\right)$. By sending $\varepsilon \rightarrow 0$ the latter inequality becomes

$$
\int_{B_{R}} D^{2} f^{0}(A)(D \varphi, D \varphi) \geqq 2 \gamma \int_{B_{R}}|D \varphi|^{2} .
$$

So if, for any given $\Lambda>0$, we have $\left|(u)_{x_{0}, R}\right| \leqq \Lambda$ and $\left|(D u)_{x_{0}, R}\right| \leqq \Lambda$ on some $B_{R}\left(x_{0}\right) \subset \subset \Omega$, the bilinear form

$$
\mathbb{A}=D_{\xi \xi}^{2} f\left(x_{0},(u)_{x_{0}, R},(D u)_{x_{0}, R}\right)
$$

is then both bounded, with an upper bound $L=K_{\Lambda}$ from (4), and strongly elliptic in the sense of (23) with an ellipticity constant $\ell=2 \gamma$.

Given this one can use a linearisation strategy to prove the below approximate harmonicity result of Proposition 8.3 via Ekeland's variational principle and higher integrability. The linearisation strategy is applied first to the frozen problem yielding the following:

Lemma 8.1. Let $u \in W_{\mathrm{loc}}^{1, p}(\Omega, \mathcal{M}), p \geqq 2$, and suppose the integrand $f$ satisfies (h0), (h1), (h2). If $v \in\left(u+W_{0}^{1, p}\left(B_{R}\left(x_{0}\right), \mathbb{R}^{N}\right)\right) \cap W^{1, p}\left(B_{R}\left(x_{0}\right), \mathcal{M}\right)$, on a fixed $B_{R}\left(x_{0}\right) \subset \subset \Omega$ with $0<R<1$, satisfies

$$
\begin{aligned}
f_{B_{R}\left(x_{0}\right)} f\left(x_{0},(u)_{x_{0}, R}, D v\right) \leqq & f_{B_{R}\left(x_{0}\right)} f\left(x_{0},(u)_{x_{0}, R}, D w\right) \\
& +R^{\beta}\left(f_{B_{R}\left(x_{0}\right)}|D v-D w|^{p}\right)^{1 / p}
\end{aligned}
$$

for all $w \in\left(u+W_{0}^{1, p}\left(B_{R}\left(x_{0}\right), \mathbb{R}^{N}\right)\right) \cap W^{1, p}\left(B_{R}\left(x_{0}\right), \mathcal{M}\right)$ and some $0<\beta<1$, then for every $\Lambda>0$ there exists $C=C\left(n, N, p, \Lambda, K_{\Lambda}\right)>0$ such that

$$
\begin{aligned}
& \left|f_{B_{R}\left(x_{0}\right)} D_{\xi \xi}^{2} f\left(x_{0},(u)_{x_{0}, R},(D v)_{x_{0}, R}\right)\left(D v, \nabla \Pi\left(\Pi\left((v)_{x_{0}, R}\right)\right) D \phi\right)\right| \\
& \quad \leqq C\left(\mathcal{E}(R ; v)^{2}+\omega(\mathcal{E}(R ; v))^{\frac{1}{2}} \mathcal{E}(R ; v)+R^{\beta}\left(1+\mathcal{E}(R ; v)^{2}\right)^{\frac{1}{p}}\right)\|D \phi\|_{\infty}
\end{aligned}
$$

for all $\phi \in C_{c}^{\infty}\left(B_{R}\left(x_{0}\right), \mathbb{R}^{N}\right)$, whenever $\left|(u)_{x_{0}, R}\right| \leqq \Lambda,\left|(v)_{x_{0}, R}\right| \leqq \Lambda$, $\left|(D v)_{x_{0}, R}\right| \leqq \Lambda$ and $\Pi\left((v)_{x_{0}, R}\right)$ is well defined. ${ }^{8}$

Proof. Put $A=(D v)_{x_{0}, R}, \theta=\Pi\left((v)_{x_{0}, R}\right)$ and $f^{0}(\xi)=f\left(x_{0},(u)_{x_{0}, R}, \xi\right)$. Then write

$$
f_{B_{R}} D^{2} f^{0}(A)(D v, \nabla \Pi(\theta) D \phi)=I+I I+I I I
$$

${ }^{8}$ Here $\mathcal{E}(R ; v)=\left(f_{B_{R}}\left|V\left(D v-(D v)_{R}\right)\right|^{2}\right)^{\frac{1}{2}}$ denotes the excess with respect to $v$. 
into the parts

$I=f_{B_{R}} D f^{0}(D v) D(\nabla \Pi(v) \phi), \quad I I=f_{B_{R}} D f^{0}(D v)(\nabla \Pi(\theta) D \phi-D(\nabla \Pi(v) \phi))$ and

$$
\begin{aligned}
I I I & =f_{B_{R}} D^{2} f^{0}(A)(D v-A, \nabla \Pi(\theta) D \phi)+\left(D f^{0}(A)-D f^{0}(D v)\right)(\nabla \Pi(\theta) D \phi) \\
& =f_{B_{R}} \int_{0}^{1}\left[D^{2} f^{0}(A)-D^{2} f^{0}(A+t(D v-A))\right](D v-A, \nabla \Pi(\theta) D \phi) \mathrm{d} t \\
& =f_{B_{R}} \mathbf{1}_{\{|D v-A| \leqq 1\}} G+\mathbf{1}_{\{|D v-A|>1\}} G
\end{aligned}
$$

where

$$
G=\int_{0}^{1}\left[D^{2} f^{0}(A)-D^{2} f^{0}(A+t(D v-A))\right](D v-A, \nabla \Pi(\theta) D \phi) \mathrm{d} t .
$$

Now on $B_{R} \cap\{|D v-A|>1\}$ equations (2) and (4), via (31), imply

$$
|G| \leqq K_{\Lambda}|D v-A||D \phi|+c\left(1+|D v-A|^{p-1}\right)|D \phi| \leqq c|D v-A|^{p}|D \phi| .
$$

Moreover, on $B_{R} \cap\{|D v-A| \leqq 1\}$ Equation (32) and the modulus of continuity (5) imply $|G| \leqq c \omega(|D v-A|)|D v-A||D \phi|$. Hence by (11a) and (11f) we have

$$
\begin{aligned}
\left|f_{B_{R}} \mathbf{1}_{\{|D v-A| \leqq 1\}} G\right| & \leqq c\left(\frac{1}{\left|B_{R}\right|} \int_{B_{R} \cap\{|D v-A| \leqq 1\}} \omega(|D v-A|)^{2}\right)^{\frac{1}{2}} \mathcal{E}(R ; v)\|D \phi\|_{\infty} \\
& \leqq c\left[\omega\left(\frac{1}{\left|B_{R}\right|} \int_{B_{R} \cap\{|D v-A| \leqq 1\}}|D v-A|\right)\right]^{\frac{1}{2}} \mathcal{E}(R ; v)\|D \phi\|_{\infty} \\
& \leqq c \omega(\mathcal{E}(R ; v))^{\frac{1}{2}} \mathcal{E}(R ; v)\|D \phi\|_{\infty},
\end{aligned}
$$

where the second last inequality follows by the fact that $\omega$ is concave and at most 1 and the last inequality follows by Cauchy-Schwarz and the fact that $\omega$ is nondecreasing.

Expanding $I I$ yields

$$
I I=f_{B_{R}} D f^{0}(D v)\left(\nabla \Pi(\theta) D \phi-\nabla \Pi(v) D \phi-\nabla^{2} \Pi(v) D v \cdot \phi\right) .
$$

By the growth of (2), the bound $\|\phi\|_{\infty} \leqq c R\|D \phi\|_{\infty}$ and the Lipschitz properties of the retraction $\Pi$ (cf. Remark 2.3) we bound

$$
\begin{aligned}
|I I| & \leqq c\|D \phi\|_{\infty} f_{B_{R}}\left(1+|D v|^{p-1}\right)\left|v-(v)_{R}\right|+c R\|D \phi\|_{\infty} f_{B_{R}}|D v|+|D v|^{p} \\
& \leqq c R\|D \phi\|_{\infty} f_{B_{R}} 1+|D v|^{p} \\
& \leqq c R\|D \phi\|_{\infty}\left(1+\mathcal{E}(R ; v)^{2}\right) .
\end{aligned}
$$


Finally, by Corollary 6.4 and the bound $\|\phi\|_{\infty} \leqq c R\|D \phi\|_{\infty}$ we get

$$
\begin{aligned}
|I| \leqq R^{\beta}\left(f_{B_{R}}|D(\nabla \Pi(v) \phi)|^{p}\right)^{\frac{1}{p}} & \leqq c R^{\beta}\|D \phi\|_{\infty}\left(1+R^{p} f_{B_{R}}|D v|^{p}\right)^{\frac{1}{p}} \\
& \leqq c R^{\beta}\|D \phi\|_{\infty}\left(1+\mathcal{E}(R ; v)^{2}\right)^{\frac{1}{p}}
\end{aligned}
$$

Lemma 8.2. Let $u \in W^{1, p}\left(B_{R}, \mathcal{M}\right), p \geqq 2$, with $0<R<1$. Then for every $\Lambda>0$ and $\alpha>1$ there exists $c=c(n, N, p, \alpha, \Lambda)>0$ such that

$$
\begin{aligned}
f_{B_{R}}\left|V\left(\nabla \Pi\left(\Pi\left((u)_{R}\right)\right) D u-D u\right)\right|^{2} \leqq & c R^{2} f_{B_{R}} 1+|D u|^{p} \\
& +c\left(R^{2} f_{B_{R}} 1+|D u|^{p}\right)^{1-\frac{1}{\alpha}} \\
& \times\left(f_{B_{R}}\left|V\left(D u-(D u)_{R}\right)\right|^{2 \alpha}\right)^{\frac{1}{\alpha}},
\end{aligned}
$$

provided $\left|(D u)_{R}\right| \leqq \Lambda$ and $\Pi\left((u)_{R}\right)$ is well defined.

Proof. With $A=(D u)_{R}$ and $\theta=\Pi\left((u)_{R}\right)$ we note that

$\nabla \Pi(\theta) D u-\nabla \Pi(u) D u=(\nabla \Pi(\theta)-\nabla \Pi(u))(D u-A)+(\nabla \Pi(\theta)-\nabla \Pi(u)) A$, since $D u=D(\Pi(u))=\nabla \Pi(u) D u$. For any $\alpha>1$ we can, using (10), (11a), (11c) and the Lipschitz properties of the retraction $\Pi$ (cf. Remark 2.3), bound

$$
\begin{aligned}
& f_{B_{R}}|V(\nabla \Pi(\theta) D u-D u)|^{2} \\
& \quad \leqq c f_{B_{R}}\left(|u-\theta|^{2}|D u-A|^{2}+|u-\theta|^{p}|D u-A|^{p}\right)+c R^{2} f_{B_{R}}\left|V\left(\frac{u-\theta}{R}\right)\right|^{2} \\
& \leq c\left(f_{B_{R}}\|u\|_{\infty}^{\frac{2}{\alpha-1}}\left|u-(u)_{R}\right|^{2}+\|u\|_{\infty}^{\frac{p}{\alpha-1}}\left|u-(u)_{R}\right|^{p}\right)^{\frac{\alpha-1}{\alpha}} \\
& \quad \times\left(f_{B_{R}}|V(D u-A)|^{2 \alpha}\right)^{\frac{1}{\alpha}}+c R^{2} f_{B_{R}}|V(D u)|^{2} \\
& \leqq \\
& \quad c\left(R^{2} f_{B_{R}} 1+|D u|^{p}\right)^{1-\frac{1}{\alpha}}\left(f_{B_{R}}|V(D u-A)|^{2 \alpha}\right)^{\frac{1}{\alpha}}+c R^{2} f_{B_{R}} 1+|D u|^{p},
\end{aligned}
$$

since $u \in L^{\infty}$ (as $\mathcal{M}$ is compact).

Now for $v-u \in W_{0}^{1, p}\left(B_{R}\left(x_{0}\right), \mathbb{R}^{N}\right)$ we have $(D u)_{x_{0}, R}=(D v)_{x_{0}, R}$ by the divergence theorem. So by (11a) and (11e) we find

$$
\mathcal{E}(R ; v)^{2} \leqq \mathcal{E}(R ; u)^{2}+c f_{B_{R}\left(x_{0}\right)}|D u-D v|^{2}+|D u-D v|^{p},
$$


whenever $\left|(D u)_{x_{0}, R}\right| \leqq \Lambda$. Given this we can, using the fact that the minimiser $v$ of the frozen problem is close to the holonomic $\mathfrak{F}$-minimiser $u$ in the sense of (21) via Ekeland's variational principle, establish the following approximate tangential harmonicity result.

Proposition 8.3. If for every $\Lambda>0$, under the assumptions of Theorem 1.2, the map $u \in W_{\mathrm{loc}}^{1, p}(\Omega, \mathcal{M})$ is an holonomic $\mathfrak{F}$-minimiser with

$$
\left|(u)_{x_{0}, 2 R}\right| \leqq \Lambda, \quad\left|(D u)_{x_{0}, 2 R}\right| \leqq \Lambda, \quad\left(f_{B_{2 R}\left(x_{0}\right)}|D u|^{p}\right)^{\frac{1}{p}} \leqq \Lambda+1
$$

on some $B_{2 R}\left(x_{0}\right) \subset \subset \Omega$ with $R=R(n, N, p, \Lambda)$ sufficiently small, then for any $\alpha>1$ there exists $C=C\left(n, N, p, \Lambda, K_{\Lambda}, \alpha\right)>0$ such that

$$
\begin{aligned}
& f_{B_{R}\left(x_{0}\right)}\left|V\left(D u-\nabla \Pi\left(\Pi\left((u)_{x_{0}, R}\right)\right) D u\right)\right|^{2} \\
& \quad \leqq C e^{2} R+C e^{2\left(1-\frac{1}{\alpha}\right)}\left(f_{B_{R}\left(x_{0}\right)}\left|V\left(D u-(D u)_{x_{0}, R}\right)\right|^{2 \alpha}\right)^{\frac{1}{\alpha}}
\end{aligned}
$$

and

$$
\begin{aligned}
& \left|f_{B_{R}\left(x_{0}\right)} D_{\xi \xi}^{2} f\left(x_{0},(u)_{x_{0}, R},(D u)_{x_{0}, R}\right)\left(D u, \nabla \Pi\left(\Pi\left((u)_{x_{0}, R}\right)\right) D \phi\right)\right| \\
& \quad \leqq C e\left(e+\omega(e)^{\frac{1}{2}}\right)\|D \phi\|_{\infty}
\end{aligned}
$$

for all $\phi \in C_{c}^{\infty}\left(B_{R}\left(x_{0}\right), \mathbb{R}^{N}\right)$, where $e=\left(R^{\beta}+\mathcal{E}(R ; u)^{2}\right)^{\frac{1}{2}}$ for some $0<\beta<1$.

Proof. Using the initial assumptions, the result of Section 6 yields a minimiser $v \in\left(u+W_{0}^{1, p}\left(B_{R}\left(x_{0}\right), \mathbb{R}^{N}\right)\right) \cap W^{1, p}\left(B_{R}\left(x_{0}\right), \mathcal{M}\right)$ of (22) which also satisfies (21). Furthermore, from (21) and the initial assumptions, the Poincaré inequality implies

$$
\begin{aligned}
& \operatorname{dist}\left((u)_{R}, \mathcal{M}\right) \leqq\left(f_{B_{R}}\left|u-(u)_{R}\right|^{p}\right)^{\frac{1}{p}} \leqq c R(1+\Lambda) \\
& \operatorname{dist}\left((v)_{R}, \mathcal{M}\right) \leqq\left(f_{B_{R}}\left|v-(v)_{R}\right|^{p}\right)^{\frac{1}{p}} \leqq c R\left(1+\Lambda^{p}\right)^{\frac{1}{p}},
\end{aligned}
$$

in which case the expressions $\Pi\left((u)_{R}\right)$ and $\Pi\left((v)_{R}\right)$ are well defined for $0<R<$ $c^{-1}\left(1+\Lambda^{p}\right)^{-\frac{1}{p}} \rho$, where $\rho=\operatorname{reach}(\mathcal{M})>0$ denote the positive reach of $\mathcal{M}$ (cf. Section 2.2). Similarly, we have $\left|(v)_{R}\right| \leqq c(1+\Lambda),\left|(D v)_{R}\right| \leqq c(1+\Lambda)$ by (21), the Poincaré inequality and the initial assumptions. With $\theta_{u}=\Pi\left((u)_{R}\right)$, $\theta_{v}=\Pi\left((v)_{R}\right)$ and $f^{0}(\xi)=f\left(x_{0},(u)_{x_{0}, R}, \xi\right)$ we observe, using $(D u)_{R}=(D v)_{R}$, that

$$
f_{B_{R}} D^{2} f^{0}\left((D u)_{R}\right)\left(D u, \nabla \Pi\left(\theta_{u}\right) D \phi\right)
$$




$$
\begin{aligned}
= & f_{B_{R}} D^{2} f^{0}\left((D v)_{R}\right)\left(D v, \nabla \Pi\left(\theta_{v}\right) D \phi\right) \\
& +f_{B_{R}} D^{2} f^{0}\left((D u)_{R}\right)\left(D v,\left(\nabla \Pi\left(\theta_{u}\right)-\nabla \Pi\left(\theta_{v}\right)\right) D \phi\right) \\
& +f_{B_{R}} D^{2} f^{0}\left((D u)_{R}\right)\left(D u-D v, \nabla \Pi\left(\theta_{u}\right) D \phi\right) .
\end{aligned}
$$

Then using (4), (5) and the Lipschitz properties of the retraction $\Pi$ (cf. Remark 2.3) we bound

$$
\begin{aligned}
& \left|f_{B_{R}} D^{2} f^{0}\left((D u)_{R}\right)\left(D u, \nabla \Pi\left(\theta_{u}\right) D \phi\right)\right| \\
& \leq\left|f_{B_{R}} D^{2} f^{0}\left((D v)_{R}\right)\left(D v, \nabla \Pi\left(\theta_{v}\right) D \phi\right)\right| \\
& \quad+C\left(f_{B_{R}}|D v|\right)\left(f_{B_{R}}|u-v|\right)\|D \phi\|_{\infty} \\
& \quad+C\left(f_{B_{R}}|D u-D v|^{p}\right)^{\frac{1}{p}}\|D \phi\|_{\infty} .
\end{aligned}
$$

The result now follows from Lemmas 8.1, 8.2 and the Poincaré inequality, since $\mathcal{E}(R ; v)^{2} \leqq \mathcal{E}(R ; u)^{2}+C R^{\beta}$ by (33) together with (21).

\section{Reverse Hölder-Type Inequalities}

We establish reverse Hölder-type inequalities following a Caccioppoli inequality of the second kind argument using strict quasiconvexity and minimality via the comparison map construction from Section 4.

Lemma 9.1. Let $\mathcal{M}$ be a connected compact manifold without boundary and $\pi: \widetilde{\mathcal{M}} \rightarrow \mathcal{M}$ be its universal cover such that $\widetilde{\mathcal{M}}$ is compact and $\ell$-connected for some integer $\ell \geqq 1$. Suppose the integrand $f$ satisfies (h0), (h1), (h2), (h3) for $2 \leqq p<\ell+2$ and $u \in W_{\mathrm{loc}}^{1, p}(\Omega, \mathcal{M})$ is an holonomic $\mathfrak{F}$-minimiser. Then for every $\Lambda>0$ there exists $C=C\left(n, N, p, \gamma, \Gamma, \Lambda, K_{\Lambda+1}\right)>0$ and $R_{1}=R_{1}(n, N, p, \Lambda)$ such that

$$
\begin{aligned}
f_{B_{R / 2}\left(x_{0}\right)}|V(D u-A)|^{2} \leqq & C\left(f_{B_{R}\left(x_{0}\right)}|V(D u-A)|^{2 *}\right)^{2 / 2_{*}} \\
& +C R^{2} f_{B_{R}\left(x_{0}\right)}\left(1+|D u|^{p}\right) \\
& +C f_{B_{R}\left(x_{0}\right)}|V(\nabla \Pi(u) A-A)|^{2} \\
& +C f_{B_{R}\left(x_{0}\right)} \omega_{\mu}\left(R+\left|u-u_{0}\right|+\Phi_{1}(D u)\right)\left(1+|D u|^{p}\right)
\end{aligned}
$$


for any $B_{R}\left(x_{0}\right) \subset \subset \Omega$ with $0<R<R_{1}$, any $u_{0} \in \mathbb{R}^{N}$ with $\left|u_{0}\right| \leqq \Lambda+1$ and any $A \in \mathbb{R}^{N n}$ with $|A| \leqq \Lambda$.

Remark 9.2. Here we define the operator $\Phi_{\alpha}$ on $L^{1}\left(B_{R}\left(x_{0}\right)\right)$ for $0<\alpha<n$ by the Riesz potential

$$
\Phi_{\alpha} f(x)=\frac{1}{c_{n}(\alpha)} \int_{B_{R}\left(x_{0}\right)}|x-y|^{\alpha-n}|f(y)| \mathrm{d} y,
$$

where $c_{n}(\alpha)=2^{\alpha} \pi^{\frac{n}{2}} \Gamma\left(\frac{\alpha}{2}\right) / \Gamma\left(\frac{n-\alpha}{2}\right)$. We note that $\Phi_{\alpha}$ is well defined and maps $L^{1}\left(B_{R}\left(x_{0}\right)\right)$ into itself and has the following semi-group property

$$
\Phi_{\alpha}\left(\Phi_{\beta}(f)\right)=\Phi_{\alpha+\beta}(f)
$$

for $\alpha, \beta>0$ with $\alpha+\beta<n$ (cf. [77, §7]).

Proof. Let $\frac{R}{2} \leqq r<s \leqq R$ and choose a cut-off function $\eta \in C_{c}^{\infty}(\Omega)$ such that $\eta=1$ on $B_{r}\left(x_{0}\right), \eta=0$ on $\Omega \backslash B_{s}\left(x_{0}\right), 0 \leqq \eta \leqq 1$ and $|D \eta| \leqq C /(s-r)$. Define $\phi_{1}=\eta \bar{u}$ and $\phi_{2}=(1-\eta) \bar{u}$, where $\bar{u}(x)=u(x)-(u)_{x_{0}, R}-A \cdot\left(x-x_{0}\right)$, so that $D \phi_{1}+D \phi_{2}=D u-A$. By setting $f^{0}(\xi)=f\left(x_{0}, u_{0}, \xi\right)$ and using the quasiconvexity of (h2) we obtain

$$
\begin{aligned}
\gamma \int_{B_{s}}\left(1+|A|^{2}+\left|D \phi_{1}\right|^{2}\right)^{\frac{p-2}{2}}\left|D \phi_{1}\right|^{2} \leqq & \int_{B_{s}} f^{0}\left(D u-D \phi_{2}\right)-f^{0}(D u) \\
& +\int_{B_{s}} f^{0}(D u)-f^{0}\left(A+D \phi_{2}\right) \\
& +\int_{B_{s}} f^{0}\left(A+D \phi_{2}\right)-f^{0}(A) \\
& =I+I I+I I I .
\end{aligned}
$$

By introducing the auxiliary functional $\mathfrak{F}_{s, A}(w)=\int_{B_{s}} \mathrm{~F}(D w) \mathrm{d} x$, with integrand $\mathrm{F}(\xi)=f^{0}(\xi+A)-f^{0}(A)-D f^{0}(A)(\xi)$, we note that

$$
\begin{aligned}
I I= & \int_{B_{s}} f^{0}(D u)-f^{0}(A)-D f^{0}(A)\left(D \phi_{1}+D \phi_{2}\right) \\
& +\int_{B_{s}} \int_{0}^{1}\left(D f^{0}(A)-D f^{0}\left(A+\lambda D \phi_{2}\right)\right) \cdot D \phi_{2} \mathrm{~d} \lambda=\mathfrak{F}_{s, A}(\bar{u})+I I^{\prime}
\end{aligned}
$$

since $\phi_{1}=0$ on $\partial B_{s}$. Now as

$$
I+I I I=\int_{B_{s}} \int_{0}^{1}\left(D f^{0}\left(A+\lambda D \phi_{2}\right)-D f^{0}\left(D u-\lambda D \phi_{2}\right)\right) \cdot D \phi_{2} \mathrm{~d} \lambda,
$$

it follows that $I+I I+I I I=\mathfrak{F}_{s, A}(\bar{u})+I I I^{\prime}$, where

$$
I I I^{\prime}=\int_{B_{s}} \int_{0}^{1}\left(D f^{0}(A)-D f^{0}\left(D u-\lambda D \phi_{2}\right)\right) \cdot D \phi_{2} \mathrm{~d} \lambda .
$$


Looking to the auxiliary functional we have, by the minimality of $u$,

$$
\begin{aligned}
\mathfrak{F}_{s, A}(\bar{u})= & \int_{B_{s}} f^{0}(D u)-f^{0}(A)-D f^{0}(A)(D u-A) \\
\leqq & \int_{B_{s}} f^{0}(D v)-f^{0}(A)-D f^{0}(A)(D v-A) \\
& +\int_{B_{s}} f^{0}(D u)-f(x, u, D u)+\int_{B_{s}} f(x, v, D v)-f^{0}(D v)
\end{aligned}
$$

for any $v \in\left(u+W_{0}^{1, p}\left(B_{s}, \mathbb{R}^{N}\right)\right) \cap W^{1, p}\left(B_{s}, \mathcal{M}\right)$. Furthermore, using (11a) one easily bounds $\mathrm{F}(\xi) \leqq C|V(\xi)|^{2}$ (since for $|\xi|>1, \mathrm{~F}(\xi) \leqq C|\xi|^{p}$ by (2) and for $|\xi| \leqq 1$ write $\mathrm{F}(\xi)=\int_{0}^{1}(1-\lambda) D^{2} f^{0}(A+\lambda \xi)(\xi, \xi) \mathrm{d} \lambda$ so that $|\mathrm{F}(\xi)| \leqq K_{\Lambda+1}|\xi|^{2}$ by (4) since $\left|u_{0}\right| \leqq \Lambda+1$ also). It then follows by (h3) that

$$
\begin{aligned}
\mathfrak{F}_{s, A}(\bar{u}) \leqq & C \int_{B_{s}}|V(D v-A)|^{2}+C \int_{B_{s}} \omega_{\mu}\left(\left|x-x_{0}\right|+\left|u-u_{0}\right|\right)\left(1+|D u|^{p}\right) \\
& +C \int_{B_{s}} \omega_{\mu}\left(\left|x-x_{0}\right|+\left|v-u_{0}\right|\right)\left(1+|D v|^{p}\right) .
\end{aligned}
$$

To estimate $I I I^{\prime}$ we note that the integrand on $B_{s} \cap\left\{|D u-A|+\left|D \phi_{2}\right|>1\right\}$ can be bounded, using (2) and the fact that $|A| \leqq \Lambda$, by

$$
\left|D f^{0}(A)-D f^{0}\left(D u-\lambda D \phi_{2}\right)\right|\left|D \phi_{2}\right| \leqq C\left(|D u-A|+\left|D \phi_{2}\right|\right)^{p-1}\left|D \phi_{2}\right| .
$$

On $B_{s} \cap\left\{|D u-A|+\left|D \phi_{2}\right| \leqq 1\right\}$ we use (4), noting that $\left|D u-\lambda D \phi_{2}\right| \leqq \Lambda+1$ and $\left|u_{0}\right| \leqq \Lambda+1$, to obtain

$$
\left|D f^{0}(A)-D f^{0}\left(D u-\lambda D \phi_{2}\right)\right|\left|D \phi_{2}\right| \leqq K_{\Lambda+1}\left(|D u-A|+\left|D \phi_{2}\right|\right)\left|D \phi_{2}\right| .
$$

Now as $D \phi_{2}=(1-\eta)(D u-A)-\bar{u} \otimes D \eta$ we have $\left|V\left(D \phi_{2}\right)\right| \leqq C(\mid V(D u-$ $\left.A)|+| V\left(\frac{\bar{u}}{s-r}\right) \mid\right)$ by (11c). This together with the fact that $D \phi_{2}=0$ on $B_{r}$ implies

$$
I I I^{\prime} \leqq C \int_{B_{s} \backslash B_{r}}|V(D u-A)|^{2}+\left|V\left(\frac{\bar{u}}{s-r}\right)\right|^{2} .
$$

Furthermore, we bound the left-hand side of (34) on $B_{r}$ from below, using (11d) and (11f), by

$$
\begin{aligned}
\gamma\left(1+|A|^{2}+\left|D \phi_{1}\right|^{2}\right)^{\frac{p-2}{2}}\left|D \phi_{1}\right|^{2} & \geqq C\left(1+|A|^{2}+|D u|^{2}\right)^{\frac{p-2}{2}}|D u-A|^{2} \\
& \geqq C|V(D u)-V(A)|^{2} \\
& \geqq C|V(D u-A)|^{2},
\end{aligned}
$$

where $C=C(n, N, p, \gamma, \Lambda)$. In which case, by (h3) we conclude

$$
\begin{aligned}
\int_{B_{r}}|V(D u-A)|^{2} \leqq & C \int_{B_{s} \backslash B_{r}}|V(D u-A)|^{2}+C \int_{B_{s}}|V(D v-A)|^{2}+\left|V\left(\frac{\bar{u}}{s-r}\right)\right|^{2} \\
& +C \int_{B_{s}} \omega_{\mu}\left(\left|x-x_{0}\right|+\left|u-u_{0}\right|\right)\left(1+|D u|^{p}\right) \\
& +C \int_{B_{s}} \omega_{\mu}\left(\left|x-x_{0}\right|+\left|v-u_{0}\right|\right)\left(1+|D v|^{p}\right)
\end{aligned}
$$


for any $v \in\left(u+W_{0}^{1, p}\left(B_{s}, \mathbb{R}^{N}\right)\right) \cap W^{1, p}\left(B_{s}, \mathcal{M}\right)$.

Now if we let $a: \mathbb{R}^{n} \rightarrow \mathbb{R}^{N}$ denote the linear map

$$
x \mapsto A \cdot\left(x-x_{0}\right)
$$

on $B_{R}$, then $\operatorname{dist}(u-a, \mathcal{M}) \leqq\|a\|_{\infty} \leqq \Lambda R$ since $|A| \leqq \Lambda$. In which case, using the retraction $\Pi$ from a tubular neighbourhood onto $\mathcal{M}$, the expression

$$
\widehat{u}=\Pi(u-a)
$$

is well defined for $0<R<\Lambda^{-1} \rho$, where $\rho>0$ denote the positive reach of $\mathcal{M}$. From the lifting of Theorem 4.1 we have $\widehat{u}=\pi \circ \widehat{\varphi}$ for some $\widehat{\varphi} \in W^{1, p}\left(B_{s}, \widetilde{\mathcal{M}}\right)$. Then by Lemma 4.9, (10) and (11b) there exists $\widehat{v} \in\left(\widehat{u}+W_{0}^{1, p}\left(B_{s}, \mathbb{R}^{N}\right)\right) \cap$ $W^{1, p}\left(B_{s}, \mathcal{M}\right)$ such that

$$
\int_{B_{S}}|V(D \widehat{v})|^{2} \leqq C \int_{B_{S} \backslash B_{r}}|V(D \widehat{u})|^{2}+C \frac{R^{n+p}}{(s-r)^{p}}\left(f_{B_{R}}|V(D \widehat{u})|^{2_{*}}\right)^{2 / 2_{*}}
$$

with

$$
|\widehat{v}(x)-\widehat{u}(x)| \leqq C \int_{B_{s}}|x-y|^{1-n}\left(|D \widehat{u}(y)|+\frac{\left|\widehat{\varphi}(y)-(\widehat{\varphi})_{B_{s}}\right|}{s-r}\right) \mathrm{d} y
$$

for almost everywhere $x \in B_{s}$.

As $\operatorname{dist}(u-\widehat{u}+\widehat{v}, \mathcal{M}) \leqq|u-\widehat{u}| \leqq \operatorname{Lip} \Pi\|a\|_{\infty}$ by Lipschitz properties of the retraction $\Pi$, the competitor $v \in\left(u+W_{0}^{1, p}\left(B_{S}, \mathbb{R}^{N}\right)\right) \cap W^{1, p}\left(B_{S}, \mathcal{M}\right)$ to the minimiser $u$ can be defined by setting

$$
v=\Pi(u-\widehat{u}+\widehat{v})
$$

for all $0<R<(\operatorname{Lip} \Pi)^{-1} \Lambda^{-1} \rho=R_{1}$. In particular, as $\widehat{v}=\widehat{u}$ on $\partial B_{S}$ by construction we have $v-u \in W_{0}^{1, p}\left(B_{s}, \mathbb{R}^{N}\right)$. By writing

$$
\begin{aligned}
D v-A= & \nabla \Pi(u-\widehat{u}+\widehat{v})[(\nabla \Pi(u)-\nabla \Pi(u-a)) D u \\
& +(\nabla \Pi(u-a)-\nabla \Pi(u)) A+\nabla \Pi(u) A-A] \\
& +[\nabla \Pi(u-\widehat{u}+\widehat{v})-\nabla \Pi(u)] A+\nabla \Pi(u) A-A+\nabla \Pi(u-\widehat{u}+\widehat{v}) D \widehat{v}
\end{aligned}
$$

we can bound

$$
|D v-A| \leqq c\|a\|_{\infty}(\Lambda+|D u|)+c \Lambda|\widehat{u}-\widehat{v}|+c|\nabla \Pi(u) A-A|+c|D \widehat{v}|
$$

by Remark 2.3. So by the Poincaré inequality, (11b) and (11c) we have

$$
\int_{B_{s}}|V(D v-A)|^{2} \leqq C \int_{B_{s}}\left(|V(D \widehat{v})|^{2}+|V(\nabla \Pi(u) A-A)|^{2}+R^{2}\left(1+|D u|^{p}\right)\right)
$$


for some positive $C=C(n, N, p, \Lambda)$. To estimate the final term in (35), we first note that $\left|v-u_{0}\right| \leqq\left|u-u_{0}\right|+|v-u| \leqq\left|u-u_{0}\right|+c|\widehat{u}-\widehat{v}|$ by the Lipschitz properties of $\Pi$. Then by (36) and [47, Lem. 7.16] we have

$$
\begin{aligned}
|\widehat{v}(x)-\widehat{u}(x)| \leqq & C \int_{B_{s}}|x-y|^{1-n}|D \widehat{u}(y)| \mathrm{d} y \\
& +\frac{C}{s-r} \int_{B_{s}}|x-y|^{1-n}\left(\int_{B_{s}}|y-z|^{1-n}|D \widehat{u}(z)| d z\right) \mathrm{d} y \\
\leqq & C\left(R+\Phi_{1}(D u)\right)+\frac{C}{s-r}\left(R^{2}+\Phi_{1}\left(\Phi_{1}(D u)\right)\right)
\end{aligned}
$$

for almost everywhere $x \in B_{s}$, since $|D \widehat{\varphi}|=|D \widehat{u}| \leqq c|D u-A|$. Moreover, by Remark 9.2 we note, for $n>2$, that $\Phi_{1} \circ \Phi_{1}=\Phi_{2}$ and $\Phi_{2}(D u) \leqq$ $C \operatorname{diam}\left(B_{R}\right) \Phi_{1}(D u)$ almost everywhere by the definition, whereas for $n=2$ we have $\Phi_{1 / 2} \circ \Phi_{1}=\Phi_{3 / 2}$ so that $\Phi_{1} \circ \Phi_{1}(D u) \leqq C \operatorname{diam}\left(B_{R}\right)^{\frac{1}{2}} \Phi_{1 / 2} \circ \Phi_{1}(D u)$ and $\Phi_{3 / 2}(D u) \leqq C \operatorname{diam}\left(B_{R}\right)^{\frac{1}{2}} \Phi_{1}(D u)$ almost everywhere. Thus we obtain the pointwise bound

$$
|\widehat{v}-\widehat{u}| \leqq C \frac{R}{s-r}\left(R+\Phi_{1}(D u)\right) .
$$

In which case we find, as $\omega_{\mu}(t)=\min \left(1, t^{\mu}\right)$ with $0<\mu \leqq 1$ and $p \geqq 2$, that

$$
\begin{aligned}
& \int_{B_{s}} \omega_{\mu}\left(\left|x-x_{0}\right|+\left|v-u_{0}\right|\right)\left(1+|D v|^{p}\right) \\
& \quad \leqq C \int_{B_{s}}|V(D \widehat{v})|^{2}+C \int_{B_{s}} \omega_{\mu}\left(\left|x-x_{0}\right|+\left|v-u_{0}\right|\right)\left(1+|D u|^{p}\right),
\end{aligned}
$$

since $|D v-A| \leqq c(1+|D u|)+c|D \widehat{v}|$ by (37) together with the fact that $\mathcal{M}$ is compact. Furthermore, from (38) we bound

$$
\begin{aligned}
& \int_{B_{S}} \omega_{\mu}\left(\left|x-x_{0}\right|+\left|v-u_{0}\right|\right)\left(1+|D u|^{p}\right) \\
& \quad \leqq C\left(\frac{R}{s-r}\right)^{\mu} \int_{B_{s}} \omega_{\mu}\left(R+\left|u-u_{0}\right|+\Phi_{1}(D u)\right)\left(1+|D u|^{p}\right) .
\end{aligned}
$$

Finally, by combining these estimates together with (35), and using (10), we obtain

$$
\begin{aligned}
\int_{B_{r}}|V(D u-A)|^{2} \leqq & C \int_{B_{s} \backslash B_{r}}|V(D u-A)|^{2}+C \frac{R^{n+p}}{(s-r)^{p}}\left(f_{B_{R}}|V(D u-A)|^{2 *}\right)^{2 / 2_{*}} \\
& +C\left(\frac{R}{s-r}\right)^{\mu} \int_{B_{s}} \omega_{\mu}\left(R+\left|u-u_{0}\right|+\Phi_{1}(D u)\right)\left(1+|D u|^{p}\right) \\
& +C \int_{B_{R}}|V(\nabla \Pi(u) A-A)|^{2}+R^{2}\left(1+|D u|^{p}\right)
\end{aligned}
$$

for some positive $C=C\left(n, N, p, \gamma, \Gamma, \Lambda, K_{\Lambda+1}\right)$. The result now follows by Widman's hole filling trick and an application of the standard iteration lemma (cf. [40, Lem. 1.1]). 
Given the above result one can apply Gehring's lemma to obtain the following:

Corollary 9.3. Let $\mathcal{M}$ be a connected compact manifold without boundary and $\pi: \widetilde{\mathcal{M}} \rightarrow \mathcal{M}$ be its universal cover such that $\widetilde{\mathcal{M}}$ is compact and $\ell$-connected for some integer $\ell \geqq 1$. Suppose the integrand $f$ satisfy $(h 0)$, (h1), (h2) and (h3) for $2 \leqq p<2+\ell$. If for every $\Lambda>0$ the map $u \in W_{\mathrm{loc}}^{1, p}(\Omega, \mathcal{M})$ is an holonomic $\mathfrak{F}$-minimiser with

$$
\left|(u)_{x_{0}, 2 R}\right| \leqq \Lambda, \quad\left|(D u)_{x_{0}, 2 R}\right| \leqq \Lambda, \quad\left(f_{B_{2 R}\left(x_{0}\right)}|D u|^{p}\right)^{\frac{1}{p}} \leqq \Lambda+1
$$

on some $B_{2 R}\left(x_{0}\right) \subset \subset \Omega$ for $R=R(n, N, p, \Lambda)$ sufficiently small, then there exists $q=q\left(n, N, p, \gamma, \Gamma, \Lambda, K_{\Lambda+1}\right)>p$ and $C=C\left(n, N, p, \gamma, \Gamma, \Lambda, K_{\Lambda+1}\right)$ such that

$$
\begin{aligned}
\left(f_{B_{\frac{R}{2}}\left(x_{0}\right)} \mid V\left(D u-\left.(D u)_{\left.x_{0}, \frac{R}{2}\right)}\right|^{\frac{4 q}{p+q}}\right)^{\frac{p+q}{2 q}}\right. & \leqq \\
& C R^{\frac{\mu}{2}\left(1-\frac{p}{q}\right)} \\
& +C f_{B_{R}\left(x_{0}\right)}\left|V\left(D u-(D u)_{x_{0}, R}\right)\right|^{2} .
\end{aligned}
$$

Proof. For every $\Xi>0$ there exists $C>0$ and $0<R_{1} \ll 1$ such that the inequality of Lemma 9.1 holds on all balls $B_{R}\left(x_{0}\right) \subset \subset \Omega$ with $0<R<R_{1}$ and any $u_{0} \in \mathbb{R}^{N}$, $A \in \mathbb{R}^{N n}$ such that $\left|u_{0}\right| \leqq \Xi+1,|A| \leqq \Xi$. Then by applying Gehring's lemma (cf. [45, Prop. 5.1]), there exists $\alpha_{0}=\alpha_{0}\left(n, N, p, \gamma, \Gamma, \Xi, K_{\Xi+1}\right)>1$ such that for any $1<\alpha \leqq \alpha_{0}$, any $u_{0} \in \mathbb{R}^{N}$ with $\left|u_{0}\right| \leqq \Xi+1$ and any $A \in \mathbb{R}^{N n}$ with $|A| \leqq \Xi$, we have

$$
\begin{aligned}
\left(f_{B_{R / 2}}|V(D u-A)|^{2 \alpha}\right)^{\frac{1}{2 \alpha}} \leqq & C\left(f_{B_{R}}|V(D u-A)|^{2}\right)^{\frac{1}{2}} \\
& +C R\left(f_{B_{R}} 1+|D u|^{\alpha p}\right)^{\frac{1}{2 \alpha}} \\
& +C\left(f _ { B _ { R } } \omega _ { \mu } \left(R+\left|u-u_{0}\right|\right.\right. \\
& \left.\left.+\Phi_{1}(D u)\right)^{\alpha}\left(1+|D u|^{\alpha p}\right)\right)^{\frac{1}{2 \alpha}} \\
& +C\left(f_{B_{R}}|V(\nabla \Pi(u) A-A)|^{2 \alpha}\right)^{\frac{1}{2 \alpha}}
\end{aligned}
$$

on a ball $B_{R}=B_{R}\left(x_{0}\right) \subset \subset \Omega$ with $0<R \ll 1$ sufficiently small. Note that the integrands of Lemma 9.1 on the right-hand side which are dependent on $R$ can be handled by 'freezing $R$ ' for a moment (as they are monotonically increasing in $R$ ) before applying Gehring's lemma on increasing supports (cf. [46, p. 200]). 
Now from the local higher integrability of Lemma 5.1 there exists $q>p$ such that $D u \in L_{\text {loc }}^{q}$ with

$$
f_{B_{R}}|D u|^{q} \leqq c\left(f_{B_{2 R}} 1+|D u|^{p}\right)^{\frac{q}{p}} .
$$

Thus we can set $\alpha_{0}=q / p$ without loss of generality. Also by setting $u_{0}=(u)_{x_{0}, R}$ and $A=(D u)_{x_{0}, R / 2}$ the initial assumptions imply $|A| \leqq 4^{n} f_{B_{2 R}}|D u| \leqq 4^{n}(1+\Lambda)$ and $\left|u_{0}\right| \leqq\left|(u)_{2 R}\right|+f_{B_{R}}\left|u-(u)_{2 R}\right| \leqq c(1+\Lambda)$ by the Poincaré inequality. Hence it suffices to take $\Xi=c(1+\Lambda)$ for some $c>4^{n}$. Then for any $1<\alpha<\alpha_{0}$ we estimate

$$
\begin{aligned}
& f_{B_{R}} \omega_{\mu}\left(R+\left|u-u_{0}\right|+\Phi_{1}(D u)\right)^{\alpha}\left(1+|D u|^{\alpha p}\right) \\
& \quad \leqq R^{-\mu \frac{\alpha p}{q}} \omega_{\mu}\left(f_{B_{R}} R+\left|u-u_{0}\right|+\Phi_{1}(D u)\right)+R^{\mu\left(1-\frac{\alpha p}{q}\right)} f_{B_{R}} 1+|D u|^{q},
\end{aligned}
$$

since $\omega_{\mu}$ is concave and no greater than 1 . Furthermore we find the Poincaré inequality, standard potential estimates (cf. [47, Lem. 7.12]) and higher integrability imply

$$
f_{B_{R}} R+\left|u-u_{0}\right|+\Phi_{1}(D u) \leqq c R\left(f_{B_{R}} 1+|D u|^{p}\right)^{\frac{1}{p}} .
$$

Then from (40) we bound

$$
\begin{aligned}
& f_{B_{R}} \omega_{\mu}\left(R+\left|u-u_{0}\right|+\Phi_{1}(D u)\right)^{\alpha}\left(1+|D u|^{\alpha p}\right) \\
& \quad \leqq c R^{\mu\left(1-\frac{\alpha p}{q}\right)} \omega_{\mu}\left(\left(f_{B_{2 R}} 1+|D u|^{p}\right)^{\frac{1}{p}}\right)+c R^{\mu\left(1-\frac{\alpha p}{q}\right)}\left(f_{B_{2 R}} 1+|D u|^{p}\right)^{\frac{q}{p}} .
\end{aligned}
$$

In particular, if we set $\alpha=2 q /(p+q)$ the relation $1<\alpha<q / p$ is satisfied with

$$
\begin{aligned}
& \left(f_{B_{R}} \omega_{\mu}\left(R+\left|u-u_{0}\right|+\Phi_{1}(D u)\right)^{\alpha}\left(1+|D u|^{\alpha p}\right)\right)^{\frac{1}{2 \alpha}} \\
& \leqq c R^{\frac{\mu}{4}\left(1-\frac{p}{q}\right)} \omega_{\mu}\left(\left(f_{B_{2 R}} 1+|D u|^{p}\right)^{\frac{1}{p}}\right)^{\frac{p+q}{4 q}} \\
& \quad+c R^{\frac{\mu}{4}\left(1-\frac{p}{q}\right)}\left(f_{B_{2 R}} 1+|D u|^{p}\right)^{\frac{p+q}{4 p}} .
\end{aligned}
$$

Finally, we note that the expression $\Pi\left((u)_{R / 2}\right)$ is well defined for $0<R \ll 1$ sufficiently small, since the Poincaré inequality implies

$$
\operatorname{dist}\left((u)_{R / 2}, \mathcal{M}\right) \leqq c R\left(f_{B_{2 R}}|D u|^{p}\right)^{\frac{1}{p}} \leqq c R(1+\Lambda)
$$


by the initial assumption. In which case, using the Lipschitz properties of the retraction (cf. Remark 2.3) and $p \geqq 2$, we bound

$$
\begin{aligned}
|\nabla \Pi(u) A-A| \leqq & \left|\nabla \Pi(u)(D u)_{\frac{R}{2}}-\nabla \Pi\left(\Pi\left((u)_{\frac{R}{2}}\right)\right)(D u)_{\frac{R}{2}}\right| \\
& +\left|\nabla \Pi\left(\Pi\left((u)_{\frac{R}{2}}\right)\right)(D u)_{\frac{R}{2}}-(D u)_{\frac{R}{2}}\right| \\
\leqq & c\left|u-(u)_{\frac{R}{2}}\right|+f_{B_{\frac{R}{2}}}\left|\nabla \Pi\left(\Pi\left((u)_{\frac{R}{2}}\right)\right) D u-\nabla \Pi(u) D u\right| \\
\leqq & \left|u-(u)_{\frac{R}{2}}\right|+c\left(f_{B_{\frac{R}{2}}}\left|u-(u)_{\frac{R}{2}}\right|^{\frac{p}{p-1}}\right)^{1-\frac{1}{p}}\left(f_{B_{\frac{R}{2}}}|D u|^{p}\right)^{\frac{1}{p}} \\
\leqq & c\left|u-(u)_{\frac{R}{2}}\right|+c R\left(f_{B_{2 R}}|D u|^{p}\right)^{\frac{2}{p}} .
\end{aligned}
$$

Then by (10), (11b) and (11c) together with the fact that $|V(\xi)|^{2 \alpha} \leqq c\left(1+|\xi|^{q}\right)$, as $1<\alpha<q / p$, we conclude

$$
\left(f_{B_{R}}|V(\nabla \Pi(u) A-A)|^{2 \alpha}\right)^{\frac{1}{2 \alpha}} \leqq c R\left(f_{B_{2 R}} 1+|D u|^{p}\right)^{\frac{q}{2 \alpha p}}+c R\left(f_{B_{2 R}} 1+|D u|^{p}\right)^{\frac{q}{\alpha p}} .
$$

The desired result now follows from (39) together with the initial assumption bounds.

\section{Excess-Improvement and Iteration} result:

We now look to prove Theorem 1.2 via the the following excess-improvement

Proposition 10.1. If for every $\Lambda>0$ and for every $0<\varepsilon<1$, under the assumptions of Theorem 1.2 , the map $u \in W_{\mathrm{loc}}^{1, p}(\Omega, \mathcal{M})$ is an holonomic $\mathfrak{F}$-minimiser such that the smallness conditions

$$
\left|(u)_{x_{0}, R}\right| \leqq \Lambda, \quad\left|(D u)_{x_{0}, R}\right| \leqq \Lambda, \quad \mathcal{E}\left(x_{0}, R\right) \leqq \varepsilon
$$

hold for some $B_{R}\left(x_{0}\right) \subset \subset \Omega$ with $R=R(n, N, p, \Lambda)$ sufficiently small, then for every $0<\tau \leqq \frac{1}{8}$ we have

$$
\mathcal{E}\left(x_{0}, \tau R\right)^{2} \leqq C\left(\tau^{2}+\tau^{-n} \widetilde{\omega}\left(R^{\beta}+\varepsilon\right)\right) \mathcal{E}\left(x_{0}, R\right)^{2}+C \tau^{-n} \widetilde{\omega}\left(R^{\beta}+\varepsilon\right) R^{\beta}
$$

for some $C=C\left(n, N, p, \gamma, \Gamma, \Lambda, K_{\Lambda+1}\right)>0$, some $\beta \in(0,1)$ and a modulus of continuity $\widetilde{\omega}$ independent of $\tau, R$ and $\varepsilon$.

Proof. Firstly, from Lemma 5.1 there exists $q=q(n, N, p, \gamma, \Gamma)>p$ such that $D u \in L_{\text {loc }}^{q}$. Now for fixed $\Lambda>0$ and $0<\varepsilon<1$ assume the initial smallness conditions $\left|(u)_{x_{0}, 4 R}\right| \leqq \Lambda,\left|(D u)_{x_{0}, 4 R}\right| \leqq \Lambda$ and $\mathcal{E}\left(x_{0}, 4 R\right) \leqq \varepsilon$ hold for some 
$B_{4 R}\left(x_{0}\right) \subset \subset \Omega$. Then by (4) and (29) the bilinear form $\mathbb{A}$ given by (30) is bounded and strongly elliptic in the sense of (23). With $\alpha=\frac{2 q}{p+q} \in\left(1, \frac{q}{p}\right)$ let

$$
\widetilde{\omega}(t)=\left(\omega\left(t^{\frac{1}{2}}\right)^{\frac{1}{2}}+t^{\frac{1}{2}}\right)^{\frac{\alpha-1}{2 \alpha-1}},
$$

where $\omega$ is the modulus of continuity given by (5). So for $\varepsilon_{0}=\widetilde{\omega}\left(\varepsilon+R^{\beta}\right)$, with $0<R \ll 1$ sufficiently small and some $0<\beta<1$, the approximate tangential harmonicity of Proposition 8.3 shows the holonomic $\mathfrak{F}$-minimiser $u$ to be a $\delta$ approximate tangential $\mathbb{A}$-harmonic map about $\theta=\Pi\left((u)_{x_{0}, R}\right)$ with $\delta=\delta\left(\varepsilon_{0}\right)=$ $O\left(\varepsilon_{0}{ }^{2+\frac{1}{\alpha-1}}\right)$, since we require $\delta \geqq C\left(\omega(e)^{\frac{1}{2}}+e\right)$ where $e=\left(R^{\beta}+\mathcal{E}(R)^{2}\right)^{\frac{1}{2}}$. Then by Lemma 7.7 there exists a unique tangential $\mathbb{A}$-harmonic map $h \in \mathrm{C}_{\theta}(u)+$ $W_{0}^{1, p}\left(B_{R}, \mathbb{R}^{N}\right)$ about $\theta$ that satisfies

$$
\begin{aligned}
f_{B_{R}}|V(D u-D h)|^{2} \leqq & \varepsilon_{0}\left[R^{\beta}+\mathcal{E}(R)^{2}+\mathcal{E}(R)^{p}\right. \\
& \left.+\left(f_{B_{R}}\left|V\left(D u-(D u)_{R}\right)\right|^{2 \alpha}\right)^{\frac{1}{\alpha}}\right] .
\end{aligned}
$$

Now for any $0<\tau \leqq \frac{1}{2}$ and any matrix $A \in \mathbb{R}^{N n}$ we have $\mathcal{E}\left(x_{0}, \tau R\right)^{2} \leqq c f_{B_{\tau R}}|V(D u-A)|^{2} \leqq c f_{B_{\tau R}}|V(D h-A)|^{2}+|V(D u-D h)|^{2}$. Thus by setting $A=(D h)_{\tau R}$ the interior estimates of Remark 7.4 imply

$$
\begin{aligned}
f_{B_{\tau R}}\left|V\left(D h-(D h)_{x_{0}, \tau R}\right)\right|^{2} & \leqq C \tau^{2} \int_{B_{R}}\left|V\left(D h-(D h)_{x_{0}, R}\right)\right|^{2} \\
& \leqq C \tau^{2} \int_{B_{R}}\left|V\left(D u-(D u)_{x_{0}, R}\right)\right|^{2}
\end{aligned}
$$

since $(D h)_{R}=\nabla \Pi(\theta)(D u)_{R}$ by the divergence theorem and Lemma 7.5 we have $\|D h-\widetilde{A}\|_{p} \leqq C\|\nabla \Pi(\theta) D u-\widetilde{A}\|_{p}$ for any $\widetilde{A} \in \mathbb{R}^{N n}$ with $\nabla \Pi(\theta) \widetilde{A}=\widetilde{A}$ almost everywhere. Moreover, the conclusion of Lemma 7.7 together with Corollary 9.3 implies

$$
\begin{aligned}
f_{B_{\tau R}}|V(D u-D h)|^{2} \leqq & \tau^{-n} f_{B_{R}}|V(D u-D h)|^{2} \\
\leqq & \tau^{-n} \varepsilon_{0}\left[R^{\beta}+\mathcal{E}(R)^{2}+\mathcal{E}(R)^{p}\right. \\
& \left.+\left(f_{B_{R}}\left|V\left(D u-(D u)_{R}\right)\right|^{2 \alpha}\right)^{\frac{1}{\alpha}}\right] \\
\leqq & C \tau^{-n} \varepsilon_{0}\left(R^{\beta}+\mathcal{E}\left(x_{0}, R\right)^{2}+\mathcal{E}\left(x_{0}, R\right)^{p}+\mathcal{E}\left(x_{0}, 2 R\right)^{2}\right)
\end{aligned}
$$

Thus $\mathcal{E}(\tau R)^{2} \leqq C\left(\tau^{2}+\tau^{-n} \varepsilon_{0}\right) \mathcal{E}(2 R)^{2}+C \tau^{-n} \varepsilon_{0} R^{\beta}$ for every $0<\tau \leqq 1 / 2$. 
Given Proposition 10.1 one can judiciously choose the parameter $\tau$ such that both

$$
C \tau^{2} \leqq \frac{1}{2} \tau^{\beta / 2} \text { and } C \tau^{-n} \widetilde{\omega}\left(R^{\beta}+\varepsilon\right) \leqq \frac{1}{2} \tau^{\beta / 2}
$$

hold. As the first inequality is equivalent to choosing $0<\tau \leqq(2 C)^{-1 /(2-\beta / 2)}=\tau_{0}$ we must also require $0<\widetilde{\omega}\left(R^{\beta}+\varepsilon\right) \leqq \frac{1}{2 C} \tau^{n+2}$. Then for such (fixed) $\tau$ we can decrease $\varepsilon$ and $R$ even further, if necessary, to obtain:

Corollary 10.2. For every $\Lambda>0$ there exists $\tau=\tau(\Lambda)$ and $\varepsilon=\varepsilon(\Lambda)$ such that if the smallness conditions

$$
\left|(u)_{x_{0}, R}\right| \leqq \Lambda, \quad\left|(D u)_{x_{0}, R}\right| \leqq \Lambda, \quad \mathcal{E}\left(x_{0}, R\right) \leqq \varepsilon
$$

hold on some $B_{R}\left(x_{0}\right) \subset \subset \Omega$ with $0<R \ll 1$ sufficiently small, then

$$
\mathcal{E}\left(x_{0}, \tau R\right)^{2} \leqq \tau^{\beta / 2} \mathcal{E}\left(x_{0}, R\right)^{2}+R^{\beta} .
$$

By iterating this result we get:

Claim 10.3. For every $\Lambda>0$ there exists $\varepsilon=\varepsilon(\Lambda+1)$ such that for integers $j \geqq 1$ we have

$$
\mathcal{E}\left(x_{0}, \tau^{j} R\right)^{2} \leqq \tau^{j \beta / 2} \mathcal{E}\left(x_{0}, R\right)^{2}+R^{\beta} \frac{\tau^{j \beta / 2}-\tau^{j \beta}}{\tau^{\beta / 2}-\tau^{\beta}},
$$

whenever $\left|(u)_{x_{0}, R}\right| \leqq \Lambda,\left|(D u)_{x_{0}, R}\right| \leqq \Lambda, \mathcal{E}\left(x_{0}, R\right) \leqq \varepsilon$ holds on some $B_{R}\left(x_{0}\right) \subset \subset \Omega$ with $0<R \ll 1$ sufficiently small.

Now if $r \in[\tau R, R)$ then $\mathcal{E}\left(x_{0}, r\right)^{2} \leqq \tau^{-n} \mathcal{E}\left(x_{0}, R\right)^{2}$; whereas if $r \in(0, \tau R]$ there exists an integer $j \geqq 1$ such that $\tau^{\overline{j+1}} R \leqq r \leqq \tau^{j} R$ from which Claim 10.3 implies

$$
\mathcal{E}\left(x_{0}, r\right)^{2} \leqq \tau^{-n} \mathcal{E}\left(x_{0}, \tau^{j} R\right)^{2} \leqq\left(\frac{r}{R}\right)^{\beta / 2} \tau^{-n-\beta / 2}\left[\mathcal{E}\left(x_{0}, R\right)^{2}+\frac{R^{\beta}}{\tau^{\beta / 2}-\tau^{\beta}}\right] .
$$

Thus for every $x_{0} \in \operatorname{Reg} u$ there exists $\Lambda>0$, an $\varepsilon>0$ sufficiently small and an $0<R<\operatorname{dist}\left(x_{0}, \partial \Omega\right)$ sufficiently small such that

$$
\mathcal{E}\left(x_{0}, r\right)^{2} \leqq\left(\frac{r}{R}\right)^{\beta / 2} \tau^{-n-\beta / 2}\left[\varepsilon^{2}+\frac{R^{\beta}}{\tau^{\beta / 2}-\tau^{\beta}}\right], \quad 0<r<R,
$$

whenever $\left|(u)_{x_{0}, R}\right| \leqq \Lambda,\left|(D u)_{x_{0}, R}\right| \leqq \Lambda$ and $\mathcal{E}\left(x_{0}, R\right) \leqq \varepsilon$ hold (which by Remark 1.6 is what one seeks to demonstrate).

Acknowledgments. The author wishes thank JAN KRISTENSEN for numerous discussions and suggestions throughout the development of this paper. Further thanks are extended to the anonymous reviewers, for their careful reading of the manuscript and their many insightful comments and suggestions, as well as HEMANTH SARATCHANDRAN for his advice and useful discussions on homotopy theory. The support of the Oxford Centre for Nonlinear PDE (OxPDE) through the EPSRC Science and Innovation award to OxPDE (EP/E035027/1) is also gratefully acknowledged. 
Open Access This article is distributed under the terms of the Creative Commons Attribution 4.0 International License (http://creativecommons.org/licenses/by/4.0/), which permits unrestricted use, distribution, and reproduction in any medium, provided you give appropriate credit to the original author(s) and the source, provide a link to the Creative Commons license, and indicate if changes were made.

\section{References}

1. Ambegaokar, V., De Gennes, P.G., Rainer, D.: Landau-Ginsburg equations for an anisotropic superfluid. Phys. Rev. A 9, 2676-2685 (1974)

2. Acerbi, E., Fusco, N.: Semicontinuity problems in the calculus of variations. Arch. Ration. Mech. Anal. 86(2), 125-145 (1984)

3. Acerbi, E., Fusco, N.: A regularity theorem for minimizers of quasiconvex integrals. Arch. Ration. Mech. Anal. 99(3), 261-281 (1987)

4. Acerbi, E., Fusco, N.: An approximation lemma for $W^{1, p}$ functions. Material Instabilities in Continuum Mechanics (Edinburgh, 1985-1986), 1-5, 1988

5. Acerbi, E., Fusco, N.: Local regularity for minimizers of non convex integrals. Ann. Scuola Norm. Sup. Pisa Cl. Sci. (4) 16(4), 603-636 (1989)

6. BECK, L.: Boundary regularity results for variational integrals. Q. J. Math. 62(4), 791824 (2011)

7. Bethuel, F.: A new obstruction to the extension problem for Sobolev maps between manifolds. J. Fixed Point Theory Appl. 15(1), 155-183 (2014)

8. Botт, R.: On torsion in Lie groups. Proc. Natl. Acad. Sci. USA 40, 586-588 (1954)

9. Béthuel, F., Chiron, D.: Some questions related to the lifting problem in Sobolev spaces. Perspectives in Nonlinear Partial Differential Equations, 125-152, 2007

10. Béthuel, F., Demengel, F.: Extensions for Sobolev mappings between manifolds. Calc. Var. Partial Differ. Equ. 3(4), 475-491 (1995)

11. Berreman, D.W., Meiboom, S.: Tensor representation of Oseen-Frank strain energy in uniaxial cholesterics. Phys. Rev. A 30, 1955-1959 (1984)

12. Bousquet, P., Ponce, A.C., Van Schaftingen, J.: Density of smooth maps for fractional Sobolev spaces $W^{s, p}$ into $\ell$ simply connected manifolds when $s \geqslant 1$. Conflu. Math. 5(2), 3-22 (2013)

13. Bousquet, P., Ponce, A.C., Van Schaftingen, J.: Strong approximation of fractional Sobolev maps. J. Fixed Point Theory Appl. 15(1), 133-153 (2014)

14. Bröcker, T., tOM Dieck, T.: Representations of compact Lie groups. Graduate Texts in Mathematics, Vol. 98. Springer, New York, 1985

15. Bruder, C., Vollhardt, D.: Symmetry and stationary points of a free energy: the case of superfluid ${ }^{3}$ He. Phys. Rev. B 34, 131-146 (1986)

16. Campanato, S.: Proprietà di hölderianità di alcune classi di funzioni. Ann. Scuola Norm. Sup. Pisa (3) 17(1-2), 175-188 (1963)

17. Carozza, M., Fusco, N., Mingione, G.: Partial regularity of minimizers of quasiconvex integrals with subquadratic growth. Ann. Mat. Pura Appl. (4) 175, 141-164 (1998)

18. Campanato, S., Stampacchia, G.: Sulle maggiorazioni in $L^{p}$ nella teoria delle equazioni ellittiche. Boll. Un. Mat. Ital. (3) 20, 393-399 (1965)

19. Deutsch, F.: The convexity of Chebyshev sets in Hilbert space. Topics in Polynomials of One and Several Variables and Their Applications, 143-150, 1993

20. De Gennes, P.G.: Short range order effects in the isotropic phase of nematics and cholesterics. Mol. Cryst. Liq. Cryst. 12(3), 193-214 (1971)

21. De Gennes, P.G.: Long range distortions in an anisotropic superfluid. Phys. Lett. A 44(4), 271-272 (1973)

22. De Giongi, E.: Frontiere orientate di misura minima. Seminario di Matematica della Scuola Normale Superiore di Pisa, 1960-61. Editrice Tecnico Scientifica, Pisa, 1961 
23. De Giongi, E.: Selected Papers (Eds. Ambrosio, L., Maso, G.D., Forti, M., Miranda, M. and Spagnolo, S.) Springer, Berlin, 2006

24. Duzana, F., Gastel, A., Grotowski, J.F.: Partial regularity for almost minimizers of quasi-convex integrals. SIAM J. Math. Anal. 32(3), 665-687 (2000)

25. Duzane, F., Grotowski, J.F., Kronz, M.: Regularity of almost minimizers of quasiconvex variational integrals with subquadratic growth. Ann. Mat. Pura Appl. (4) 184(4), 421-448 (2005)

26. Dejarnette, N., Hajasz, P., Lukyanenko, A., Tyson, J.T.: On the lack of density of Lipschitz mappings in Sobolev spaces with Heisenberg target. Conform. Geom. Dyn. 18, 119-156 (2014)

27. Diening, L., Lengeler, D., Stroffolini, B., Verde, A.: Partial regularity for minimizers of quasi-convex functionals with general growth, SIAM J. Math. Anal. 44(5), 3594-3616 (2012)

28. Duzana, F., Mingione, G.: The p-harmonic approximation and the regularity of pharmonic maps, Calc. Var. Partial Differ. Equ. 20(3), 235-256 (2004)

29. Duzana, F., Mingione, G.: Harmonic type approximation lemmas. J. Math. Anal. Appl. 352(1), 301-335 (2009)

30. Diening, L. Stroffolini, B., Verde, A.: The $\varphi$-harmonic approximation and the regularity of $\varphi$-harmonic maps. J. Differ. Equ. 253(7), 1943-1958 (2012)

31. Ekeland, I.: Nonconvex minimization problems. Bull. Am. Math. Soc. (N.S.) 1(3), 443-474 (1979)

32. Evans, L.C.: Quasiconvexity and partial regularity in the calculus of variations. Arch. Ration. Mech. Anal. 95(3), 227-252 (1986)

33. Evans, L.C., GariePY, R.F.: Blowup, compactness and partial regularity in the calculus of variations. Indiana Univ. Math. J. 36(2), 361-371 (1987)

34. Evans, L.C., Gariepy, R.F.: Partial regularity for constrained minimizers of convex or quasiconvex functionals. Rend. Sem. Mat. Univ. Politec. Torino, Vol. 47, 1989. [Special issue, 75-93. Some topics in nonlinear PDEs (Turin, 1989)]

35. Evans, L.C., GARIEPY, R.F.: Measure Theory and Fine Properties of Functions, Studies in Advanced Mathematics. CRC Press, Boca Raton, 1992

36. Federer, H.: Curvature measures. Trans. Am. Math. Soc. 93, 418-491 (1959)

37. Federer, H., Fleming, W.H.: Normal and integral currents. Ann. Math. (2) 72, 458-520 (1960)

38. Foote, R.L.: Regularity of the distance function. Proc. Am. Math. Soc. 92(1), 153-155 (1984)

39. Fusco, N., Hutchinson, J.: $C^{1, \alpha}$ partial regularity of functions minimising quasiconvex integrals. Manuscr. Math. 54(1-2), 121-143 (1985)

40. Giaquinta, M., Giusti, E.: On the regularity of the minima of variational integrals. Acta Math. 148, 31-46 (1982)

41. Giaquinta, M., Giusti, E.: The singular set of the minima of certain quadratic functionals. Ann. Scuola Norm. Sup. Pisa Cl. Sci. (4) 11(1), 45-55 (1984)

42. GIaQuinta, M.: Multiple integrals in the calculus of variations and nonlinear elliptic systems. Annals of Mathematics Studies, Vol. 105. Princeton University Press, Princeton, 1983

43. Gallot, S., Hulin, D., Lafontaine, J.: Riemannian geometry. Universitext, 3rd edn. Springer, Berlin, 2004

44. Giaquinta, M., Martinazzi, L.: An introduction to the regularity theory for elliptic systems, harmonic maps and minimal graphs. Appunti. Scuola Normale Superiore di Pisa (Nuova Serie), 2nd edn., Vol. 11. Edizioni della Normale, Pisa, 2012

45. Giaquinta, M., Modica, G.: Regularity results for some classes of higher order nonlinear elliptic systems, J. Reine Angew. Math. 311/312, 145-169 (1979)

46. Giaquinta, M., Modica, G.: Partial regularity of minimizers of quasiconvex integrals. Ann. Inst. H. Poincaré Anal. Non Linéaire 3(3), 185-208 (1986)

47. Gilbarg, D., Trudinger, N.S.: Elliptic partial differential equations of second order. Classics in Mathematics. Springer, Berlin, 2001. (Reprint of the 1998 edition) 
48. Hang, F.: On the weak limits of smooth maps for the Dirichlet energy between manifolds. Commun. Anal. Geom. 13(5), 929-938 (2005)

49. Hatcher, A.: Algebraic Topology. Cambridge University Press, Cambridge, 2002

50. HÉLein, F.: Harmonic maps, conservation laws and moving frames. Cambridge Tracts in Mathematics, 2nd edn., Vol. 150. Cambridge University Press, Cambridge, 2002

51. Hertz, H.: Gesammelte Werke. Band III: Die Prinzipien der Mechanik in neuem Zusammenhange dargestellt. Johann Ambrosius Barth Verlag, Leipzig, 1894

52. Hu, S.-T.: Homotopy theory. Pure and Applied Mathematics, Vol. 8. Academic Press, New York, 1959

53. Hardt, R., Kinderlehrer, D., Lin, F.-H.: Stable defects of minimizers of constrained variational principles. Ann. Inst. H. Poincaré Anal. Non Linéaire 5(4), 297-322 (1988)

54. Hildebrandt, S., Kaul, H., Widman, K.-O.: An existence theorem for harmonic mappings of Riemannian manifolds. Acta Math. 138(1-2), 1-16 (1977)

55. Hang, F., Lin, F.: Topology of Sobolev mappings. II. Acta Math. 191(1), 55-107 (2003)

56. Hardt, R., Lin, F.-H.: Mappings minimizing the $L^{p}$ norm of the gradient. Commun. Pure Appl. Math. 40(5), 555-588 (1987)

57. Hofmann, K.H., Morris, S.A.: The structure of compact groups: a primer for the student-a handbook for the expert. de Gruyter Studies in Mathematics, 2nd revised and augmented edn., Vol. 25. Walter de Gruyter \& Co., Berlin, 2006

58. Hildebrandt, S., Widman, K.-O.: Some regularity results for quasilinear elliptic systems of second order. Math. Z. 142, 67-86 (1975)

59. Kristensen, J., Mingione, G.: The singular set of Lipschitzian minima of multiple integrals. Arch. Ration. Mech. Anal. 184(2), 341-369 (2007)

60. Kristensen, J., TAheri, A.: Partial regularity of strong local minimizers in the multidimensional calculus of variations. Arch. Ration. Mech. Anal. 170(1), 63-89 (2003)

61. LeE, J.M.: Introduction to smooth manifolds. Graduate Texts in Mathematics, 2nd ed., Vol. 218. Springer, New York, 2013

62. Leggett, A.J.: A theoretical description of the new phases of liquid ${ }^{3} \mathrm{He}$. Rev. Mod. Phys. 47, 331-414 (1975)

63. Luckhaus, S.: Partial Hölder continuity for minima of certain energies among maps into a Riemannian manifold. Indiana Univ. Math. J. 37(2), 349-367 (1988)

64. Luckhaus, S.: Convergence of minimizers for the p-Dirichlet integral. Math. Z. 213(3), 449-456 (1993)

65. Longa, L., Monselesan, D., Trebin, H.-R.: An extension of the Landau-Ginzburgde Gennes theory for liquid crystals. Liq. Cryst. 2(6), 769-796 (1987)

66. Longa, L., Trebin, H.-R.: Structure of the elastic free energy for chiral nematic liquid crystals. Phys. Rev. A 39, 2160-2168 (1989)

67. Mermin, N.D.: The topological theory of defects in ordered media. Rev. Mod. Phys. 51, 591-648 (1979)

68. Milnor, J.: Morse theory. Annals of Mathematics Studies. Princeton University Press, Princeton, 1963

69. Mingione, G.: Regularity of minima: an invitation to the dark side of the calculus of variations. Appl. Math. 51(4), 355-426 (2006)

70. Morrey, C.B. Jr.: The problem of Plateau on a Riemannian manifold. Ann. Math. (2) 49, 807-851 (1948)

71. Morrey, C.B. Jr.: Quasi-convexity and the lower semicontinuity of multiple integrals. Pac. J. Math. 2, 25-53 (1952)

72. Munkres, J.R.: Elementary differential topology. Annals of Mathematics Studies. Princeton University Press, Princeton, 1963

73. Mucci, D., Nicolodi, L.: On the elastic energy density of constrained Q-tensor models for biaxial nematics. Arch. Ration. Mech. Anal. 206(3), 853-884 (2012)

74. Mermin, N.D., Stare, C.: Ginzburg-Landau approach to $L \neq 0$ pairing. Phys. Rev. Lett. 30, 1135-1138 (1973)

75. Osheroff, D.D., Gully, W.J., Richardson, R.C., Lee, D.M.: New magnetic phenomena in liquid $\mathrm{He}^{3}$ below 3 mK. Phys. Rev. Lett. 29, 920-923 (1972) 
76. Osheroff, D.D., Richardson, R.C., LeE, D.M.: Evidence for a new phase of solid $\mathrm{He}^{3}$, Phys. Rev. Lett. 28, 885-888 (1972)

77. Riesz, M.: L'intégrale de Riemann-Liouville et le problème de Cauchy. Acta Math. 81, $1-223(1949)$

78. SimON, L.: Theorems on regularity and singularity of energy minimizing maps. Lectures in Mathematics ETH Zürich. Birkhäuser, Basel, 1996

79. Schoen, R., Uhlenbeck, K.: A regularity theory for harmonic maps. J. Differ. Geom. 17(2), 307-335 (1982)

80. SalomaA, M.M., Volovik, G.E.: Quantized vortices in superfluid ${ }^{3}$ He. Rev. Mod. Phys. 59, 533-613 (1987)

81. Vissenberg, M.C.J.M., Stallinga, S., Vertogen, G.: Generalized Landau-de Gennes theory of uniaxial and biaxial nematic liquid crystals. Phys. Rev. E 55, 4367-4377 (1997)

82. Vollhardt, D., Wölfle, P.: The Superfluid Phases of Helium 3, Dover Books on Physics. Dover Publications, Mineola, 2013. (Reprint of the 1990 edition)

83. White, B.: Infima of energy functionals in homotopy classes of mappings. J. Differ. Geom. 23(2), 127-142 (1986)

84. White, B.: Homotopy classes in Sobolev spaces and the existence of energy minimizing maps. Acta Math. 160(1-2), 1-17 (1988)

85. Whitehead, J.H.C.: On $C^{1}$-complexes. Ann. Math. (2) 41(4), 809-824 (1940)

The Queen's College, University of Oxford,

Oxford,

UK.

e-mail: hopper@maths.ox.ac.uk

(Received February 27, 2015 / Accepted March 5, 2016)

Published online May 2, 2016 - (C) The Author(s) (2016)

This article is published with open access at Springerlink.com 